\title{
Anhang 1 \\ Übersicht aufgenommener Handschriften
}

Matthias H. O. Schulz 

In die kritische Edition wurden 172 sahidische Handschriften aufgenommen, davon 5 vollständig (1, 4, 5, 9 und 19) und 5 überwiegend vollständig (10, 103, 106, 110 und 116). Der Rest ist fragmentiert bis stark fragmentiert, doch nur die Handschrift 47 musste wegen $\mathrm{zu}$ schlechter Lesbarkeit ausgesondert werden. Handschriften sind nach der Nummerierung der SMR-Liste (Schmitz-MinkRichter-Liste) des Instituts für Neutestamentliche Textforschung der Westfälischen Wilhelms-Universität zu Münster bezeichnet, der in Koptologie und neutestamentlicher Wissenschaft maßgeblichen Referenzliste koptischer neutestamentlicher Handschriften. Für drei Fragmente lag bei Drucklegung kein paläographischer Abgleich mit nummerierten Handschriften durch S. G. Richter und K. D. Schröder vor; sie sind nach Bibliothekssignaturen bzw. Katalognummern angeführt. Drei weitere Kodizes sind Neufunde aus Deir el-Bachit. Vorangestellt sind die Handschriften in Lykopolitanisch und Proto-Bohairisch.

Um einen leicht zugänglichen Überblick zu Handschriften und ihren Versen aus dem Johannesevangelium zu erlauben, werden nur notwendigste Informationen angeführt. Handschriftensigel verweisen auf die SMR-Datenbank, durch die sich weiterführende Literatur ermitteln lässt und die eine Konkordanz zur nun obsoleten Nummerierung in Schüsslers Biblia Coptica bietet. Nicht für jede Handschrift sind alle Angaben angeführt oder notwendig. Aufbau der Einträge:

Nummer (fallweise Angabe der Art der Handschrift) || Datierung || Herkunft || Material, Folien/Fragmentanzahl || Kolumnen || Zeilen || Inhalt (Verse aus J, andere Inhalte optional und nicht vollständig) || (besondere Bemerkungen) || (Paratextelemente)

Für die Auflistung, doch auch für Angaben zu Paratexten, die der Handschriftenliste folgen, werden folgende Abkürzungen verwendet:

\begin{tabular}{|c|c|c|c|}
\hline$*$ & ursprünglich & $\rightarrow$ & markiert durch \\
\hline$\odot / \odot \odot$ & Koronis / Koroniden & $\dot{x}$ & Ornament / ornamentiert \\
\hline , / & Diple / Diplen & पा / बाषा & Paragraphos / Paragraphoi \\
\hline oo & Kreise & $\div 1 \div \div$ & Obelos / Obeloi \\
\hline$\stackrel{\star}{\star}$ & Abschlussmarkierung & $\neq$ & Perikopenmarkierung \\
\hline ca. & circa & can. & Eusebianische Kanon- und Abschnitts-Nr(n). \\
\hline Fol. & Folio / Folien & Fragm. & Fragment $(\mathrm{e})($ fragm. $=$ fragmentarisch $)$ \\
\hline Ini. & Initiale(n) & Jh. & Jahrhundert \\
\hline Keph. & Kephalaion- $\mathrm{Nr}(\mathrm{n})$. & Kol. & Kolumne / Kolumnen \\
\hline & verso / Verso & & vergrößert \\
\hline Zit. & Zitat & & \\
\hline
\end{tabular}

In die Auflistung wurde 47 zwar aufgenommen, doch in geschweifte Klammern gesetzt; Paratexte der Handschrift wurden den ergänzenden Materialien zur Edition beigegeben. 
Ein von Horner nachkollationiertes Fragment (Paris, Lou, E 9967 [Revillout 126]) ließ sich nicht verifizieren, zitiert aber ohnehin nur den Beginn von J $13 .^{70}$ Nach Horner habe es als Textvarianten die Auslassung von понне м̄сммюм пискаpıOTHC in 13,2 und die Schreibung N̄̄̄ka in 13,3.

Die angeführten Verse der Handschriftenliste und der folgenden Verzeichnung der Einzelversbezeugung spiegeln wider, was die Vollkollationen als Texterhaltung für jede Einzelhandschrift ergaben; die Angaben entsprechen im Detail nicht immer dem, was SMR-Liste oder BC verzeichnen, sondern präzisieren und korrigieren dort gebotene Angaben. Für pbo, ly 1 und ly 2 werden erstmals genaue Angaben geliefert, welche Verse die Handschriften überliefern.

Da es sich bis auf pbo, ly 1 und ly 2 um sahidische Handschriften handelt und alle Manuskripte durch SMR-Nummern identifizierbar sind, unterbleibt wie beim kritischen Apparat der Edition im Folgenden die Angabe des Kürzels „sa“ vor Handschriftennummern und in anderen Kontexten.

ly 1 4. Jh. (Thompson) || Herkunft unbekannt || Papyrus, 43 Fol. (teils fragm.) || 1 Kol. || 33-37 Zeilen || 2,12-3,21; 4,*5.6-5,3.5-7,10.*11.28.30-52; 8,12-12,44.46-13,14.16-17,22. $24-19,41 ; 20, * 3.4-7 . * 8.13-27$

ly 2 Textauszug (,literarischer Text in nicht-literarischem Dokument“ Funk/Smith) ॥ 300-350 A.D. (Chester Beatty Library) || Herkunft unbekannt || Papyrus, Fol. || 1 Kol. || 18-37 Zeilen $(* 28-37)$ || 10,*7.8-12,44.46-13,12.14.*16.17-18.*22.23-26. *27. *28. *29. 31.32.*33.35-38 (Angabe nach der Edition)

pbo 4. Jh. (Kasser) || wohl Dishna [„Dishna Papers“] (Robinson) || Papyrus, ca. 74 Fol. und mehrere Fragm. von Fol. (*?) || 1 Kol. || 18-26 Zeilen || 1,1-2.9.*10.*14.*15.*16.*17.*18. $* 19 . * 20 . * 21 . * 22 . * 23 . * 24 . * 25 . * 40 . * 45 ; 2, * 9 . * 11 . * 15 . * 16 ; * 3,33 ; 4, * 5 . * 6 . * 13.14 . * 15.20-54$; 5,1-3.5-7,52; 8,12- 21,25 || |ा|, Subscriptio

1 1. Hälfte 5. Jh. (Quecke), 5. Jh. (Orlandi) || Herkunft unbekannt || Pergament, 230 Fol. || 2 Kol. || 23 Zeilen || 1,1-5,3.5-7,52; 8,12-9,37.39-16,14.16-21,25 || थाा, Ini., Zit. $\rightarrow$ », Subscriptio

3 5. Jh. (Luft), 6. Jh. (Beltz, Crum), 6.-8. Jh. (Schubart) || Herkunft unbekannt || Pergament, 18 Fol. || 1 Kol. || 17-21 Zeilen || 1,1-3,2; 3,10-36; 4,1-29.39-47; 5,36-47; 6,1-9 || पापा

4 ca. 600 (Thompson, Buchthal/Kurz), 9. Jh. ? (Kasser) || Jeremiaskloster, Saqqara \| Pergament, 201 Fol. || 1 Kol. || 29 Zeilen || 1,1-5,3.5-7,52; 8,12-21,25 || शाभा, Ini., Zit. $\rightarrow$ », „Amen-Worte“ nummeriert (1-25), Subscriptio, Kolophon

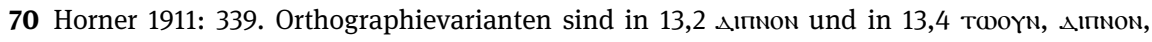
готе. 
Miniatur-Kodex || 6. Jh. (Hintze/Schenke), ca. 600 (Buchthal/Kurz), 7. Jh. (Thompson); 9. Jh. ? (Kasser) || Jeremiaskloster, Sakkara || Pergament, 168 Fol. || 1 Kol. || 19- 28 Zeilen || 1,1-5,3.5- 7,52; 8,12-21,25 || बाभा, Ini. (wenige), Zit. $\rightarrow$ », Subscriptio, Kolophon

4. Jh. (Horner), 7. Jh. (Layton) || Theben (Crum) || Papyrus, 25 Fol. (fragm.) || 1 Kol. || 7 23 Zeilen (*ca. 25) || 4,35-36.39-40; 6,70-71; 7,1.3-5; 12,6-8.12-13.16-18.21-23.2627.29-32.35.38-40.42-45.47-49; 13,1-2.5-7.10-11.14-16.19-20.*21.23-25.28-30.33-34; $15,20-24.25-27 ; 16,1-7.8-17.18-20 ; 20,8-11.13-15$

4. Jh. (Horner), 7. Jh. (Layton) || Herkunft unbekannt || Papyrus, 14 Fol. (fragm.) || 1 Kol. || 4-17 Zeilen $(* 17)$ || 3,*32.33-35.36; 4,1.50.*52.53; 6,38-57.*58.64-69; 10,35-40; $13, * 33 . * 34.36 ; 15, * 14.15-17.18-22 ; 17,21-22 . * 23 . * 24 ; 18,6-10.11-15$

7./8. Jh. ? (Petersen), 8. Jh. (Schenke), 8.-9. Jh. (Cramer), 9. Jh. (van Lantschoot), 9.10. Jh. (Kasser) || Michaels-Kloster, Harabt Hamuli || Pergament, 113 Fol. (*127) || 2 Kol. || 36-39 Zeilen || 1,1-5,3.5-7,52; 8,12-21,25 || Inscriptio, Фाणा, Ini., Keph. (Nr. 8 und 12 am Text unbezeichnet, wohl bei 6,15 und 19,28), Glosse, Subscriptio

578 Anno Martyrum (Kolophon; julianisch: 29.08.861-28.08.862) || Michaels-Kloster, Harabt Hamuli || Pergament, 49 Fol. || 2 Kol. || 33 Zeilen || 1,1.3-35.*36.37-40.*41.422,3.5 - 4,3.*4.5 - 42.*43.44 - 5,2.*3.5 - 21.*22.23 - 39.*40.41 - 6,13.*14.15- 19.21-32.*33. $34-7,5 . * 6.7-20 . * 21.22-24 . * 25.26-41 . * 42.43-52 ; 8,12-44.46-49.51-57 . * 58.59-9,13$. $15-10,1 . * 2.3-6 . * 7.8-29 . * 30 . * 31.32-39.41-11,18 . * 19.20-25 . * 26.27-34.36-44 . * 45.46-$ $12,27 . * 28.29-36 . * 37.38-13,27 . * 28.29-15,11.13-19 . * 20.21-24 . * 25.26-16,13.15-17 . * 18.19$ $-17,16.19-21.23-18,6.8-22 . * 23.25-38 . * 39.40-19,14.16-22 . * 23.24-25 . * 27.28-29.31-34$. *35.36-42; 20,1-22.24-21,2.*3.4-9.*11.12-14.*15.16-19.21-23.25 || Inscriptio, Ini. (vgr. und $\aleph_{\text {) }}$, Abschnitts-Nrn. (vielleicht von m2), $\$$ (I mit Punkten und Doppelkreuz, 19,13-22), Zit. $\rightarrow$ », Keph. (rezent, mit Bleistift geschrieben, im Faksimile nicht erkennbar), Kolophon

5.-6. Jh. (Bethge) || Herkunft unbekannt || Papyrus, 110 Fragm. (ca. 60 identifiziert) || 2 Kol. || ca. *30 Zeilen || 1,8-9.12-14.22.23.26.27.33.*34.*35.36.39-40.42.*43.*44.45.46. *47.48.49.*50.51; 2,1.5.6.9.10.12.15.16.18.19.22.23; 3,1.2.4.6-8.10.11.13.14.16-17.19.20. 23.24.26.27.29.32.36; 4,1.5.6.9-12.14.15.19-20.22.24-25.28.29.33.34.36.37.39.40.43.4445.46-47.50-51.54; 5,1.5.6.9.13.16-18.19.22.24-25.27-28.30.34-35.36-37.39; 8,58.59; $9,1.4 .5 .8 .11-12 . * 16 . * 18 . * 19.20 .22 .25 .28 .29 .33 ; 10,16.19-21.33-36 ; 11,4-5 . * 6.30-31$. *41-42.45.46.48-49.*52.54-55.57; 12,1.2-4.8-9.12.13.16.17.24-25.28.29.32.34.36.40. 42-44; 13,37.38; 14,*3.*7.10.11.13-15.23-24.27; 15,5.6.9.10.19 || Papyrus-Makulatur (teils dokumentarische Texte, wohl zur Reparatur) - Or. 14149(13): zwei Fragm. (Blattunterteile) mit Makulatur; Or. 14149(23) ${ }^{v}$ : ein Fragm. (Blattunterteil) mit Makulatur; Or. 14149(24): zwei Fragm. (mit 1,14 und 1,22-23.26-27) mit Makulatur, Fragm. 11 (mit 1,2223.26-27) wohl antik repariert (Makulatur schräg an die Seite gesetzt, auf $\mathrm{r}$ und v sichtbar, dokumentarischer Text) || Фा|ा, Ini., Abschnitts-Nrn.

6. Jh. (de Ricci, Boud'hors, Sänger-Böhm), 8.-9. Jh. (Schüssler) || Weißes Kloster, Sohag (Schüssler) || Papyrus, 31 Fol. (fragm. Doppelblatthälften) || 1 Kol. || 17-19 Zeilen || $1,1-33 . * 34.35-44 . * 45.45-51 ; 2,1-10 . * 20.21-22.25 ; 3,1-3 . * 4.5-6.8 .26-36 ; 4,1-7 . * 8.15-$ $24.52-54 ; 5,1-3.6-7.17-23.30-40 . * 41.42-47 ; 6,11-27.35-43.52-56.54-58^{\text {sic }} .59 . * 68$. 69-71; 7,1-15.49-52; 8,12-14.15-23.*25.26-29.*30.31-*32.32-41.42-47.*48.49-52. *53.54-59; 9,1-4.7-8.11-14.16.19-20 || Am Unterrand von f. *20r [= Copte 164 II f. 
$16 \mathrm{~m}^{\mathrm{r}}$; 4,52-5,2] sind um $90^{\circ}$ im Uhrzeigersinn zum Text gedreht Reste von 5 Zeilen eines griechischen protokollon (Produktionsvermerk) in einer Kursive wohl der 1. Hälfte des 5. Jh. erkennbar, cf. Sänger-Böhm 2019: 117-123. || || bei 8,40

$14^{\mathrm{L}} \quad$ bilingues Evangelistar (griechisch-sahidisch) || 7./8. und 9./10. Jh. (Heer in verschiedenen Publikationen), 8. Jh. (van Haelst, Kasser), 8. und 9. Jh. (Aland in verschiedenen Publikationen), 8. und 9./10. Jh. (Clark für verschiedene Teile der Handschrift), 11. Jh. (Hedley) || Weißes Kloster, Sohag (Heer, Schüssler), Harabt Hamuli (fälschlich Brakmann) || Pergament, 88 Fol. (*112) || 2 Kol. || 28 Zeilen || 1,10-11.*12.*13.14.35-51; 2,111; 3,1-11; 10,9-18.11-18; 12,12-28; 14,*31; 15,1-16.26-*27; 16,1-7.*8.9-15; 17,4-17; 19,19-20.22-23.*25.*26.27; 20,1-18.24-31 || Rubriken

15L Lektionar || 1. Hälfte 9. Jh. (nach Kolophonen), 9. Jh. (Hyvernat) || Michaels-Kloster, Harabt Hamuli || Pergament, 79 Fol. (+ 1 Fol. einer anderen Hs. als Vorsatzblatt) || 2 Kol. || 28-33 Zeilen || 1,1-14.43-51; 2,1-11; 3,1-15; 5,21-29; 6,5-14; 7,14-24; 10,7.11-18; 12,12-19.20-32; 15,7-17.26-16,13; 17,1-11; 19,25-37; 20,1-18.19-31 || Rubriken

$16^{\mathrm{L}} \quad$ bilingues Pascha-Lektionar (sahidisch-arabisch) || 12./13. Jh. (Hyvernat), nicht vor 13. Jh. (Horner), 13./14. Jh. (Balestri), Ende 14. Jh. (Ciasca), um 1400 (Rahlfs) || Weißes Kloster, Sohag (Schüssler) || Papier, 189 Fol. || 2 Kol. (Rubriken und nichtbiblische Texte teils 1 Kol.) || 26-28 Zeilen || 6,27-58; 8,12-20.21-28.51-59; 10,14-21.23-28.29-42; $11,46-53.55-57 ; 12,1-8.35-43 ; 13,1-20.21-30.33-14,25.26-15,25.26-16,33 ; 17,1-26$; 18,1-2.3-9.10-14.15-27.28-40; 19,1-12.13-27.28-30.31-37.38-42 || Rubriken

493 Anno Hijra (Kolophon; julianisch: 17.11.1099-06.11.1100) || Deir el-Naqlun, Faijum || Papier, 72 Fol. || 1 Kol. || 23-26 Zeilen || 1,1-5,3, 5-7,52; 8,12-10,41.43-12,9.11-44.4621,25 || Inscriptio, Ini., Kolophone (drei, einer arabisch)

22 Exzerpte || 8. Jh. (Bartina) || Herkunft unbekannt || Papyrus, 1 Fragm. || 1 Kol. (*2?) || noch 6 Zeilen (*?) || 17,8.22; 21,16-17

40 9./10. Jh. (Schüssler) || Weißes Kloster, Sohag (Hyvernat) || Pergament, 7 Fol. (fragm.) || 2 Kol. || *45 Zeilen || 1,1-7.17-24.*25.31-41.*42.45-2,1 || Inscriptio, Ini.

\{47 9./10. Jh. (Schüssler) || Herkunft unbekannt || Pergament, 5 Fol. (fragm., dabei 1 Doppelblatt) + 1 Fragm. || 2 Kol. || ca. 27-29 Zeilen || 1,1-2,23 || Am Mikrofilm sind nur Einzelbuchstaben lesbar; der Text ist stark fragm., korrodiert und verwaschen und eine textkritische Auswertung nicht möglich. || Inscriptio (m2), ๆ|ा bei 2,1 und 2,12, Ini.\}

6.-7. Jh. (Schenke) || Herkunft unbekannt || Pergament, 1 Fragm. || 2 Kol. || noch 2 Zeilen (*ca. 26) || 5,19.23.25.29

57 6. Jh. (Schenke) || Herkunft unbekannt || Pergament, 2 Fol. (Doppelblatt) || 2 Kol. || 25 Zeilen $(* 27-28)$ || 13,5-13.15-35 || |ा bei 13,35

58 $\quad$ Lektionar || 12. Jh. ? (Louis) || wohl Weißes Kloster, Sohag (Schüssler) || Papier, 1 Fol. (fragm.) || 1 Kol. || 20 Zeilen (*26) || 19,7-11.12-16.17

59 $\quad$ Sakramentale || 12. Jh. (Louis) || wohl Weißes Kloster, Sohag (Louis), eher Nordägypten (Schüssler) || Papier, 2 Fol. (fragm.) || 1 Kol. || 16-19 Zeilen (*?)|| 16,20-23 || Die Johannes-Perikope ist Lesung für eine im Kindbett verstorbene Frau. 
6./7. Jh. (Schüssler) || Herkunft unbekannt || Pergament, 1 (fragm.) || 1 Kol. (*2) || 8 Zeilen (*30) || 6,1-2.11-12

593 Anno Martyrum (Kolophon; julianisch: 29.08.876-28.08.877) || Deir el-Naqlun (ältere Klosteranlage Raum D.22), Faijum || Pergament, 1 (fragm.) || 1 Kol. (*2) || 34 (recto) und 16 (verso) Zeilen || 21,20-25 || Ini. bei 21,20, Subscriptio, Kolophon

6 Amulett (wegen Faltungsspuren, Schulz), Privatabschrift (Schenke) || 4. Jh. (Schenke) || Herkunft unbekannt || Papyrus, 1 Fol. (fragm.) || 1 Kol. || 20 und 15 Zeilen (*ca. 23) || 20,19-31

8./9. Jh. (Clackson, Schüssler), frühes 9. Jh. (Uljas) || Herkunft unbekannt || Papyrus, 1 Fol. (fragm.) || 2 Kol. || 39-40 Zeilen || 17,8-15.17-26; 18,1.3-14 || Ini. bei *J 18,5 (bei Neqd $2 \epsilon \wedge$ ) und 18,8

Miniatur-Kodex || 9. Jh. ? (Schüssler) || Herkunft unbekannt || Pergament, 1 Fragm. || 1 Kol. || 23 Zeilen || 2,18-25; 3,1-4

5. Jh. (Delattre) || el-Aschmunein || Pergament, 2 Fol. (fragm.) || 1 Kol. || $23-24$ Zeilen || rote Punkte, am Zeilenende bei 11,4 und Zeilenbeginn bei 11,13 || 10,33-42; 11,1-24

9./10. Jh. (Wright/Hubler) || Herkunft unbekannt || Papyrus, 40 Fol. (aus 58 Fragm.) || 2 Kol. || *32 Zeilen || 3,*2.3.9-10; 4,39-41; 5,5-7.32-33; 6,1-2.4-5.7-8. 22.24.43-44; 7,67.12-13.22-24.28.32-33.36-38.47-50; 8,12-14.31.37-38.48-51.53-55; 9,13-22.28-30; 10,6-8.37-38.42; 11,1-3.6-9.13-15.18-21.26-29.32-33.39-41; 13,29-31; 14,8-9; 15,25; $16,4-5.25-26.30-32 ; 17,15-18.22-23 ; 18,21-23.36 ; 19,18-20.32-34 ; 20,4-5.10-11.20-$ 21.25-26.30-31; 21,3-4.*20.21-22.*23.25 || Subscriptio, Kolophon

11. Jh. (Elanskaja) || Herkunft unbekannt || Pergament, 1 Fol. (fragm.) || 1 (*2?) Kol. || 22 Zeilen (*ca. 33) || 18,28-32.35-38 || Ini.

4 Datierung unbekannt || Herkunft unbekannt || Pergament, 1 Fragm. || 2 Kol. || 34 Zeilen || 6,8-13.22-26

Datierung unbekannt || Touton ? (Suciu) || Pergament, 1 Fragm. || 2 Kol. || noch 9 Zeilen (ca. *48-53) || 11,44-46.*51.*52; 12,5-6.15-17 || Ini. mit Abschnitts-Nr. 26 bei 11,54

6 Bilingue (griechisch-sahidisch) || Datierung unbekannt || Herkunft unbekannt || Pergament, 1 Fragm. || 1 Kol. (*2?) || noch 3 Zeilen (*?) || 18,23

Datierung unbekannt || Herkunft unbekannt || Pergament, 1 Fragm. || 2 Kol. || 14 Zeilen (ca. *31-34) || 16,*24.25.29-31; 17,1-3.8-9 || Ini. mit » bei 16,30

Datierung unbekannt || Herkunft unbekannt || Pergament, 1 Fragm. || 1 (*2) Kol. || 14 Zeilen (ca. *29-34) || 2,*25; 3,*1.2.*17.*18.*19 || Ini.

Palimpsest || Datierung unbekannt || Herkunft unbekannt || Pergament, 2 Fragm. || 1 Kol. (*1?) || 5-6 Zeilen (ca. *16-18) || scriptio inferior: unidentifiziert, scriptio superior: 13,29-30.33.36.38; 14,1-2

Datierung unbekannt || Herkunft unbekannt || Pergament, 51 Fol. + 3 Fragm. || 2 Kol. || 8-22 Zeilen (ca. *23) || Johannes folgt im Kodex unüblicherweise den Paulinen. || 1,1-6 
101 6./7. Jh. (Crum, Hyvernat), 8. Jh. (Till, Balestri) || Weißes Kloster, Sohag (Amélineau) || Pergament, 24 Fol. || 2 Kol. || 32 Zeilen || 1,11-12.15; 4,23-25.28-29.33-35.37-40.44-45. 47-49.52-54; 5,3.5-7.18-22.32-34; 6,11.24; 7,6-10.12.17.20-26.28.32-33.35-37; 8,3537.44-46; 10,*11.*12.*13.14-16.*17.*18.19-29; 12,39-43.47-48.50-13,1.3-5 || भाषा, Ini., Zit. $\rightarrow »$

102 6. oder 7. Jh. (Balestri, Cramer), 7. Jh. (Horner, Till), 8. oder 9. Jh. (Crum, Hyvernat) ॥ Weißes Kloster, Sohag (Amélineau) || Pergament, 49 Fol. (teils fragm.) || 2 Kol. || 32- 35 Zeilen || 4,17-36; 9,15-10,7; 16,31-32; 17,2-3.7-8.11 || In der Handschrift sind Tau-

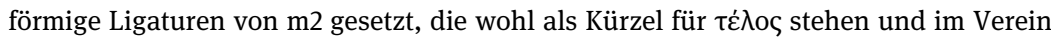
mit पाषा Beginn und Ende von Perikopen bezeichnen. Teil $R^{v}$ wurde mit Makulatur verstärkt (Schrift nicht sicher auszumachen). || बा|ा, Ini.

103 Billingue (griechisch-sahidisch) \| 8. Jh. (Beltz), 8./9. Jh. (Crum, Hyvernat), 10. Jh. (Balestri), 10./11. Jh. ? (Aland für Teil U), 11. Jh. (Till), 11./12. Jh. (Horner) || Weißes Kloster, Sohag (Amélineau) || Pergament, 39 Fol. || 2 Kol. || 31-41 Zeilen || 1,*7.13-14. 18-19.*23.*24.*25.32-34.38-40; 4,42-48.50-5,3.5-11; 6,12-47.50-7,18.41-52; 8,12- 23; 9,7-11,52; 12,38-13,31; 14,29-16,10.29-17,15; 18,23-20,30; 21,15-25 || Teile 0 f. 6 und S mit Makulatur verstärkt (literarische Texte).|| |ा|ा, Ini., Subscriptio, liturgische Glossen und griechisch $20,1-7^{\text {lac }}$

105 Billingue (griechisch-sahidisch) || 6. Jh. (Aland), 7./8. Jh. (Woide), 8./9. Jh. (Horner), 9. Jh. (Till) || Weißes Kloster, Sohag (Amélineau) || Pergament, 45 Fol. (teils fragm.) || 2 Kol. || 35 Zeilen || 3,18-20; 5,13-22.24-25; 6,63-7,1.42-52; 8,12-13.22-9,28; 11,*41.*42. *43.*44.45-48; 12,19-21.36-46; 16,28-29 || Zahlreiche Nachtragungen von ausgelassenem Text am Unterrand. ||

106 8. Jh. (Horner, Petersen), 8. oder 9. Jh. (Balestri), 9. Jh. (Till) || Weißes Kloster, Sohag (Amélineau) || Pergament, 24 Fol. (teils fragm.) || 2 Kol. || 30-32 Zeilen || 1,1-6; 2,133,18; 8,38-9,28; 10,2-16; 12,20-13,8; 16,2-11.13.20-23; 20,26-31; 21,2.4-7 || Фा, Ini. (bei 3,3; 8,46 und 9,27 Фाण in klar abgesetzter Form)

108 10./11. Jh. (Till), 11. Jh. (Horner), ca. 11. Jh. (Balestri) || Weißes Kloster, Sohag (Amélineau) || Pergament, 20 Fol. (fragm.) || 2 Kol. || 27-30 Zeilen || 9,22-40; 10,*40.*41; 11,3. $10-12.15-17.20-22.26-28.31-32.34-37.40-42.44-45.47-48.51-53.55-56.57-12,1$; 13,2-15,20; 16,10-19,38 || Ini., Abschnitts-Nrn.

109 10. Jh. (Horner, Balestri, Till) || Weißes Kloster, Sohag (Amélineau) || Pergament, 27 Fol. || 2 Kol. || 30-33 Zeilen || 8,54-9,18; 15,11-12.16-17; 16,2-6.9-13 || Oxford, Bodlean Library, Copt. c.67 (ehemals Copt. g.3; 11,27-29.32-34, Ini. bei 11,28) gehört gegen Horner nicht zu 109. Da das Blatt bei Drucklegung noch nicht durch das INTF aus der Zusammenstellung von 109 ausgegliedert wurde und ein Abgleich zur eventuellen paläographischen Zuordnung zu einer anderen Handschrift aussteht, wird es in Index und Apparat bei 109 gelistet, doch mit vorangestelltem † markiert. || भाषा, Ini., AbschnittsNr., liturgische Glosse

110 6. Jh. (Beltz), 10. Jh. (Balestri, Horner, Till), 10./11. Jh. (Crum, Hyvernat) || Weißes Kloster, Sohag (Amélineau) || Pergament, 12 Fol. (teils fragm.) || 1 Kol. || 23-27 Zeilen || 1,25-2,18; 3,27-4,3; 8,37-46; 9,12-22; 11,22-28.30-48; 18,38-40; 19,4-6.18-27; 21,2-14 || गाणा ( $\div \div)$, Ini., Abschnitts-Nrn. 
11. Jh. (Schüssler laut Kraus), 11./12. Jh. (Till), 12. Jh. (Ballestri), 13. und 14. Jh. (Horner für verschiedene Teile der Handschrift) || Weißes Kloster, Sohag (Amélineau) || Pergament, 57 Fol. || 2 Kol. || 26-35 Zeilen || 3,22-26.29-30.*31.36; 4,1.6-9.12-36; 6,26-46;

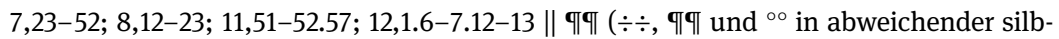
riger Tinte), Ini.

10. Jh. (Balestri, Crum, Hyvernat, Horner, Till) || Weißes Kloster, Sohag (Amélineau) || Pergament, 26 Fol. || 2 Kol. || 34-35 Zeilen || 1,25-4,42; 7,25-45; 8,25-44; 9,7-27; 10,811,10.18-19.24-25.37-57; 16,22-17,8; 20,15-21,2 || Фाण, Ini. (viele, selten aus dem Schriftspiegel herausgesetzt), Abschnitts-Nrn., Glosse ( $\left(\begin{array}{l}\mathrm{p} \\ >\mathrm{d}\end{array}>\right.$ bei 10,22 [Martyrie d, Bedeutung unklar, aber siehe die Perikopenliste in 264 (m2) mit 10,22 für den Sonntag der

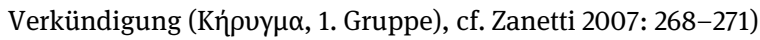

10. Jh. (Till), 11. Jh. (Horner) \| Weißes Kloster, Sohag (Amélineau) || Pergament, 59 Fol. (teils fragm.) || 2 Kol. || 34-40 Zeilen || 1,23-5,3.5-7,52; 8,12-44; 9,6-26; 11,42-12,17.1913,2; 14,10-24.26-29; 18,36-19,31.40-41.*42; 20,2-4.11-21,17 || |ाषा (६, $\div$ und ※.), Ini., Abschnitts-Nrn. (von m2), Zit. $\rightarrow »$ oder $^{\circ}$, Glossen

Billingue (griechisch-sahidisch) || 4. Jh. (Georgi), 5. Jh. (Aland, Gregory), 5. und 6.-8. Jh. (Hyvernat für verschiedene Teile der Handschrift), 5./6. Jh. (Cavalieri, Irigoin, Tisserant, Treu), 6. Jh. (Balestri), 6.-8. Jh. (Clark), 10. Jh. (Amélineau) || Weißes Kloster, Sohag (Amélineau) || Pergament, 32 Fol. (teils fragm.) || 2 Kol. || 27-33 Zeilen || 1,7-11. 15-23; 3,2-10; 4,45-52; 6,21-58.68-7,52; 8,12-23 || बाा Ini.

7. Jh. oder älter, 8. und 11. Jh. (Horner für verschiedene Teile der Handschrift), 7./8. Jh. (Crum, Hyvernat), 8. Jh. (Balestri, Beltz, Leipoldt), 9. Jh. (Till), 10./11. Jh. (Schüssler) \| Weißes Kloster, Sohag (Amélineau) || Pergament, 48 Fol. (teil fragm.) || 2 Kol. || 36-38 Zeilen || 4,27-5,3.5-7,21-22.26-33; 10,*36-*37.*41.42-11,1; 13,18-20.22-26.29-31.3336; 14,29-30; 15,4; 16,*4.5-8.*9.10-11.*12.15.*18.*19.*21; 19,18-35; 20,1.5-*6 || acht Ini. mit $\dot{x}$ und ๆ, sieben Mal bei Jesusworten $(5,19,5,24,5,25,6,26,6,32,6,47,6,53) \|$ Фाषा, Ini.

8. Jh. oder älter und 10. Jh. (Horner für verschiedene Teile der Handschrift), 10. Jh. (Crum, Hyvernat, Balestri, Till) || Weißes Kloster, Sohag (Amélineau) || Pergament, 28 Fol. || 2 Kol. || 37-49 Zeilen || 6,71-7,5.10-16.21-26.30-35; 8,33-42; 9,2-11; 15,3-27; 16,1-17 || $\mid$ |, Ini.

11. Jh. (Horner, Balestri, Till) || Weißes Kloster, Sohag (Amélineau) || Pergament, 26 Fol. || 2 Kol. || 27-31 Zeilen || 4,9-28; 13,20-14,1.*20.21-18,15.18-19.*31.32-19,24 || Innenrand von L/1 durch Makulatur verstärkt (Ps 64,1) || Ф, Ini., Keph., Abschnitts-Nrn.

8./9. Jh. ? (Petersen), 10. Jh. (Till), 10. oder 11. Jh. (Balestri), 11. Jh. (Horner) || Weißes Kloster, Sohag (Amélineau) || Pergament, 17 Fol. || 2 Kol. || 37-40 Zeilen || 7,29-52; 8,129,41 || |ा (meist als », teils als $\div \div$ von m2), Ini., Abschnitts-Nrn.

10./11. Jh. (Crum, Hyvernat), 11. Jh. (Horner, Balestri, Till) \| Weißes Kloster, Sohag (Amélineau) || Pergament, 9 Fol. (teils fragm.) || 2 Kol. || 25-29 Zeilen || 1,24-26.29-32. 33-36.38-40.42-3,4.27-28; 4,9-10; 8,16-34; 9,31-10,10; 12,48-13,17; 14,*17.18-20. 29-31; 17,13-15.18-18,1 || Innenrand von F/1 durch beschriftete Makulatur verstärkt. || भाषा (๕૯, $\div \div)$, Ini. 
127 11. Jh. (Till), 12. Jh. (Balestri, Horner) || Weißes Kloster, Sohag (Amélineau) || Pergament, 28 Fol. || 2 Kol. || 39-44 Zeilen || 8,48-13,1; 17,11-12; 18,3.*4.5-19,40; 20,5-6.*7.

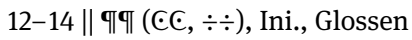

131 6. Jh. (Amélineau), 6.-7. Jh. (Kasser), 7. Jh. (Schüssler), 7.-8. Jh. (Crum), 8. Jh. (Horner), 9.-10. Jh. (Till) || Weißes Kloster, Sohag (Amélineau) || Pergament, 30 Fol. (teils fragm.) || 2 Kol. || 35 Zeilen || 11,46-12,12.16-*18.21-24.27-29; 16,32-17,1.*2.3-4.*5-*6. 7-*19; 21,*13.14-25 || Фा|ा, Ini. (teils *.), Keph., can., Subscriptio, Stichoi-Angabe

132 13. Jh. (Horner, Balestri) || Weißes Kloster, Sohag (Amélineau) || Pergament, 24 Fol. || 1 Kol. || 19-26 Zeilen || 7,18-28; 12,48-13,8 || Ini., Abschnitts-Nr.

134 10./11. Jh. (Till), 11. Jh. (Horner) || Weißes Kloster, Sohag (Amélineau) || Pergament, 22 Fol. || 2 Kol. || 37-40 Zeilen || 1,1-27; 7,29-33.39-43.48-52; 8,12-14; 9,19-10,10; 11,4012,13 || Inscriptio, Ini.

136 8. Jh. (Kasser), 9. Jh. (Till) || Weißes Kloster, Sohag (Amélineau) || Pergament, 18 Fol. (teils fragm.) || 2 Kol. || 32 Zeilen || 1,43-51; 2,1-4,9; 5,14-30; 6,43.*44.58.*61.*62.63-65. *66.67.68-7,10.28-52; 8,12-10,12; 11,50-12,4.6-13.16-19.21-28.30-34.36.40-41.44. *45.46; 20,31; 21,16 || |ाषा, Ini.

137 11. Jh. (Horner, Balestri) || Weißes Kloster, Sohag (Amélineau) || Pergament, 18 Fol. || 3,4-6.7-17 || Ini.

139 9. Jh. (Till), 9.-10. Jh. (Kasser), 11. Jh. ? (Horner) || Weißes Kloster, Sohag (Amélineau) || Pergament, 17 Fol. (teils fragm.) || 2 Kol. || 26-32 Zeilen || 1,2-11.13-16.18-25.28-30.33$34.38-40 ; 3,23-4,9 ; 5$,*42.43-45.47; 6,1-13.37-38.51-52.65-7,10.44-52; 8,12-20.*36. $37-49 ; 11,44-12,2.21-29.31-38 ; 13,5-34.36-15,5.22-16,16 ; 18,29-19,3.35-36.39-42$; 20,1-7.12 || Ini., Abschnitts-Nrn.

140 6.-7. Jh. (Crum, Hyvernat), 8. und 10. Jh. (Horner für verschiedene Teile der Handschrift), 8./9. Jh. (von Lemm), 10. Jh. (Till) || Weißes Kloster, Sohag (Amélineau) || Pergament, 23 Fol. || 2 Kol. || 36-40 Zeilen || 17,3-19,23 || Ini. (außer bei 19,7 stets mit ※.)

141 9. Jh. (Elanskaja, von Lemm), 10. Jh. (Till, Hyvernat), 10./11. Jh. (Schüssler), 11. Jh. (Horner), 11.-12. Jh. (Kasser) || Weißes Kloster, Sohag (Amélineau) || Pergament, 15 Fol. (teils fragm.) || 1 Kol. || 19-22 Zeilen || 3,17-21.22-27.28-4,5.27-39; 6,29-41; 7,4-18; $8,37-40.42-44.58-9,1.6-7.40-10,11 ; 12,25-35.48-13,9 ; 14,17-18 . * 19-20.22-24 ; 16,1-$ 24; $18,12-22.40-19,11$ || Фा|ा, Ini.

142 Palimpsest || scriptio superior (J): 10. Jh. (Kasser), 11. Jh. (Till), 12./13. Jh. (Horner) \| Weißes Kloster, Sohag (Amélineau) || Pergament, 15 Fol. || 1 Kol. || 19-24 Zeilen || scriptio inferior: unidentifiziert, scriptio superior: 4,6-11.12-*18; 9,28-*31.34-38; 10,16.21$11,6.34-46.48 .49 . * 50 ; 12,8-18.36-37.41-42 ; 13,7-38 ; 14,1-2.3-20 ; 17,11-13 . * 14.15-20$; 18,36-37.40; 19,1-4.*24-26.*27.*29.30-31 || Ini., Abschnitts-Nrn.

143 7./8. Jh. (Crum/Hyvernat), 9. und 10. Jh. ? (Horner für verschiedene Teile der Handschrift); 10./11. Jh. (Balestri) || Weißes Kloster, Sohag (Amélineau) || Pergament, 14 Fol. (teils fragm.) || 2 Kol. || 39-43 Zeilen || 20,8-11.17.24-25.29-31 || बा bei 20,11 
Datierung unbekannt || Herkunft unbekannt || Pergament, 10 Fol. (fragm.) || 2 Kol. || 517 Zeilen (ca. *36-40) || 3,*10.*11.*15.*16.*17.20-22.26-27; 15,*7.8-*9.15 || Ini. bei 3,26 (єіс гннте)

10. Jh. (Horner) || Weißes Kloster, Sohag (Amélineau) || Pergament, 7 Fol. || 2 Kol. || 29 Zeilen || 4,36-38.*39.40-41.44-45.47-49.*50; 11,56-57; 12,1.2-3.5-7.9-11.19-34.4042.43-46.47-49.50; 13,1-2.*17.18-31; 16,*15.16-33; 17,1.3-6 || |ा, Ini., liturgische Glossen (von $\mathrm{m} 2$ )

10. Jh. (Till), 11. Jh. (Balestri, Horner) || Weißes Kloster, Sohag (Amélineau) || Pergament, 6 Fol. (teils fragm.) || 2 Kol. || 27-32 Zeilen || 6,35-37.39-42.44-46.48-51; 8,2335.37-39; 11,32-33.36-39; 18,31-19,4.17-20,2 || Ini., Abschnitts-Nrn.

7. Jh. ? (Horner) || Weißes Kloster, Sohag (Amélineau) || Pergament, 5 Fol. (aus 6 Teilen, fragm.) || 1 Kol. || 20-22 Zeilen || 7,33-35.37-38.*40.41-8,34 || Ini.

10. Jh. (Horner) || Weißes Kloster, Sohag (Amélineau) || Pergament, 5 Fol. (fragm.) || 2 Kol. || 32-36 Zeilen || 6,61-7,1.3-8.*9.10-23.*24.*25.26-28.*29-*30.31-32.*33-*34.3540; 15,6-10.13-16.18-20.22-26 || बाषा, Abschnitts-Nrn.

6. Jh. (Crum/Bell) || Wadi Sarga (Crum/Bell) || Pergament, 4 Fol. (2 Doppelblätter) || 2 Kol. || 16-18 Zeilen (*18/19) || 21,7-9.10-25 || Subscriptio

13. Jh. (Horner) || Weißes Kloster, Sohag (Amélineau) || Pergament, 4 Fol. (teils fragm.) || 2 Kol. || 35-42 Zeilen || 1,47-4,22; 5,24-6,5 || Ini., Abschnitts-Nrn.

4. Jh. (Horner) || Herkunft unbekannt || Papyrus, 4 Fol. (fragm.) || 1 Kol. || 14-16 Zeilen (*19/20) || 20,2-8.*9.10-16.17-22.23-28.*29

ca. 10. Jh. (Balestri), 10. Jh. (Horner) || Weißes Kloster, Sohag (Amélineau) || Pergament, 3 Fol. || 1 Kol. || 27-28 Zeilen || 9,11-27; 10,25-11,2; 12,23-39 || Ini.

11. Jh. (Balestri, Horner) || Weißes Kloster, Sohag (Amélineau) || Pergament, 3 Fol. || 2 Kol. || 28-30 Zeilen || 2,2-20.23-3,6; 9,3-10,1 || Ini., Abschnitts-Nrn.

5. Jh. (Kahle), 7. Jh. (Horner) || Deir el-Bala'izah || Pergament, 2 Fol. || 2 Kol. || 29 Zeilen || $2,24-3,15$

6. Jh. (Beltz), 6.-7. Jh. (Leipoldt) || Herkunft unbekannt || Pergament, 2 Fol. (aus 3 Teilen) || 2 Kol. || 5-23 Zeilen (ca. *24) || 3,2-4.5.*6.*7.8-10.12-16; 9,22-25.28.31-34

5. Jh. (Horner), ca. 6. Jh. (Leipoldt) || Herkunft unbekannt || Pergament, 2 Fol. || 1 Kol. || 22 Zeilen || 13,2-3.8-9

180 5. Jh. (Beltz) || Herkunft unbekannt || Pergament, 2 Fol. (= Doppelblatt aus 5 Fragm.) || 1 Kol. || ca. *14 Zeilen || 13,3-8.29-30.32.33.*34

10. ? und 11. Jh. (Horner für verschiedene Teile der Handschrift) || Herkunft unbekannt || Pergament, 7 Fol. (fragm.) + 7 Fragm. || 2 Kol. || 40-42 || 1,4-12.13-17.19-25.29-33.3742. 44-49.*50.51-2,8.10-15.19-24; 3,2-6.8-15.18-23.27-31.34-4,5.9-13.18-22. 25-30. 34-38 || Фा|, Ini. (vermerkt nach von Lemm 1885) 
|| 2 Kol. || 21-28 Zeilen || scriptio inferior: Martyrium und Wunder des Hl. Philotheus von Antiochien (faijumisch), scriptio superior: 12,36-13,2; 19,38-21,23 || Ini., Abschnitts-Nrn. (von m2)

184 Datierung unbekannt || Herkunft unbekannt || Papyrus, 4 Fol. (aus 10 Fragm.) || 1 Kol. || 2-8 Zeilen $(* 18-19)|| 15, * 16 . * 17.18-19.20-* 21.24 .25 .27-16,2 . * 3.4 .5-6.7-8 . * 13 . * 16$

185 Palimpsest || Datierung unbekannt || Herkunft unbekannt || Pergament, 4 Fol. (fragm.) || 2 Kol. || 5-14 Zeilen (*ca. 20-24) || Als Material wurden Fragm. aus zwei unterschiedlichen Handschriften wiederverwendet. || 8,31-33.35-36; 13,38-14,*1.*2.*9.*10

187 8. Jh. (Horner), 8./9. Jh. (Crum/Hyvernat) || Herkunft unbekannt || Pergament, 3 Fol. || 2 Kol. || 25-26 Zeilen || 3,29-4,9.35-47; 11,*33.34-47 || Фा (๔) bei 4,1

190 12. Jh. (Horner) || Weißes Kloster, Sohag (Schüssler) || Pergament, 3 Fol. (fragm.) || 2 Kol. || *35-38 Zeilen || 2,14-15; 3,14-16.17-18; 4,7-9.14-21.23-31.32-38.40-46 || Ini.

193 Miniatur-Kodex || 6. Jh. (Beltz) || el-Aschmunein (Beltz) || Pergament, 2 Fol. (Doppelblatt) || 1 Kol. || 13-14 Zeilen || 4,1-10

194 6.-7. Jh. (Pezin) || Herkunft unbekannt, angekauft in Luxor (cf. BC) || Pergament, 2 Fol. (fragm.) || 1 Kol. || 7-9 Zeilen (ca. *22) || 5,34-35.39-40.45-47; 6,5-7

195 7. Jh. (Till, Kasser) || Herkunft unbekannt || Pergament, 2 Fol. (fragm.) || 2 Kol. || 6-11 Zeilen $(* 27 / 28)$ || 3,32-33; 4,9

196 7. Jh. oder älter (Horner) || Herkunft unbekannt || Pergament, 2 Fol. || 2 Kol. || 31 Zeilen || $2, * 3.4-3,14$

197 10. Jh. (Balestri, Horner) || Weißes Kloster, Sohag (Orlandi) || Pergament, 2 Fol. (fragm.) || 2 Kol. || 36-39 Zeilen || 7,35-52; 8,12-25; 10,21-11,13 || Ini., Keph., Glosse, Rubrizierung (10,22 und Beginn von 11,1 in Rot)

199 11. Jh. (Horner) || Weißes Kloster, Sohag (Amélineau) || Pergament, 2 Fol. (fragm.) || 2

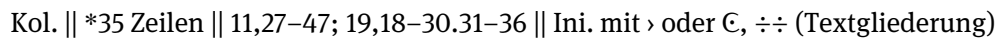

205 4. Jh. (Roca-Puig) || Herkunft unbekannt || Pergament, 1 Fol. (fragm.) || 1 Kol. (*2) || 6 Zeilen (ca. *48-50) || 18,26-28; 19,10-11

207 5.-6. Jh. (Kahle), 6. Jh. (Horner, Schüssler) || Deir el-Bala'izah || Pergament, 1 Fol. (fragm.) || 2 Kol. || max. 26 Zeilen || 15,14-22.24-26 || || bei 15,17 (єтетмемере)

209 1. Hälfte 5. Jh. (Roca-Puig) || Herkunft unbekannt || Pergament, 1 Fol. (fragm.) || 1 Kol. (*2) || 13 Zeilen (*ca. 21-23) || 17,19-21.22-23

210 5. Jh. (Horner) || Weißes Kloster, Sohag (Schüssler) || Pergament, 1 Fol. (fragm.) || 2 Kol. || 11-13 Zeilen $(* 21-22)$ || 7,50-52; 8,12.*13.*14.*15.16.*17.*18 || Laut BC 4.2: 126 sei bei 8,12 ein getilgtes $\mathrm{P}$, doch liegt ein spezieller Paragraphos vor. \| |ाषा bei *7,52 (unsicher), 8,12 und $8,17(>)$

215 6. Jh. (Beltz) || Herkunft unbekannt || Pergament, 1 Fol. (aus 2 Teilen, fragm.) || 1 Kol. (*2) || 18-19 Zeilen (ca. *26/27) || 1,41-42.*43.50-51 || || und Ini. bei 1,43

216 6. Jh. (Beltz), 6.-7. Jh. (Leipoldt, Schüssler), 7. Jh. oder älter (Horner) || Herkunft unbekannt || Pergament, 1 Fol. || 1 Kol. || 19 Zeilen || 8,21-27 
217 6. Jh. (Till) || Herkunft unbekannt || Pergament, 1 Fol. (fragm.) || 1 Kol. (*2) || 10-11 Zeilen (*ca. 33-35) || 19,40-42; 20,4-5.*6

218 6. Jh. (Horner) || Weißes Kloster, Sohag (Schüssler) || Pergament, 1 Fol. (fragm.) || 1 Kol. || 25 Zeilen || 9,22-38 || || und Ini. bei 9,26

219 Palimpsest || scriptio inferior (J): 5.-7. Jh. (Crum/Bell), scriptio superior (Act): 6. Jh. ? (Schüssler), 6. Jh. (Crum) || Wadi Sarga (Crum/Bell) || Pergament, 1 Fol. || 2 Kol. || scriptio inferior: 25 (са. *35-36) || scriptio inferior: 1,*17.*18.*19.*20.*21.*22.*23.*24.*25. *26.*27.*28.*29.30-32.*33, scriptio superior: Act

231

$299^{\mathrm{L}}$ zugeordnet, cf. dort

11. Jh. ? (Horner), 11. Jh. (Schüssler) || Weißes Kloster, Sohag (Amélineau) || Pergament, 1 Fol. (fragm.) || 2 Kol. || 30-31 Zeilen || 7,18-40 || Ф|णा, Ini.

11. Jh. ? (Horner) || Weißes Kloster, Sohag (Amélineau) || Pergament, 1 Fol. || 2 Kol. || 32-33 Zeilen || 18,3-26 || Ini.

11. Jh. (Balestri, Horner) || Weißes Kloster, Sohag (Schüssler) || Pergament, 1 Fol. (fragm.) || 1 Kol. (*2) || 25-26 Zeilen (ca. *30) || 21,18-22.23-25 || Ini. bei 21,20, Subscriptio

11. Jh. (Till), 11.-12. Jh. (Kasser, Schüssler) || Herkunft unbekannt || Pergament, 1 Fol. || 2 Kol. || 30-31 Zeilen || 8,33-54 || Ini.

6. Jh. (Schüssler) || Herkunft unbekannt || Papyrus, 1 Fragm. || 1 Kol. || 7-8 Zeilen (ca. *15) || 13,2-3.5-7 || *बा $(13,3)$

6. Jh. (Schüssler) || Herkunft unbekannt || Papyrus, 1 Fol. (aus 4 Teilen) || 1 Kol. || 17 Zeilen || 6,21-27.*28

7. Jh. (Schüssler) || Herkunft unbekannt || Papyrus, 1 Fol. (fragm.) || 2 Kol. || max. 8 Zeilen (ca. *35) || 5,3-6.13-*14.*19.20.25-26

7. Jh. (Schüssler) || Herkunft unbekannt || Papyrus, 1 Fragm. || 2 Kol. || *32 Zeilen || 7,*29.30-49 || |ा|, Ini., Abschnitts-Nr.

10. Jh. (Schüssler) || Herkunft unbekannt || Pergament, 1 Fragm. || 1 Kol. (*2) || 10 Zeilen (ca. *23) || 2,*6.7-8.*9.10-11

9./10. Jh. (Schüssler) || Weißes Kloster, Sohag (Amélineau) || Pergament, 1 Fragm. || 1 Kol. $\left(*{ }^{\text {vid. }) ~|| ~} 6\right.$ Zeilen $($ ca. $* 25) \|$ || 21, *14.15.18 || Ini. bei 21,15

10. Jh. (Schüssler) || Weißes Kloster, Sohag (Amélineau) || Pergament, 1 Fragm. || 1 Kol. (*2) || 6-8 Zeilen (*32-35) || 20,25-26; 21,*9.10.*11 || Ini. bei 20,26

10. -11. Jh. (Schüssler) || wohl Faijum (Layton) || Pergament, 1 Fragm. || 1 Kol. (*2 $2^{\text {vid. }) ~|| ~} 15$ Zeilen (*34) || 10,24-28.34-38

11. Jh. (Schüssler) || Weißes Kloster, Sohag (Schüssler) || Pergament, 1 Fragm. || 2 Kol. || *max. 47 Zeilen || 18,8-23.24-38 || Ini.

Datierung unbekannt || Herkunft unbekannt || Pergament, 1 Fragm. || 2 Kol. || 24-30 Zeilen (ca. *30) || 13,13-17.18-21.22-26.27-32 || ๔ (nur Reste, wohl zu Ini. bei 13,14) 
268 5. Jh. (Heidelberg, UB) || Herkunft unbekannt || Pergament, 1 Fragm. || 2 Kol. || 21-22 Zeilen (*22) || 20,16-18.19-21.21-24.25-26 || Ini. bei 20,24 (nur leicht ausgesetzt)

270 9./10. Jh. (Schüssler) || Herkunft unbekannt || Pergament, 1 Fragm. || 1 Kol. || 16 Zeilen || 7,27-32 || Ini. bei 7,28 (Kleeblattkreuz), 7,30 und 7,32 ( $\div$ )

271 6. Jh. (Kasser) || Wadi Sarga (Crum/Bell) || Pergament, 1 Fragm. || 2 Kol. || 5-12 Zeilen (ca. *35) || 20,25-26.31-21,2.6-7.12-13 || Ini. mit शाषा bei 21,1 und *21,13

275 9. Jh. ? (Schüssler) || Herkunft unbekannt || Pergament, 1 Fragm. || 2 Kol. || 5-17 Zeilen (ca. *38-40) || 15,27-16,3.7-8.15-16.19-21

276 9. Jh. ? (Schüssler) || Herkunft unbekannt || Pergament, 1 Fragm. || 1 Kol. (*2) || 20 Zeilen (ca. *36) || 6,23-26.27-30

285 14. Jh. (Horner) || Herkunft unbekannt || Papier, 23 Fol. || 1 Kol. || 17-19 Zeilen || 7,13-19; 8,20-33.40-11,32; 12,9-25 || Ini., Keph., Abschnitts-Nrn., can.

286 13.-14. Jh. (Burmester) || Deir Abu Maqar, Wadi Natrun (Schüssler) || Pergament, 10 Fol. || 1 Kol. || 14-18 Zeilen || 13,10-15; 14,21-26.30-16,13 || Ini., Abschnitts-Nrn.

291 Lektionar (Sabbato-Kyriakai) || 12./13. Jh. (Joussen), 13. Jh. (Horner) || Herkunft unbekannt || Papier, 38 Fol. || 2 Kol. (und 1 = Ps) || 37 Zeilen || 4,5-30; 5,1-14; 7,14-17

292 $\quad$ Pascha-Lektionar || 13. Jh. (Horner) || Herkunft unbekannt || Papier, 37 Fol. (1 halb) || 2 Kol. || 29-31 Zeilen || 17,6-26; 18,1.2.6-10.15-40; 19,1-12 || Abschnitts-Nrn. (ungenau)

293 Lektionar (Sabbato-Kyriakai und Hochfeste) || 8.-9. Jh. (Willis), ca. 10. Jh. (Schüssler), 10.-11.Jh. (Till), 11.-12. Jh. (Horner) || Weißes Kloster, Sohag (Amélineau) || Pergament, 32 Fol. (teils fragm.) || 2 Kol. (und 1 = Ps) || 26-33 Zeilen || 1,1-13; 4,19-29; 6,62-69; 21,1-11.15-20 || liturgische Rubrik

294 Lektionar (Sabbato-Kyriakai) || 11. Jh. (Crum/Hyvernat), 14. Jh. (Horner) \| Weißes Kloster, Sohag (Amélineau) || Papier, 17 Fol. (aus 20 Teilen) || 2 Kol. || 28-30 Zeilen || 2,6-11.18-22.23-25; 6,8-9.15-26; 10.31-32; 15,*3-4

295 Lektionar (Marien- und Hochfeste) || 8.-9. Jh. (Willis), 9.-10. Jh. (Schüssler), 10. Jh. (Balestri, Till), 11.-13. Jh. (Horner) || Weißes Kloster, Sohag (Amélineau) || Pergament, 20 Fol. (teils fragm.) || 2 Kol. (und 1 = Ps) || 28-30 Zeilen || 1,1-14; 2,12-22 || liturgische Rubriken

296 Lektionar || 9. Jh. (Schüssler), 10. Jh. (Beltz), 10.-11. Jh. (Leipold), 11. Jh. (Till), 11.-13. Jh. (Horner) || Weißes Kloster, Sohag (Amélineau) || Pergament, 14 Fol. (teils fragm.) || 2 Kol. (und 1 = Ps) || 21-26 Zeilen || 3,26-28; 12,6-8

297 $\quad$ Evangelistar || 9.-10. Jh. (Crum/Hyvernat), 10. Jh. (Horner, Till) \|| Weißes Kloster, Sohag (Amélineau) || Pergament, 9 Fol. (aus 11 Teilen) || 3 Kol. || 4-28 Zeilen (*36-38) || 1,45-46; 4,19-20; 14,26-27.28-15,1.3-6.7-11.26-16,2.4-9.13-15.19; 17,8-9

298 $\quad$ Pascha-Lektionar \| 10. und 11. Jh. (Till für verschiedene Teile der Handschrift), 11. Jh. (Balestri, Horner), 11.-12. Jh. (Horner) || Weißes Kloster, Sohag (Amélineau) || Pergament, 11 Fol. || 2 Kol. (und 1 = Ps) || 24-28 Zeilen || 19,1-16 
299 $\quad$ Pascha-Lektionar || 10. Jh. (Schüssler), 10. und 11. Jh. (Till für verschiedene Teile der Handschrift), 11.-12. Jh. (Kasser), 14. Jh. (Horner) || Weißes Kloster, Sohag (Amélineau) || Pergament, 9 Fol. (teils fragm.) || 2 Kol. (und 1 = Ps) || *35-36 Zeilen || Die ehemals eigenständige 231 wurde $299^{\mathrm{L}}$ zugeordnet. || 15,10-16,8 || liturgische Rubrik

$300^{\mathrm{L}} \quad$ Lektionar || 9. Jh. (Schüssler), 11. Jh. (Till), 13.-14. Jh. (Horner) || Weißes Kloster, Sohag (Amélineau) || Pergament, 8 Fol. (teils fragm.) || 1 Kol. || 19-22 Zeilen (*20-22) || 12,2627

301 $\quad$ Lektionar (Sabbato-Kyriakai und Festtage) || 10. Jh. (Horner), 10. oder 11. Jh. (Balestri) \| Weißes Kloster, Sohag (Amélineau) || Pergament, 6 Fol. (aus 8 Teilen) || 2 Kol. || 6-37 Zeilen (*37-40) || 4,5.6-11.13-15; 6,15-17.18-24; 12,12-17.18-23

302 Lektionar || 9. Jh. (Willis), 10.-11. Jh. (Balestri, Till, Schüssler), 11.-12. Jh. (Horner) \| Weißes Kloster, Sohag (Amélineau) || Pergament, 9 Fol. (aus 12 Teilen) || 2 (und 1 = Ps) || 15-30 Zeilen (*26-30) || 7,37-45; 17,4-10; 20,22-23

305 Lektionar || 834 Anno Martyrum (Kolophon; julianisch: 29.08.1117-28.08.1118 A. D.), 10. Jh. (Till, Schüssler), 12. Jh. (Horner) || Weißes Kloster, Sohag (Amélineau) || Pergament, 7 Fol. || 2 Kol. (und 1 = Ps) || 31 Zeilen || 7,37-46; 12,35-42

306 ${ }^{\mathrm{L}}$ Lektionar || 9.-10. Jh. (Schüssler), 14. Jh. (Horner) || Weißes Kloster, Sohag (Amélineau) || Pergament, 4 Fol. || 1 Kol. || 22-24 Zeilen || 3,1-8

308 $\quad$ Lektionar || 5. Jh. (Beltz, Schüssler), || Herkunft unbekannt || Pergament, 3 Fol. (fragm.) || 2 Kol. (und 1 = Ps) || 6-25 Zeilen (*23-25) || 20,2-3.15-16

310 Lektionar || 11.-12. Jh. (Balestri, Schüssler), 12.-13. Jh. (Horner) || Weißes Kloster, Sohag (Amélineau) || Pergament, 3 Fol. || 2 Kol. (und 1 = Ps) || 27-29 Zeilen || 4,6-14

313 Lektionar || 6.-7. Jh. (Maspero), 7. Jh. (Crum, Layton, Schüssler, Willis) || Herkunft unbekannt || Papyrus, 2 Fol. (aus 3 Teilen) || 1 Kol. || 22 Zeilen (*?) || 20,1-2

$317^{\mathrm{L}} \quad$ Lektionar || 10. Jh. (Horner) || Weißes Kloster, Sohag (Amélineau) || Pergament, 1 Fol. (fragm.) || 2 Kol. || 7-29 Zeilen (*ca. 32) || 21,7-11.11-12

318 $\quad$ Lektionar || 10. Jh. (Till) || Herkunft unbekannt || Pergament, 1 Fol. (fragm.) || 1 Kol. (*2?) || 5 Zeilen $(* ?)|| 6,47-48$

324 $\quad$ Lektionar || 13. Jh. (Horner) || Weißes Kloster, Sohag (Amélineau) || Pergament, 1 Fol. || 2 Kol. || 14-23 Zeilen || 11,5-9 (Textabbruch im Vers vor dem Kolumnenende)

326 ${ }^{\mathrm{L}} \quad$ Lektionar || Datierung unbekannt || Weißes Kloster, Sohag (Amélineau) || Pergament, Fol. 1 (fragm.) || 1 Kol. (*2) || 32-33 Zeilen (*33-34) || 2,*5.6-11

330 $\quad$ Lektionar || Datierung unbekannt || Herkunft unbekannt || Papyrus, 1 Fragm. || 1 Kol. $\left(* 2^{\text {vid. }}\right)|| 3$ Zeilen $(* ?) \| 18,37$

335 $\quad$ bilingues Evangelistar (griechisch-sahidisch) || 8. und 9. Jh. (Aland für verschiedene Teile der Handschrift), 8. und 9.-10. Jh. (van Haelst für verschiedene Teile der Handschrift), 8. und 10. Jh. (Horner für verschiedene Teile der Handschrift), 10. Jh. (Till) ॥ Weißes Kloster, Sohag (Amélineau) || Pergament, 8 Fol. (teils fragm.) || 3 Kol. || 9-22 Zeilen (*33) || 19,20-21.23-24; 20,1.3-5.7-9.12-13.15-18.24-31 || liturgische Rubrik 
336 ${ }^{\mathrm{L}} \quad$ Lektionar (Psalmtexte als griechisch-sahidische Bilingue) $\|$ 6. Jh. (van Haelst), 10. Jh. (Horner), 10./11. Jh. (Till) || Weißes Kloster, Sohag (Amélineau) || Pergament, 7 Fol. (teils fragm.) || 2 Kol. 4-32 (*32) || 14,26-27.28-15,9; 20,28-31 || liturgische Rubriken

$337^{\mathrm{L}} \quad$ bilingues Lektionar (griechisch-sahidisch) \| 7., 8. und 11. Jh. (Aland für verschiedene Teile der Handschrift), 9.-10. Jh. (van Haelst), 10. Jh. (Horner), 10.-11. Jh. (Balestri) ॥ Weißes Kloster, Sohag (Amélineau) || Pergament, 6 Fol. (teils fragm.) || 2 Kol. (und 1 = Ps) || 16-32 Zeilen (*31-32) || 19,16b-24 || liturgische Rubrik

339 $\quad$ bilingues Evangelistar (griechisch-sahidisch) || 10. Jh. (Horner), 10./11. Jh. (van Haelst), 12. Jh. (Aland) || Weißes Kloster, Sohag (Amélineau) || Pergament, 7 Fol. (fragm.) || 2 Kol. || 18-34 Zeilen (*30-34) || 4,5-12; 10,12-15.16-18; 20,8-18 || liturgische Rubriken

$340^{\mathrm{L}} \quad$ bilingues Lektionar (griechisch-sahidisch) \| 7., 9. und 10. Jh. (Aland für verschiedene Teile der Handschrift), 7./8. Jh. (de Ricci/Wilson), 8. und 11. Jh. (van Haelst), 9. Jh. (Horner), 10.-11. Jh. (Clark) || Weißes Kloster, Sohag (Amélineau) || Pergament, 8 Fol. (fragm., darunter 1 Doppelblatt) || 2 Kol. || 2-33 (*ca. 32-33) || 20,1.25-26.29-30 || liturgische Rubrik (zu griechisch 1,1)

342 $\quad$ bilingue liturgische Handschrift (griechisch-sahidisch) || 7., 8., 9. und 13. Jh. (Aland für verschiedene Teile der Handschrift), 8. und 13. Jh. (van Haelst für verschiedene Teile der Handschrift), 10. Jh. (Balestri/Hyvernat), 11. Jh. (Beltz) || Weißes Kloster, Sohag (Amélineau) || Pergament, ca. 11 Fol. || 2 Kol. || 10-30 Zeilen (*meist 30) || Die Zusammenstellung der Handschrift ist revisionsbedürftig. Der neueste Bearbeitungsversuch ist fragwürdig (Atanassova 2011). || 20,24-27.28-31

$343^{\mathrm{L}} \quad$ bilingues Evangelistar (griechisch-sahidisch) || vor 988 A. D. (Layton) || Herkunft unbekannt || Pergament, 2-3 Fol. (aus 5 Teilen) || 2 Kol. || *28-31 Zeilen || 7,38-40.42-44

347 ${ }^{\mathrm{L}} \quad$ bilingues Evangelistar (griechisch-sahidisch) || 9. Jh. (Aland), 9./10. Jh. (Crum/Hyvernat, van Haelst), 10. Jh. (Turner) || Weißes Kloster, Sohag (Amélineau) || Pergament, 1 (aus 5 Fragm.) || 3 Kol. || 5-17 Zeilen (*38) || 6,1-3.*4.5-7.8-9.10-12.13-14 || liturgische Rubrik

349 $\quad$ bilingues Lektionar (sahidisch-arabisch) || 13. oder 14. Jh. (Balestri), 14. Jh. (Horner) \| Weißes Kloster, Sohag (Orlandi) || Papier, 4 Fol. || 2 Kol. || $24-25$ Zeilen || 13,21-30; 17,17-26 || liturgische Rubrik, arabischer Kolophon

350 bilingues Lektionar (griechisch-sahidisch) || 8. Jh. (Aland, Till, van Haelst) || Herkunft unbekannt || Pergament, 1 Fragm. || 1 Kol. (*2) || 9-10 Zeilen (*25-27) || Bestimmung nicht gänzlich sicher. $\| 7,7$

352 bilingues Lektionar (griechisch-sahidisch) || Datierung unbekannt || Weißes Kloster, Sohag (Amélineau) || Pergament, 1 Fragm. || 1 Kol. (*2) || 12-13 Zeilen (*ca. 32) || Bestimmung nicht gänzlich sicher. || 19,16b-17.20.*21

369 11. Jh. (Schüssler) || Weißes Kloster, Sohag (Schüssler) || Pergament, 1 Fragm. || 2 Kol. || ca. *47 Zeilen || 20,4.12-25.*26.*31 || Ini., Abschnitts-Nr.

370 4. Jh. (Ann Arbor, UML) || Herkunft unbekannt || Papyrus, 52 kleine und große Fragm. (teils aus kleineren Fragm. zusammengesetzt) || 1 Kol. || *ca. 22-26 Zeilen || 14,11.*12.13. *14.16.17.18-20.21.22-24.26-28.30-31; 15,1-2.4-6.*7.8.9-12.13.15-16.*18.19-22.*23. 
24-27; 16,1-13.14.15-16.17-20.21.*24.25.*30.31-32.33; 17,1.2-5.6-7.8-11.*12.13-15.18. *19.*20; 18,8-10.*11.15.19-20.23-24; 21,1-8 || Фा bei 14,31 (bei тоүмтнүтN)

393 $3^{\text {var. }}$ Amulett || 7.-9. Jh. (Emmenegger) || Herkunft unbekannt || Pergament, 1 Fol. || 1 Kol. || 13 Zeilen || 1,1; 21,25 || Text und Orthographie entsprechen 9, nur orw am Beginn von 21,25 fehlt und statt гемкегвнүе steht гмкегвнүе. Wie im Mehrheitstext ist $\Delta €$ vorhanden.

396 ${ }^{\mathrm{L}} \quad$ Typikon, Palimpsest || Datierung unbekannt || Herkunft unbekannt || Pergament, 1 Fol. || 22-23 Zeilen || scriptio inferior: unidentifiziert, scriptio superior: Typikon für Samstage und Sonntage des Hathor mit 1,6.43; 5,33; 12,29 (nur Incipits)

402 „Bibelorakel“ || 5.-7. Jh. (Jones) || Herkunft unbekannt || Pergament, 1 Fol. (fragm.) || 2

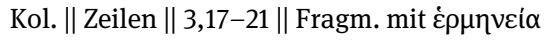

407 $\quad$ Lektionar || Datierung unbekannt || Epiphanius-Kloster, Theben (Crum) || Pergament, 1 Fol. (fragm.) || 2 Kol. (und 1 = Ps) || *32-33 Zeilen || 10,12-18

408 $\quad$ Palimpsest || Datierung unbekannt || Herkunft unbekannt || Pergament, 1 Fol., (fragm.) || scriptio inferior: 2 Kol., scriptio superior: 1 Kol. || scriptio inferior: 15-23 Zeilen (*?), scriptio superior: 7 Zeilen || scriptio inferior: Lektionar mit 19,25-27, scriptio superior: Subscriptio und Kolophon

411 L liturgische Privatabschrift (Schulz) || 10.-11. Jh. (Schulz), 16. Jh. (Beltz) || Herkunft unbekannt || Pergament, 1 Fragment || 1 Kol. || noch 9 Zeilen (*?) || 1,1-5 || liturgische

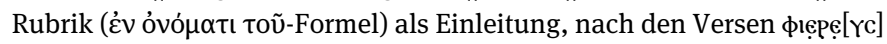

440 polyglotte liturgische Handschrift/Processionale zum Wüstenfest des Apa Schenute (griechisch-sahidisch-bohairisch-arabisch) || 13. Jh. (Boud'hors), 15./16. Jh. (Timbie), 16. Jh. (Quatremère) || Weißes Kloster, Sohag (Boud'hors: „rapportés de Chypre“) || Papier, noch 78 Fol. || 1 und 2 Kol. (teils Sprachen parallel) || *5-36 Zeilen || 12,12-36 (Perikope endet mit м⿻)не пте поүоєім)

452 $2^{\text {var. }}$ Amulett || 7./8. Jh. (Ann Arbor, UML) || Herkunft unbekannt || Pergament, 1 Fol. || 1 Kol. || 16 Zeilen || 1,1 || Inscriptio

453 Zitate (in einer Homilie?) || 7.-8. Jh. (Heidelberg, UB) || Herkunft unbekannt || Papyrus, 5 Fragm. || 2 Kol. || noch 3-12 Zeilen (*?) || 18,8 und 18,36 || Ini. und $\div$ bei 18,36 (ene оүево入)

454 Datierung unbekannt || Herkunft unbekannt || Pergament, 1 Fragment || 1 Kol. (*2) || 3 Zeilen (*?) || 3,36; 4,*1.25

455 Datierung unbekannt || Weißes Kloster, Sohag (Boud'hors) || Pergament, 2 Fragm. || 1 Kol. (*2) || 4-7 Zeilen (ca. *33-35) || 6,54-55.61-62; 15,4

647 10. Jh. (Schüssler) || Weißes Kloster, Sohag (Amélinaeu) || Pergament, 1 Fol., fragm. || 1 Kol. || 16-17 (*ca. 22) || 10,*24.25-31.33-37 || |ा bei 10,25

649 Amulett (BC: Osterlesung?) || 6. Jh. (Schüssler) || Herkunft unbekannt || Papyrus, 1 Fragm. || 1 Kol. (nach BC *2) || 17 Zeilen (laut BC *34) || Angaben nach BC unglaubhaft, da Faltungsspuren, doch keine weiteren Schriftspuren sichtbar sind. || 20,3-5 
663 ${ }^{\mathrm{L}}$ Typikon || 10.-12. Jh. (Askeland) || Weißes Kloster, Sohag (Louis) || Papier, 4 Fol. || 1 Kol. || 8-14 Zeilen || 12,24; 15,15; 19,1.16; 20,19; 21,12 (Incipits: 12,24; 15,15; 19,1; 20,19; 21,12; Versincipit als Desinit der Perikope 19,1-16: 19,16)

Für folgende Stücke war bei Drucklegung keine SMR-Nummer vorhanden bzw. lag keine Zuordnung zu einer bekannten Handschriftenzusammenstellung vor:

CGC 8086 [= Kairo, Koptisches Museum, CM 3770 (654); G 338b] || Datierung unbekannt || Herkunft unbekannt || Pergament, 1 Fragm. || 2 Kol. || ca. 12-15 Zeilen (*?) || 2,12-13.15. 17-18.*19.20-22 || Crum 1902: 27 No. 8086. || Ini. bei 2,13, 2,18, 2,21, 2,22

No. 931 [= Kairo, Koptisches Museum, Call-No. 931; G 809 Fragm. 1] || Datierung unbekannt || Herkunft unbekannt || Pergament, 1 Fragm. || 2 Kol. || 6-12 Zeilen (*?) || 16,*27. $28-29$. 32; $17,1.5-6||$ Ini. bei 16,29 und $* 17,4$

No. 948 [= Kairo, Koptisches Museum, Call-No. 948; G 805, Fragm. 2] || Datierung unbekannt || Herkunft unbekannt || Pergament, 1 Fragm. || 2 Kol. || 5-9 Zeilen (*?) || 19,25-26.*31. *36.*37.41-20,1 || Ini. bei *20,1, Glosse (c)d пाмג) bei 19,41 (bezogen auf das Versende als Desinit)

Drei Kodixfragmente sind Neufunde aus Deir el-Bachit, deren kodikologische Rekonstruktion noch nicht abgeschlossen ist, sodass die Informationen nur vorläufig sind:

P.Bachit $161+164$ Datierung unbekannt || Deir el-Bachit || Papyrus, 1 Fol. aus ca. 20 Fragm. || 1 Kol. || noch 8 Zeilen (*?) || 11, *53.54-55. *56.57-12,2

P.Bachit 255 Datierung unbekannt || Deir el-Bachit || Papyrus, 1 Fragm. || 1 Kol. || noch 10 Zeilen (*?) || 9,19-20.22

P.Bachit 357a (part.) Datierung unbekannt || Deir el-Bachit || Papyrus, 1 Fragm. || 1 Kol. || noch 9 Zeilen (*?) || 1,2-4 


\section{A1.1. Aufschlüsselung der Handschriften nach Material, Gattung sowie Datierung und Herkunft laut Literatur}

Für manche Informationen der Übersicht aufgenommener Handschriften erschien es sinnvoll, sie zum leichteren Zugang zu den benutzten Manuskripten und ihrem Text gegliedert zu wiederholen. Insbesondere hinsichtlich des Materials und der unterschiedlichen Gattungen einbezogener Handschriften sind für zukünftige Arbeiten Informationen zusammengestellt. Bei Provenienz und Datierungen folgen Angaben der in SMR-Datenbank und in Katalogen verzeichneten Literatur. Angaben zu heutigen Aufbewahrungsorten von Handschriften und ihren Teilen sind durch SMR-Datenbank und Listen bei Bedarf leicht zu ermitteln und werden hier daher nicht aufgeführt.

\section{Aufschlüsselung nach Material}

Der zur Vervielfältigung eines Textes genutzte Beschreibstoff sagt nichts über das Alter der enthaltenen Textversion aus, und nur bei Papier bietet er einen terminus post quem für die Herstellung einer Handschrift, da Papier, aus China herstammend, erst nach Mitte des 8. Jh. durch die Araber in Ägypten eingeführt wurde. ${ }^{71}$ Stets aber gibt der Beschreibstoff Anhaltspunkte für die relative Materialverfügbarkeit und zum finanziellen Aufwand, der mit einer Vervielfältigung einherging. Natürlich variieren Materialqualität und kalligraphische Qualität der Schrift, doch sind die Kosten bei Pergament wegen der aufwändigeren Herstellungsweise höher anzusetzen als bei Papyrus oder Papier. Die im Verhältnis zu anderen Schreibmaterialien sehr hohe Anzahl an Pergamentkodizes ist somit als Indiz zu werten, dass zu der Zeit, in der das Johannesevangelium im sahidischen Dialekt vervielfältigt wurde, Pergament trotz höherer Kosten das bevorzugte Schreibmaterial darstellte. ${ }^{72}$

71 Nach islamischer Tradition stehe die Einführung und sukzessive Vermittlung in den Mittelmeerraum und nach Europa mit der Schlacht am Fluss Talas (Kasachstan) 751 A.D. (133 A.H.) in Verbindung, bei der chinesische Papiermacher in arabische Gefangenschaft geraten seien. Tatsächlich war Papier in Persien zuvor bekannt. Unter Kalif Hārūn ar-Rašīd (†809) wurde es alleiniges Schreibmaterial offizieller Dokumente. Ab dem 9. Jh. sind Papiermühlen in Kairo belegt, ab dem 10. Jh. begann Papier, Papyrus als Beschreibstoff zu verdrängen. Papyrus und Pergament wurden seit vorchristlicher Zeit verwendet, Papyrus im islamischen Einflussgebiet bis ca. ins 14. Jh. und Pergament bis ins 14. bzw. 16. Jh., zieht man Spätdatierungen sahidischer Johanneshandschriften heran (112, 306 $\left.{ }^{\mathrm{L}}, 411^{\mathrm{L}}\right)$, siehe Gacek 2009: 186, 193-196; Lewis 1974: 91-93; O’Callaghan 1964: 10-11; Reed 1972: 86-116.

72 Sicher wäre es fahrlässig, zu weit reichende Schlüsse zu ziehen, da die Überlieferungslage nicht ideal ist, doch ist das unverhältnismäßige quantitative Überwiegen von Pergament be- 
Papier

Papyrus

Pergament

$16^{\mathrm{L}}|| 19|| 58^{\mathrm{L}}|| 59^{\mathrm{L}}|| 285|| 291^{\mathrm{L}}|| 292^{\mathrm{L}}|| 294^{\mathrm{L}}|| 349^{\mathrm{L}}|| 440^{\mathrm{L}}|| 663^{\mathrm{L}}$

ly 1 || ly 2 || pbo || 7 || 8 || 12 || 13 || 22 || 66 || 64 || 74 || 82 || 164 || 164 || 184 || 243 ||

|| 246 || 250 || 313 $2330^{\mathrm{L}}$ || 370 || 453 || 649 || P.Bachit $161+164$ || P.Bachit 255 || P.Bachit 357a (part.)

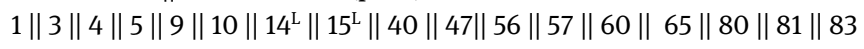

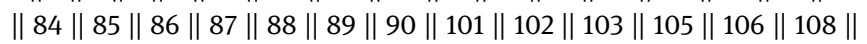

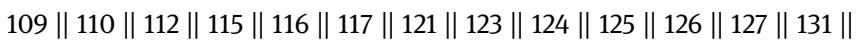

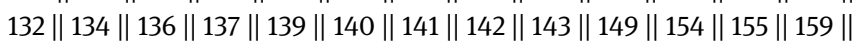

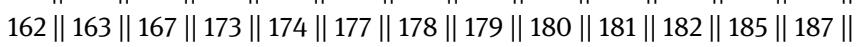

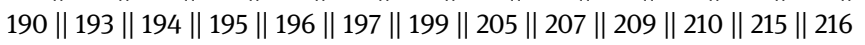
|| 217 || 218 || 266

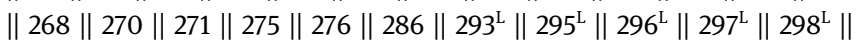
$299^{\mathrm{L}}|| 300^{\mathrm{L}}|| 301^{\mathrm{L}}|| 302^{\mathrm{L}}|| 305^{\mathrm{L}}|| 306^{\mathrm{L}}|| 308^{\mathrm{L}}|| 310^{\mathrm{L}}|| 317^{\mathrm{L}}|| 318^{\mathrm{L}}|| 324^{\mathrm{L}}||$

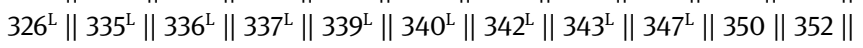

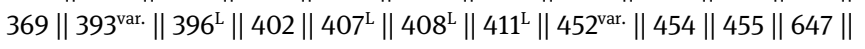
CGC 8086 || No. 931 || No. 948

\section{Aufschlüsselung nach Gattung}

Die intendierte Funktion bzw. Bestimmung eines Manuskripts definieren die Gattung, der es angehört. Bei liturgischen Kodizes wie Lektionaren ist die Funktion evident. In anderen Fällen, so bei als Amulett, Exzerpt oder Privatabschrift angesprochenen semiliterarischen Handschriften, ist die Behauptung einer Gattung bzw. Handschriftenart lediglich interpretativ. Bei Zitaten ist sie ohne Kenntnis des Textes, in den sie eingebettet sind, weitgehend unbrauchbar. Für andere, vordergründig leicht einer Gattung zuweisbare Manuskripte wie Volltexthandschriften ist sie bei näherer Untersuchung als Einordnungskriterium nur ein vorläufiger Behelf, der eine weit differenziertere Unterscheidung für jeden Einzelfall einfordert. Ansatzweise bieten paratextuelle Elemente wie Initialsetzungen, Glossen oder textstrukturierende Zeichen (Paragraphoi, Diplen, Koroniden und Obeloi) Hinweise, wie Einzelhandschriften zu werten sind. An späterer Stelle dieser Publikation werden genau diese Aspekte behandelt. Andere unterscheidende Merkmale wie Zwei- oder Mehrsprachigkeit und Wiederbe-

\footnotetext{
achtenswert. Es ist nicht damit zu erklären, dass Papier auf eine generell späte Entstehung hindeutet - vielleicht zu einer Zeit, in der Sahidisch kaum mehr Bedeutung besaß. Denkbar wäre, vor allem im Verhältnis zur Überlieferungslage bohairischer Handschriften, dass in Oberägypten Papier bis ins 13./14. Jh. noch keine sonderliche Verbreitung genoss, was unwahrscheinlich ist, oder Pergament hier ein spezifisch christlicher Beschreibstoff war. Das mag darauf hindeuten, dass die Pergamentproduktion im Umfeld von Klöstern tatsächlich wirtschaftlich sinnvoller war als der Ankauf von Papier oder die Nutzung von Papier bewusst vermieden wurde in Abgrenzung zum zunehmend islamischen Umfeld. Eine wissenschaftliche Untersuchung wäre sehr wünschenswert.
} 
schriftung (Palimpseste) sind zwar keine Gattungen an sich, doch ebenfalls geeignet, eine feinere Gliederung vorzunehmen. Miniatur-Kodizes und eine Handschrift mit Bibelorakel ( $\left.\dot{\varepsilon} \rho \eta \eta \varepsilon \varepsilon^{\alpha} \alpha\right)$ seien ebenfalls separat genannt. ${ }^{73}$

\section{Volltexthandschriften}

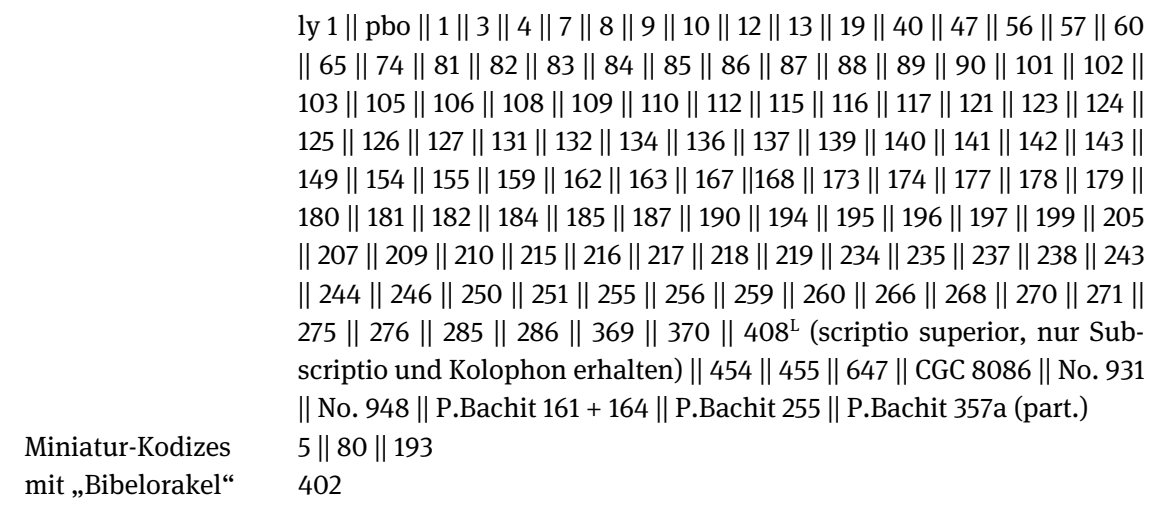

\section{liturgische Handschriften}

Lektionare

Evangelistare Pascha-Lektionare Sabbato-Kyriakai Typika Varia
$15^{\mathrm{L}}|| 58^{\mathrm{L}}||-59^{\mathrm{L}}$ (Sakramentale) || $295^{\mathrm{L}}$ (Marien- und Hochfeste) || $296^{\mathrm{L}}$ || $300^{\mathrm{L}}|| 302^{\mathrm{L}}|| 305^{\mathrm{L}}|| 306^{\mathrm{L}}|| 308^{\mathrm{L}}|| 310^{\mathrm{L}}|| 313^{\mathrm{L}}|| 317^{\mathrm{L}}|| 318^{\mathrm{L}}|| 324^{\mathrm{L}}|| 326^{\mathrm{L}} \|$ $330^{\mathrm{L}} \| 336^{\mathrm{L}}$ (Psalmtexte als griechisch-sahidische Bilingue) || $337^{\mathrm{L}}$ || 340 ${ }^{\mathrm{L}}$ || 350 (Bestimmung nicht gänzlich sicher) || 352 (Bestimmung nicht gänzlich sicher) $\left\|407^{\mathrm{L}}\right\| 408^{\mathrm{L}}$ (scriptio inferior) $14^{\mathrm{L}}|| 297^{\mathrm{L}}|| 335^{\mathrm{L}}|| 339^{\mathrm{L}}|| 343^{\mathrm{L}}|| 347^{\mathrm{L}}$

$16^{\mathrm{L}}|| 292^{\mathrm{L}}|| 298^{\mathrm{L}}|| 299^{\mathrm{L}}|| 349^{\mathrm{L}}$

$291^{\mathrm{L}}|| 293^{\mathrm{L}}$ (und Hochfeste) || $294^{\mathrm{L}}||$ | $301^{\mathrm{L}}$ (und Festtage)

$396^{\mathrm{L}} \| 663^{\mathrm{L}}$

$342^{\mathrm{L}}$ (bilingue liturgische Handschrift) $\| 440^{\mathrm{L}}$ (polyglotte Handschrift/ Processionale zum Wüstenfest des Apa Schenute)

\section{Zitate (in einem literarischen Text)}

Zitate

453 (in einer Homilie?)

\section{semiliterarische Handschriften}

Amulette

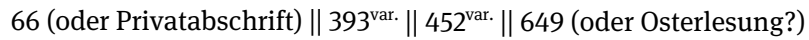

Privatabschriften

Exzerpt

liturgisch

Textauszug
22

$411^{\mathrm{L}}$

ly 2

$73 \mathrm{Zu} \dot{\varepsilon} \rho \mu \eta v \varepsilon i ́ \alpha$ in biblischen Manuskripten siehe Cirafesi 2014. Der Begriff wird im Koptischen auch bei Typika und bohairischen Katenenhandschriften verwendet. 


\section{mehrsprachige Handschriften}

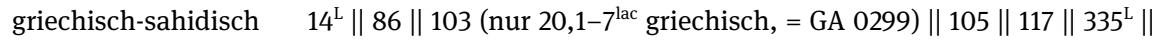

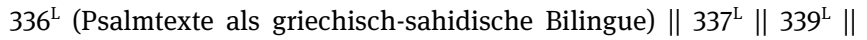
$340^{\mathrm{L}}|| 342^{\mathrm{L}}|| 343^{\mathrm{L}}|| 347^{\mathrm{L}}|| 350|| 352$

sahidisch-arabisch $\quad 16^{\mathrm{L}} \| 349^{\mathrm{L}}$

polyglott $\quad 440^{\mathrm{L}}$ (griechisch-sahidisch-bohairisch-arabisch)

\section{Palimpseste}

J als scriptio inferior $\quad 219 \| 408^{\mathrm{L}}$ (als Perikope des Lektionars)

J als scriptio superior $\quad 89|| 142|| 182$ || 185 || $396^{\mathrm{L}}$ || $408^{\mathrm{L}}$ (nur Subscriptio und Kolophon erhalten)

\section{Aufschlüsselung nach Herkunft laut Angaben der Literatur}

Nur selten stammen Handschriften aus gesicherten (archäologischen) Kontexten. Zur Bestimmung der Herkunft ist man zumeist auf Informationen von Kunsthändlern angewiesen, die als Vermittler zwischen Findern und ankaufenden Bibliotheken, Sammlern oder Wissenschaftlern fungieren. Wenn ein paläographischer Vergleich die Zuweisung von über verschiedene Sammlungen verstreuten Folien und Fragmenten zueinander erlaubt, können als Ausgangspunkt zur Ermittlung der Herkunft auch Bibliothekssignaturen dienen, für die frühere Bearbeiter eine gesicherte Provenienz verzeichnen. ${ }^{74}$ Im Folgenden werden Provenienzangaben der Literatur, auch nur vermutete, mit den zugewiesenen Handschriften gelistet. Für einige mit unbekannter Herkunft geführte Manuskripte dürfte sich ebenso eine Provenienz ermitteln lassen, doch ist eine solche Recherche nicht Aufgabe einer kritischen Textedition, sondern durch Einzeluntersuchungen oder Handschriftenkataloge zu leisten.

\section{Herkunft nach Angaben der Literatur}

Deir Abu Maqar

Deir el-Bachit

Deir el-Bala'izah
286

P.Bachit $161+164$ || P.Bachit 255 || P.Bachit 357a (part.)

177 || 207

74 Insbesondere für Folien oder Fragmente der Signaturgruppen Copte 129,1-133,2 der französischen Nationalbibliothek lässt sich wegen ihrer Erwerbungsgeschichte recht sicher eine Herkunft aus dem Weißen Kloster, Sohag, behaupten. Bei diesen Manuskripten wurde in der Übersicht aufgenommener Handschriften auf É. Amélineau verwiesen, der 1888/1889 das handschriftliche Inventarbuch dieser Signaturgruppen erstellte („Catalogue des Manuscrits Coptes Provenant du Acquisitions 7806, 7892, 8050 \& du Don 2616“). Mit gleichem Recht ließen sich G. Maspero, der den Erwerb initiierte, oder der französische Antiquitätenhändler A. Frènay nennen, den Gewährsmann für eine Herkunft aus dem Weißen Kloster, siehe Louis 2005. Siehe aber BC 1.1: 50 sa 8, wonach R. Curzon 1838 Komplementblätter (London, BL, Or. 8810 f. 1-6) zu Folien in Paris in Deir Amba Bschoi im Wadi Natrun ankaufte. 
Deir el-Naqlun

19|| 65

„wohl Dishna“

pbo $\| 1$

el-Aschmunein

81|| 193

Epiphanius-Kloster, Theben

$407^{\mathrm{L}}$

„wohl Faijum“

259

Michaels-Kloster, Harabt Hamuli

Jeremiaskloster, Saqqara

$9\|10\| 15^{\mathrm{L}}$

„eher Nordägypten“

Theben

4 || 5

Touton (als Sciptorium)

$59^{\mathrm{L}}$ (Schüssler, siehe auch „Weißes Kloster, Sohag“)

7

Wadi Sarga

Weißes Kloster, Sohag

85 ?

163 || 219 || 271

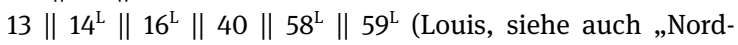
ägypten“) || 101 || 102 || 103 || 105 || 112

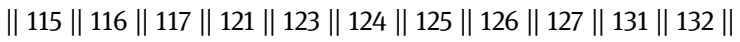

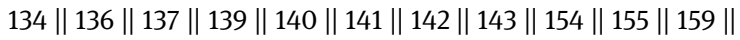

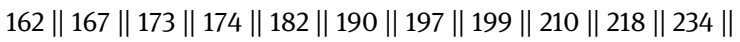

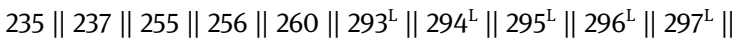
$298^{\mathrm{L}}|| 299^{\mathrm{L}}|| 300^{\mathrm{L}}|| 301^{\mathrm{L}}|| 302^{\mathrm{L}}|| 305^{\mathrm{L}}|| 306^{\mathrm{L}}|| 310^{\mathrm{L}}|| 317^{\mathrm{L}} \|$ $324^{\mathrm{L}}$ || $326^{\mathrm{L}}$ || $335^{\mathrm{L}}$ || $336^{\mathrm{L}}$ || $337^{\mathrm{L}}$ || $339^{\mathrm{L}}$ || $340^{\mathrm{L}}$ || $342^{\mathrm{L}}$ || $347^{\mathrm{L}}$ || $349^{\mathrm{L}}|| 352$ || 369 || $440^{\mathrm{L}}|| 455$ || 647 || $663^{\mathrm{L}}$

Herkunft unbekannt

ly 1 || ly 2 || 3 || 8 || 12 || 22 || 47 || 56 || 57 || 60 || 66 || 74 || 80 || 82

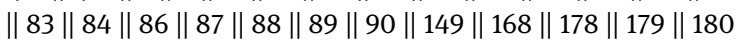
|| 181 || 184 || 185 || 187 || 194 || 195 || 196 || 205 || 209 || 216 || 216 || 217 || 238 || 243 || 245 || 276 || 285 || 291 || 292 || $308^{\mathrm{L}}|| 313^{\mathrm{L}}|| 318^{\mathrm{L}}|| 330^{\mathrm{L}}|| 343^{\mathrm{L}}|| 350$

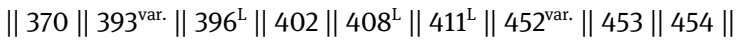
649 || CGC 8086 || No. 931 || No. 948

\section{Aufschlüsselung nach Datierungen laut Angaben der Literatur}

Datierungen koptischer Handschriften sind derjenige Bereich, der am wenigsten beanspruchen kann, in irgendeiner Weise genau oder mehr als nur vorläufig zu sein. Zwei Gruppen von Handschriften lassen sich vorab aus der weiteren Behandlung ausschließen, zunächst Handschriften, für die laut den Literaturangaben der SMR-Datenbank keine Datierungen geboten werden, sodann Handschriften, die durch ihre Kolophone absolut datiert sind:

\section{Datierung unbekannt}

ly 2 || 84 || 85 || 86 || 87 || 88 || 89 || 90 || 149 || 184 || 185 || 266 || 326 $330^{\mathrm{L}}||$ 352 || $396^{\mathrm{L}}$ || $407^{\mathrm{L}}$ || $408^{\mathrm{L}}$ || 454 || 455 || CGC 8086 || No. 931 || No. 948 || P.Bachit 161 + 164 || P.Bachit 255 || P.Bachit 357a (part.)

\section{exakte Datierungen durch Kolophone (nach julianischem Kalender)}

29.08.861-28.08.862 10

29.08.876-28.08.877 65 
17.11.1099-06.11.1100 19

29.08.1117-28.08.1118 $305^{\mathrm{L}}$

Wenn nicht Kolophone eine gesicherte Datierung liefern, ist man auf Vorschläge der Literatur angewiesen, die für verschiedene Teile einer Handschrift auch beim selben Autor teils massiv divergieren können. ${ }^{75}$ So ist es nicht verwunderlich, dass Vorschläge zur zeitlichen Einordnung bei verschiedenen Autoren ebenfalls kaum einen Konsens erkennen lassen. ${ }^{76} \mathrm{Um}$ zu ermöglichen, für die sahidischen Johanneshandschriften wenigstens die frühesten und spätesten Datierungen der Literatur vergleichen zu können, werden sie nacheinander in Tabellen geboten. Sind früheste und späteste Datierung identisch, bedeutet das gewöhnlich, dass lediglich eine Sekundärpublikation einen Datierungsvorschlag bietet. Es ist kein Hinweis auf einen Forschungskonsens.

\section{früheste Datierungen nach Angaben der Literatur}

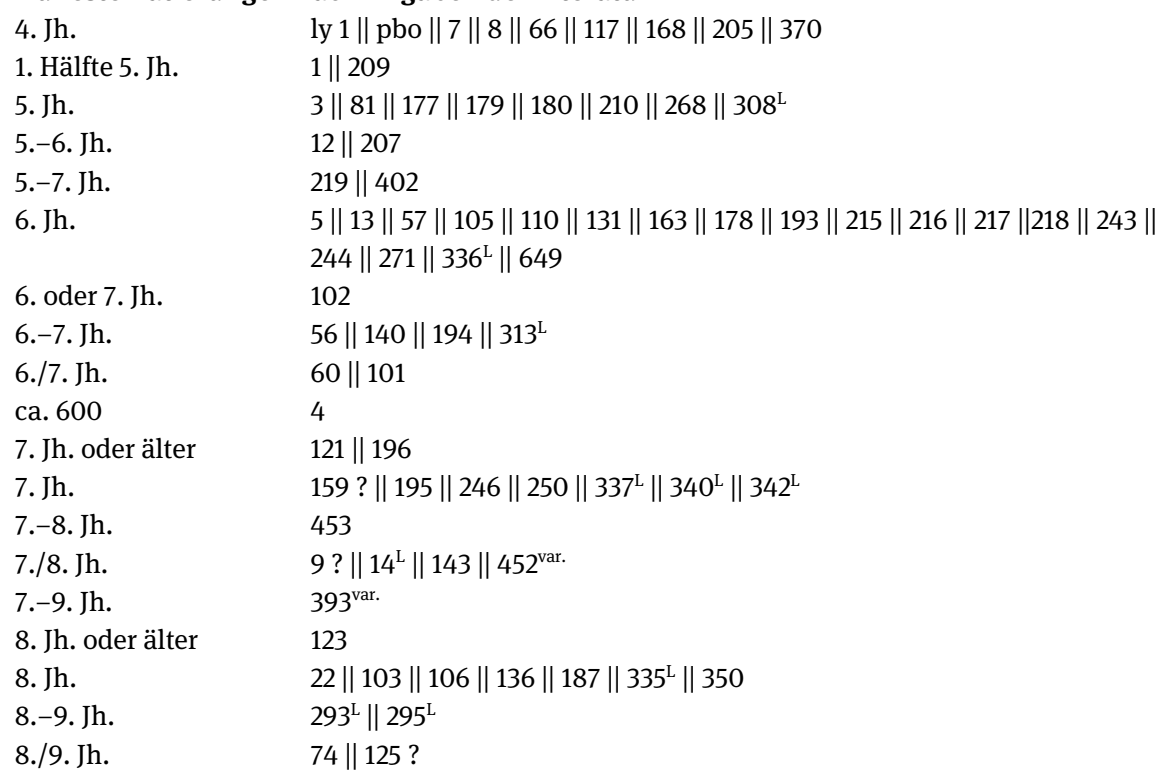

75 Siehe $14^{\mathrm{L}}$ (Clark), 112 (Horner), 117 (Hyvernat), 123 (Horner), 140 (Horner), 143 (Horner), 181 (Horner), 298 (Till), 299 (Till), 335 (Aland, Horner und van Haelst für verschiedene Teile), $340^{\mathrm{L}}$ (Aland) sowie als besonders eindrückliche Beispiele 121 (Horner für verschiedene Teile um über 500 Jahre), $337^{\mathrm{L}}$ (Aland für verschiedene Teile um 500 Jahre) und $342^{\mathrm{L}}$ (Aland für verschiedene Teile um 700 Jahre und van Haelst für verschiedene Teile um 600 Jahre).

76 Um gut 500, teils sogar 800 Jahre weichen Früh- und Spätdatierungsvorschläge bei $14^{\mathrm{L}}$, $103,110,117,121,131,140,142,294^{\mathrm{L}}, 295^{\mathrm{L}}, 296^{\mathrm{L}}, 299^{\mathrm{L}}, 300^{\mathrm{L}}, 306^{\mathrm{L}}$ und $336^{\mathrm{L}}$ voneinander ab. 


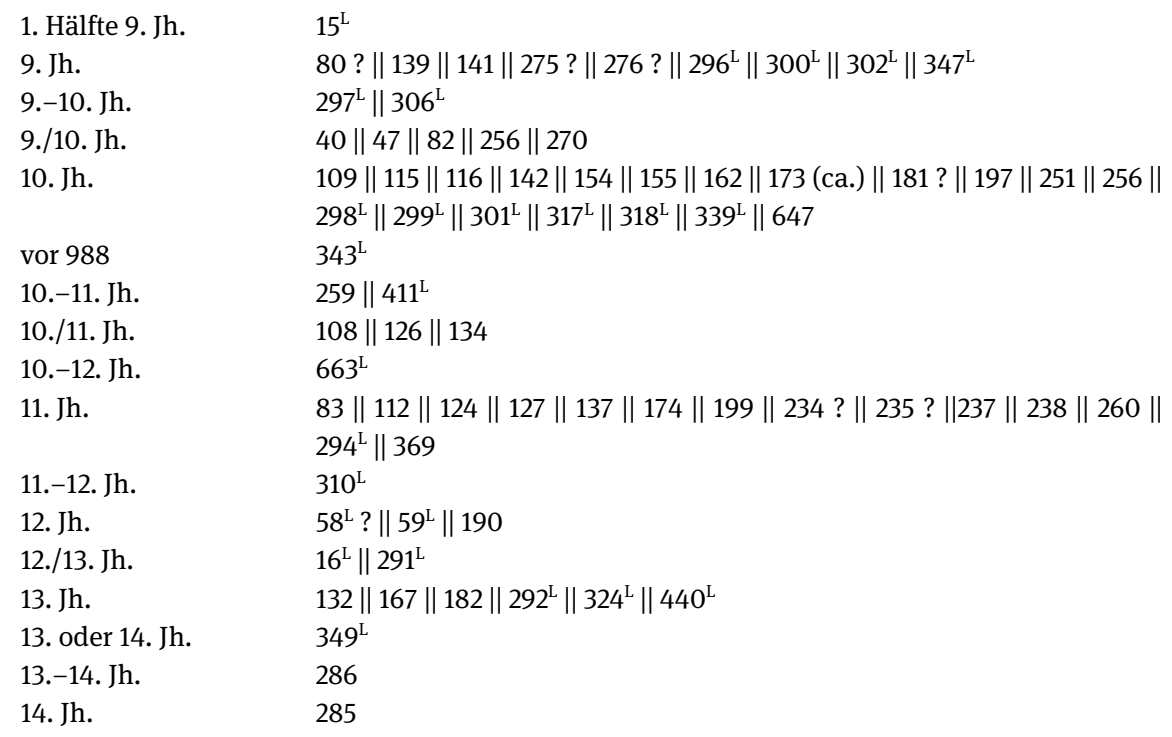

\section{späteste Datierungen nach Angaben der Literatur}

4. Jh.

1. Hälfte 5. Jh.

5. Jh.

5.-6. Jh.

5. -7 . Jh.

6. Jh.

6. -7. Jh.

6./7. Jh.

6. -8 . Jh.

7. Jh. oder älter

7. Jh.

7.-8. Jh.

7./8. Jh.

7.-9. Jh.

8. Jh.

8. -9. Jh.

8./9. Jh.

8. oder 9. Jh.

frühes 9. Jh.

9. Jh.

9.-10. Jh.

9./10. Jh.

10. Jh.

vor 988

10.-11. Jh. ly 1 || pbo || 66 || 168 || 205 || 370

209

1 || 81 || 180 || 210 || 268 || 308

12

219|| 402

57 || 163 || 179 (са.) || 193 || 207 || 215 || 217 || 218 || 243 || 244 || 271 || 649

56 || 178 || 194

60

3

196|| 216

7 || 8 || 159 ? || 177 || 195 || 246 || 250 || 313

453

$452^{\text {var. }}$

$393^{\text {var. }}$

22 || 101 || 350

13

187

102

74

4 ? || 5 ? || $15^{\mathrm{L}}||$ | 80 ? || 90 ? || 105 || 106 || 136 || 275 |

$9 \| 131$

40 || 47 || 82|| $\mid 255$ || 270

109 || 115 || 117 || 123 || 140 || 154 || 162 || 173 || 197 || 251 || 256 || 297 $29317^{\mathrm{L}}$ || $318^{\mathrm{L}}|| 335^{\mathrm{L}}\left\|347^{\mathrm{L}}\right\| 647$

$343^{\mathrm{L}}$

259 


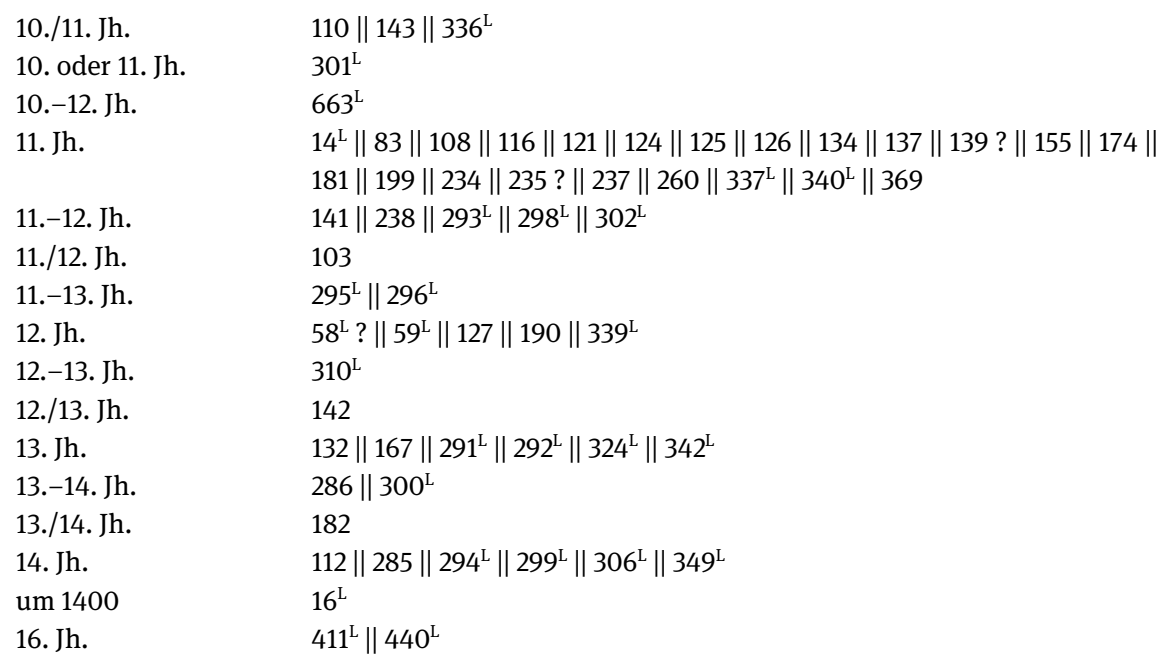

Abschließend seien alle Handschriften unter Angabe des Beschreibstoffes gelistet. Sind Datierungsvorschläge vorhanden, werden früheste und späteste Datierung nebeneinander gestellt. Erneut sei darauf hingewiesen, dass identische früheste und späteste Datierung keinen Forschungskonsens, sondern fast immer einen singulären Datierungsvorschlag bedeuten.

\begin{tabular}{llll}
\hline & früheste Datierung & späteste Datierung & Material \\
\hline ly 1 & 4. Jh. & 4. Jh. & Papyrus \\
ly 2 & Datierung unbekannt & Datierung unbekannt & Papyrus \\
pbo & 4. Jh. & 4. Jh. & Papyrus \\
1 & 1. Hälfte 5. Jh. & 5. Jh. & Pergament \\
3 & 5. Jh. & 6.-8. Jh. & Pergament \\
4 & ca. 600 & 9. Jh. ? & Pergament \\
5 & 6. Jh. & 9. Jh. ? & Pergament \\
7 & 4. Jh. & 7. Jh. & Papyrus \\
8 & 4. Jh. & 7. Jh. & Papyrus \\
9 & 7./8. Jh. ? & 9.- 10. Jh. & Pergament \\
10 & 29.08.861-28.08.862 & & Pergament \\
12 & 5.-6. Jh. & 5.-6. Jh. & Papyrus \\
13 & 6. Jh. & 8.-9. Jh. & Papyrus \\
$14^{\mathrm{L}}$ & 7./8. Jh. & 11. Jh. & Pergament \\
$15^{\mathrm{L}}$ & 1. Hälfte 9. Jh. & 9. Jh. & Pergament \\
$16^{\mathrm{L}}$ & 12./13. Jh. & um 1400 & Papier \\
19 & 17.11.1099-06.11.1100 & & Papier \\
22 & 8. Jh. & 8. Jh. & Papyrus \\
40 & 9./10. Jh. & 9./10. Jh. & Pergament \\
47 & 9./10. Jh. & 9./10. Jh. & Pergament \\
56 & 6.-7. Jh. & 6.-7. Jh. & Pergament \\
& & &
\end{tabular}




\begin{tabular}{|c|c|c|c|}
\hline & früheste Datierung & späteste Datierung & Material \\
\hline 57 & 6. Jh. & 6. Jh. & Pergament \\
\hline $58^{\mathrm{L}}$ & 12. Jh.? & 12. Jh.? & Papier \\
\hline $59^{\mathrm{L}}$ & 12. Jh. & 12. Jh. & Papier \\
\hline 60 & 6./7. Jh. & 6./7. Jh. & Pergament \\
\hline 65 & 29.08.876-28.08.877 & & Pergament \\
\hline 66 & 4. Jh. & 4. Jh. & Papyrus \\
\hline 74 & 8./9. Jh. & frühes 9. Jh. & Papyrus \\
\hline 80 & 9. Jh.? & 9. Jh. ? & Pergament \\
\hline 81 & 5. Jh. & 5. Jh. & Pergament \\
\hline 82 & 9./10. Jh. & 9./10. Jh. & Papyrus \\
\hline 83 & 11. Jh. & 11. Jh. & Pergament \\
\hline 84 & Datierung unbekannt & Datierung unbekannt & Pergament \\
\hline 85 & Datierung unbekannt & Datierung unbekannt & Pergament \\
\hline 86 & Datierung unbekannt & Datierung unbekannt & Pergament \\
\hline 87 & Datierung unbekannt & Datierung unbekannt & Pergament \\
\hline 88 & Datierung unbekannt & Datierung unbekannt & Pergament \\
\hline 89 & Datierung unbekannt & Datierung unbekannt & Pergament \\
\hline 90 & Datierung unbekannt & Datierung unbekannt & Pergament \\
\hline 101 & 6./7. Jh. & 8. Jh. & Pergament \\
\hline 102 & 6. oder 7. Jh. & 8. oder 9. Jh. & Pergament \\
\hline 103 & 8. Jh. & 11./12. Jh. & Pergament \\
\hline 105 & 6. Jh. & 9. Jh. & Pergament \\
\hline 106 & 8. Jh. & 9. Jh. & Pergament \\
\hline 108 & 10./11. Jh. & 11. Jh. & Pergament \\
\hline 109 & 10. Jh. & 10. Jh. & Pergament \\
\hline 110 & 6. Jh. & 10./11. Jh. & Pergament \\
\hline 112 & 11. Jh. & 14. Jh. & Pergament \\
\hline 115 & 10. Jh. & 10. Jh. & Pergament \\
\hline 116 & 10. Jh. & 11. Jh. & Pergament \\
\hline 117 & 4. Jh. & 10. Jh. & Pergament \\
\hline 121 & 7. Jh. & 10./11. Jh. & Pergament \\
\hline 123 & 8. Jh. oder älter & 10. Jh. & Pergament \\
\hline 124 & 11. Jh. & 11. Jh. & Pergament \\
\hline 125 & 8./9. Jh.? & 11. Jh. & Pergament \\
\hline 126 & 10./11. Jh. & 11. Jh. & Pergament \\
\hline 127 & 11. Jh. & 12. Jh. & Pergament \\
\hline 131 & 6. Jh. & 9.-10. Jh. & Pergament \\
\hline 132 & 13. Jh. & 13. Jh. & Pergament \\
\hline 134 & 10./11. Jh. & 11. Jh. & Pergament \\
\hline 136 & 8. Jh. & 9. Jh. & Pergament \\
\hline 137 & 11. Jh. & 11. Jh. & Pergament \\
\hline 139 & 9. Jh. & 11. Jh.? & Pergament \\
\hline 140 & 6.-7. Jh. & 10. Jh. & Pergament \\
\hline 141 & 9. Jh. & 11.-12. Jh. & Pergament \\
\hline 142 & 10. Jh. & 12./13. Jh. & Pergament \\
\hline 143 & 7./8. Jh. & 10./11. Jh. & Pergament \\
\hline
\end{tabular}




\begin{tabular}{|c|c|c|c|}
\hline & früheste Datierung & späteste Datierung & Material \\
\hline 149 & Datierung unbekannt & Datierung unbekannt & Pergament \\
\hline 154 & 10. Jh. & 10. Jh. & Pergament \\
\hline 155 & 10. Jh. & 11. Jh. & Pergament \\
\hline 159 & 7. Jh.? & 7. Jh.? & Pergament \\
\hline 162 & 10. Jh. & 10. Jh. & Pergament \\
\hline 163 & 6. Jh. & 6. Jh. & Pergament \\
\hline 167 & 13. Jh. & 13. Jh. & Pergament \\
\hline 168 & 4. Jh. & 4. Jh. & Papyrus \\
\hline 173 & ca. 10. Jh. & 10. Jh. & Pergament \\
\hline 174 & 11. Jh. & 11. Jh. & Pergament \\
\hline 177 & 5. Jh. & 7. Jh. & Pergament \\
\hline 178 & 6. Jh. & 6.-7. Jh. & Pergament \\
\hline 179 & 5. Jh. & ca. 6. Jh. & Pergament \\
\hline 180 & 5. Jh. & 5. Jh. & Pergament \\
\hline 181 & 10. Jh. ? & 11. Jh. & Pergament \\
\hline 182 & 13. Jh. & 13./14. Jh. & Pergament \\
\hline 184 & Datierung unbekannt & Datierung unbekannt & Papyrus \\
\hline 185 & Datierung unbekannt & Datierung unbekannt & Pergament \\
\hline 187 & 8. Jh. & 8./9. Jh. & Pergament \\
\hline 190 & 12. Jh. & 12. Jh. & Pergament \\
\hline 193 & 6. Jh. & 6. Jh. & Pergament \\
\hline 194 & 6.-7. Jh. & 6. $-7 . \mathrm{Jh}$. & Pergament \\
\hline 195 & 7. Jh. & 7. Jh. & Pergament \\
\hline 196 & 7. Jh. oder älter & 7. Jh. oder älter & Pergament \\
\hline 197 & 10. Jh. & 10. Jh. & Pergament \\
\hline 199 & 11. Jh. & 11. Jh. & Pergament \\
\hline 205 & 4. Jh. & 4. Jh. & Pergament \\
\hline 207 & 5.-6. Jh. & 6. Jh. & Pergament \\
\hline 209 & 1. Hälfte 5. Jh. & 1. Hälfte 5. Jh. & Pergament \\
\hline 210 & 5. Jh. & 5. Jh. & Pergament \\
\hline 215 & 6. Jh. & 6. Jh. & Pergament \\
\hline 216 & 6. Jh. & 7. Jh. oder älter & Pergament \\
\hline 217 & 6. Jh. & 6. Jh. & Pergament \\
\hline 218 & 6. Jh. & 6. Jh. & Pergament \\
\hline 219 & 5.-7. Jh. & 5. -7. Jh. & Pergament \\
\hline 234 & 11. Jh. ? & 11. Jh. & Pergament \\
\hline 235 & 11. Jh. ? & 11. Jh. ? & Pergament \\
\hline 237 & 11. Jh. & 11. Jh. & Pergament \\
\hline 238 & 11. Jh. & 11.-12. Jh. & Pergament \\
\hline 243 & 6. Jh. & 6. Jh. & Papyrus \\
\hline 244 & 6. Jh. & 6. Jh. & Papyrus \\
\hline 246 & 7. Jh. & 7. Jh. & Papyrus \\
\hline 250 & 7. Jh. & 7. Jh. & Papyrus \\
\hline 251 & 10. Jh. & 10. Jh. & Pergament \\
\hline 255 & 9./10. Jh. & 9./10. Jh. & Pergament \\
\hline 256 & 10. Jh. & 10. Jh. & Pergament \\
\hline
\end{tabular}




\begin{tabular}{|c|c|c|c|}
\hline & früheste Datierung & späteste Datierung & Material \\
\hline 259 & 10.-11. Jh. & 10.-11. Jh. & Pergament \\
\hline 260 & 11. Jh. & 11. Jh. & Pergament \\
\hline 266 & Datierung unbekannt & Datierung unbekannt & Pergament \\
\hline 268 & 5. Jh. & 5. Jh. & Pergament \\
\hline 270 & 9./10. Jh. & 9./10. Jh. & Pergament \\
\hline 271 & 6. Jh. & 6. Jh. & Pergament \\
\hline 275 & 9. Jh.? & 9. Jh.? & Pergament \\
\hline 276 & 9. Jh.? & 9. Jh.? & Pergament \\
\hline 285 & 14. Jh. & 14. Jh. & Papier \\
\hline 286 & 13. -14 . Jh. & 13. -14 . Jh. & Pergament \\
\hline $291^{\mathrm{L}}$ & 12./13. Jh. & 13. Jh. & Papier \\
\hline $292^{\mathrm{L}}$ & 13. Jh. & 13. Jh. & Papier \\
\hline $293^{\mathrm{L}}$ & 8. $-9 . \mathrm{Jh}$. & 11. $-12 . J h$. & Pergament \\
\hline $294^{\mathrm{L}}$ & 11. Jh. & 14. Jh. & Papier \\
\hline $295^{\mathrm{L}}$ & 8. -9. Jh. & 11.-13. Jh. & Pergament \\
\hline $296^{\mathrm{L}}$ & 9. Jh. & 11.-13. Jh. & Pergament \\
\hline $297^{\mathrm{L}}$ & 9.-10. Jh. & 10. Jh. & Pergament \\
\hline $298^{\mathrm{L}}$ & 10. Jh. & 11.-12. Jh. & Pergament \\
\hline $299^{\mathrm{L}}$ & 10. Jh. & 14. Jh. & Pergament \\
\hline $300^{\mathrm{L}}$ & 9. Jh. & 13.-14. Jh. & Pergament \\
\hline $301^{\mathrm{L}}$ & 10. Jh. & 11. Jh. & Pergament \\
\hline $302^{\mathrm{L}}$ & 9. Jh. & 11. $-12 . J h$. & Pergament \\
\hline $305^{\mathrm{L}}$ & $29.08 .1117-28.08 .1118$ & & Pergament \\
\hline $306^{\mathrm{L}}$ & 9.-10. Jh. & 14. Jh. & Pergament \\
\hline $308^{\mathrm{L}}$ & 5. Jh. & 5. Jh. & Pergament \\
\hline $310^{\mathrm{L}}$ & 11.-12. Jh. & 12.-13. Jh. & Pergament \\
\hline $313^{\mathrm{L}}$ & 6.-7. Jh. & 7. Jh. & Papyrus \\
\hline $317^{\mathrm{L}}$ & 10. Jh. & 10. Jh. & Pergament \\
\hline $318^{\mathrm{L}}$ & 10. Jh. & 10. Jh. & Pergament \\
\hline $324^{\mathrm{L}}$ & 13. Jh. & 13. Jh. & Pergament \\
\hline $326^{\mathrm{L}}$ & Datierung unbekannt & Datierung unbekannt & Pergament \\
\hline $330^{\mathrm{L}}$ & Datierung unbekannt & Datierung unbekannt & Papyrus \\
\hline $335^{\mathrm{L}}$ & 8. Jh. & 10. Jh. & Pergament \\
\hline $336^{\mathrm{L}}$ & 6. Jh. & 10./11. Jh. & Pergament \\
\hline $337^{\mathrm{L}}$ & 7. Jh. & 11. Jh. & Pergament \\
\hline $339^{\mathrm{L}}$ & 10. Jh. & 12. Jh. & Pergament \\
\hline $340^{\mathrm{L}}$ & 7. Jh. & 11. Jh. & Pergament \\
\hline $342^{\mathrm{L}}$ & 7. Jh. & 13. Jh. & Pergament \\
\hline $343^{\mathrm{L}}$ & vor 988 & vor 988 & Pergament \\
\hline $347^{\mathrm{L}}$ & 9. Jh. & 10. Jh. & Pergament \\
\hline $349^{\mathrm{L}}$ & 13. Jh. & 14. Jh. & Papier \\
\hline 350 & 8. Jh. & 8. Jh. & Pergament \\
\hline 352 & Datierung unbekannt & Datierung unbekannt & Pergament \\
\hline 369 & 11. Jh. & 11. Jh. & Pergament \\
\hline 370 & 4. Jh. & 4. Jh. & Papyrus \\
\hline $393^{\text {var. }}$ & 7.-9. Jh. & 7.-9. Jh. & Pergament \\
\hline
\end{tabular}




\begin{tabular}{llll}
\hline & früheste Datierung & späteste Datierung & Material \\
\hline $396^{\mathrm{L}}$ & Datierung unbekannt & Datierung unbekannt & Pergament \\
402 & 5.-7. Jh. & 5.-7. Jh. & Pergament \\
$407^{\mathrm{L}}$ & Datierung unbekannt & Datierung unbekannt & Pergament \\
$408^{\mathrm{L}}$ & Datierung unbekannt & Datierung unbekannt & Pergament \\
$411^{\mathrm{L}}$ & 10.-11. Jh. & 16. Jh. & Pergament \\
$440^{\mathrm{L}}$ & 13. Jh. & 16. Jh. & Papier \\
$452^{\text {var. }}$ & 7./8. Jh. & 7./8. Jh. & Pergament \\
453 & 7.-8. Jh. & 7.-8. Jh. & Papyrus \\
454 & Datierung unbekannt & Datierung unbekannt & Pergament \\
455 & Datierung unbekannt & Datierung unbekannt & Pergament \\
647 & 10. Jh. & 10. Jh. & Pergament \\
649 & 6. Jh. & 6. Jh. & Papyrus \\
$663^{\mathrm{L}}$ & 10.-12. Jh. & 10.-12. Jh. & Papier \\
CGC 8086 & Datierung unbekannt & Datierung unbekannt & Pergament \\
No. 931 & Datierung unbekannt & Datierung unbekannt & Pergament \\
No. 948 & Datierung unbekannt & Datierung unbekannt & Pergament \\
P.Bachit 161 & Datierung unbekannt & Datierung unbekannt & Papyrus \\
164 & & & \\
P.Bachit 255 & Datierung unbekannt & Datierung unbekannt & Papyrus \\
P.Bachit 357a & Datierung unbekannt & Datierung unbekannt & Papyrus \\
(part.) & & &
\end{tabular}




\section{A1.2. Handschriftliche Bezeugung der Einzelverse}

Die Auflistung berücksichtigt nur sahidische Manuskripte. Ein Sternchen (*/Asteriskus) bezeichnet Verse, die nur Einzelbuchstaben oder sehr fragmentierte Wörter bieten oder unleserlich sind. Diese Handschriften wurden zwar gesichtet, erlauben im Normalfall aber keine Auswertung hinsichtlich Textvarianten oder orthographischer Besonderheiten.

Von 127 lag für Kairo, CM, CM 2710 [früher Kairo, Patr. (ohne Nr.)] nur ein Bild von Recto vor, sodass eine Kollation von 9,4-16 und Teilen von 9,3 und 9,17 nicht möglich war. ${ }^{77}$ Die entsprechenden Verse sind in geschweifte Klammern gesetzt. Wegen Fragmentierung ausgesondert und ebenfalls in geschweifte Klammern gesetzt wurde 47.

Inscriptio 9, 10, 15 (liturgische Rubrik), 19, *40, 47 (m2), 134, 293 (liturgische Rubrik), 295 (liturgische Rubrik), $\left\{340^{\mathrm{L}}\right.$ (liturgische Rubrik zur griechischen Perikope, daher im Apparat zum sahidischen Text ungenannt)\}, 411 ${ }^{\mathrm{L}}$ (liturgische Rubrik, Privatabschrift), $452^{\text {var. }}$ (Amulett)

$1,11,3,4,5,9,10,13,15^{\mathrm{L}}, 19,40,\{47\}, 90,106,134,293^{\mathrm{L}}, 295^{\mathrm{L}}, 393^{\mathrm{var}}$ (Amulett), 411 ${ }^{\mathrm{L}}$, $452^{\text {var. }}$ (Amulett), 479

$1,21,3,4,5,9,13,15^{\mathrm{L}}, 19,40,\{47\}, 90,106,134,139,293^{\mathrm{L}}, 295^{\mathrm{L}}, 411^{\mathrm{L}}$, P.Bachit 357a (part.)

$1,31,3,4,5,9,10,13,15^{\mathrm{L}}, 19,40,\{47\}, 90,106,134,139,293^{\mathrm{L}}, 295^{\mathrm{L}}, 411^{\mathrm{L}}$, P.Bachit $357 \mathrm{a}$ (part.)

$1,41,3,4,5,9,10,13,15^{\mathrm{L}}, 19,40,\{47\}, 90,106,134,139,181,293^{\mathrm{L}}, 295^{\mathrm{L}}, 411^{\mathrm{L}}$, P.Bachit 357 a (part.)

$1,511,3,4,5,9,10,13,15^{\mathrm{L}}, 19,\{47\}, 90,106,134,139,181,293^{\mathrm{L}}, 295^{\mathrm{L}}, 411^{\mathrm{L}}$

$1,61,3,4,5,9,10,13,15^{\mathrm{L}}, 19,40,\{47\}, 90,106,134,139,181,293^{\mathrm{L}}, 295^{\mathrm{L}}(2 \times), 396^{\mathrm{L}}$

$1,71,3,4,5,9,10,13,15^{\mathrm{L}}, 19, * 40,\{47\}, 103,117,134,139,181,293^{\mathrm{L}}, 295^{\mathrm{L}}(2 \times, 2$. Mal als $295^{\mathrm{L}}$ a verzeichnet)

$1,8 \quad 1,3,4,5,9,10,12,13,15^{\mathrm{L}}, 19,\{47\}, 117,134,139,181,293^{\mathrm{L}}, 295^{\mathrm{L}}(2 \times)$

$1,91,3,4,5,9,10,12,13,15^{\mathrm{L}}, 19,\{47\}, 117,134,139,181,293^{\mathrm{L}}, 295^{\mathrm{L}}(2 \times, 2$. Mal nur noүoєiN erhalten)

$1,101,3,4,5,9,10,13,14^{\mathrm{L}}, 15^{\mathrm{L}}, 19,\{47\}, 117,134,139,181,293^{\mathrm{L}}, 295^{\mathrm{L}}$

$1,111,3,4,5,9,10,13,14^{\mathrm{L}}, 15^{\mathrm{L}}, 19,\{47\}, 101,117,134,139,181,293^{\mathrm{L}}, 295^{\mathrm{L}}$

$1,121,3,4,5,9,10,12,13, * 14^{\mathrm{L}}, 15^{\mathrm{L}},\{47\}, 19,101,134,181,293^{\mathrm{L}}, 295^{\mathrm{L}}$

$1,131,3,4,5,9,10,12,13, * 14^{\mathrm{L}}, 15^{\mathrm{L}}, 19,\{47\}, 103,134,139,181,293^{\mathrm{L}}, 295^{\mathrm{L}}$

$1,141,3,4,5,9,10,12,13,14^{\mathrm{L}}, 15^{\mathrm{L}}, 19,\{47\}, 103,134,139,181,295^{\mathrm{L}}$

$1,15 \quad 1,3,4,5,9,10,13,19,\{47\}, 101,117,134,139,181$

$1,161,3,4,5,9,10,13,19,\{47\}, 117,134,139,181$

$1,17 \quad 1,3,4,5,9,10,13,19,40,\{47\}, 117,134,181, * 219$

77 Die Folie war während eines 2008 unter Leitung von S. G. Richter und Mitarbeit von F.-J. Schmitz, S. Moawad und M. H. O. Schulz durchgeführten Surveys zur Katalogisierung der Handschriften des Koptischen Museums zu Kairo nicht auffindbar. 


\begin{tabular}{|c|c|}
\hline 1,18 & $1,3,4,5,9,10,13,19,40,\{47\}, 103,117,134,139, * 219$ \\
\hline 1,19 & $1,3,4,5,9,10,13,19,40,\{47\}, 103,117,134,139,181, * 219$ \\
\hline 1,20 & $1,3,4,5,9,10,13,19,40,\{47\}, 117,134,139,181, * 219$ \\
\hline 1,21 & $1,3,4,5,9,10,13,19,40,\{47\}, 117,134,139,181, * 219$ \\
\hline 1,22 & $1,3,4,5,9,10,12,13,19,40,\{47\}, 117,134,139,181, * 219$ \\
\hline 1,23 & $1,3,4,5,9,10,12,13,19,40,\{47\}, 103,116,117,134,139,181, * 219$ \\
\hline 1,24 & $1,3,4,5,9,10,13,19,40,\{47\}, 103,116,126,134,139,181, * 219$ \\
\hline 1,25 & $1,3,4,5,9,10,13,19, * 40,\{47\}, 103,110,115,116,126,134,139,181, * 219$ \\
\hline 1,26 & $1,3,4,5,9,10,12,13,19,\{47\}, 110,115,116,126,134,181, * 219$ \\
\hline 1,27 & $1,3,4,5,9,10,12,13,19,\{47\}, 110,115,116,134,219$ \\
\hline 1,28 & $1,3,4,5,9,10,13,19,\{47\}, 110,115,116,139, * 219$ \\
\hline 1,29 & $1,3,4,5,9,10,13,19,\{47\}, 110,115,116,126,139,181, * 219$ \\
\hline 1,30 & $1,3,4,5,9,10,13,19,\{47\}, 110,115,116,126,139,181,219$ \\
\hline 1,31 & $1,3,4,5,9,10,13,19,40,\{47\}, 110,115,116,126,181,219$ \\
\hline 1,32 & $1,3,4,5,9,10,13,19,40,\{47\}, 103,110,115,116,126,181,219$ \\
\hline 1,33 & $1,3,4,5,9,10,12,13,19,40,\{47\}, 103,110,115,116,126,139,181, * 219$ \\
\hline 1,34 & $1,3,4,5,9,10, * 12,13,19,40,\{47\}, 103,110,115,116,126,139$ \\
\hline 1,35 & $1,3,4,5,9,10, * 12,13,14^{\mathrm{L}}, 19,40,\{47\}, 110,115,116,126$ \\
\hline 1,36 & $1,3,4,5,9, * 10,12,13,14^{\mathrm{L}}, 19,40,\{47\}, 110,115,116,126$ \\
\hline 1,37 & $1,3,4,5,9,10,13,14^{\mathrm{L}}, 19,40,\{47\}, 110,115,116,181$ \\
\hline 1,38 & $1,3,4,5,9,10,13,14^{\mathrm{L}}, 19,40,\{47\}, 103,110,115,116,126,139,181$ \\
\hline \multirow[t]{4}{*}{1,39} & $1,3,4,5,9,10,12,13,14^{\mathrm{L}}, 19,40,\{47\}, 103,110,115,116,126,139,181$ \\
\hline & Version $1: 3,4,5,9,12,13,19,40,110,116,126,181$ \\
\hline & Version 2: 1, 10, 14 $4^{\mathrm{L}}, 103,115,139$ \\
\hline & keine Zuordnung möglich: $\{47\}$ (Text unleserlich) \\
\hline 1,40 & $1,3,4,5,9,10,12,13,14^{\mathrm{L}}, 19,40,\{47\}, 103,110,115,116,126,139,181$ \\
\hline 1,41 & $1,3,4,5,9,10,13,14^{\mathrm{L}}, 19,40,\{47\}, 110,115,116,181,215$ \\
\hline 1,42 & $1,3,4,5,9,10,12,13,14^{\mathrm{L}}, 19, * 40,\{47\}, 110,115,116,126,181,215$ \\
\hline 1,43 & $1,3,4,5,9,10, * 12,13,14^{\mathrm{L}}, 15^{\mathrm{L}}, 19,\{47\}, 110,115,116,126,136,215,396^{\mathrm{L}}$ \\
\hline 1,44 & $1,3,4,5,9,10, * 12,13,14^{\mathrm{L}}, 15^{\mathrm{L}}, 19,\{47\}, 110,115,116,126,136,181$ \\
\hline 1,45 & $1,3,4,5,9,10,12,13,14^{\mathrm{L}}, 15^{\mathrm{L}}, 19,40,\{47\}, 110,115,116,126,136,181,297^{\mathrm{L}}$ \\
\hline 1,46 & $1,3,4,5,9,10,12,13,14^{\mathrm{L}}, 15^{\mathrm{L}}, 19,40,\{47\}, 110,115,116,126,136,181,297^{\mathrm{L}}$ \\
\hline 1,47 & $1,3,4,5,9,10, * 12,13,14^{\mathrm{L}}, 15^{\mathrm{L}}, 19,40,\{47\}, 110,115,116,126,136,167,181$ \\
\hline 1,48 & $1,3,4,5,9,10,12,13,14^{\mathrm{L}}, 15^{\mathrm{L}}, 19,40,\{47\}, 110,115,116,126,136,167,181$ \\
\hline 1,49 & $1,3,4,5,9,10,12,13,14^{\mathrm{L}}, 15^{\mathrm{L}}, 19,40,\{47\}, 110,115,116,126,136,167,181$ \\
\hline 1,50 & $1,3,4,5,9,10, * 12,13,14^{\mathrm{L}}, 15^{\mathrm{L}}, 19,40,\{47\}, 110,115,116,126,136,167, * 181,215$ \\
\hline 1,51 & $1,3,4,5,9,10,12,13,14^{\mathrm{L}}, 15^{\mathrm{L}}, 19,40,\{47\}, 110,115,116,126,136,167,181,215$ \\
\hline 2,1 & $1,3,4,5,9,10,12,13,14^{\mathrm{L}}, 15^{\mathrm{L}}, 19,\{47\}, 110,115,116,126,136,167,181$ \\
\hline 2,2 & $1,3,4,5,9,10,13,14^{\mathrm{L}}, 15^{\mathrm{L}}, 19,\{47\}, 110,115,116,126,136,167,181$ \\
\hline 2,3 & $1,3,4,5,9,10,13,14^{\mathrm{L}}, 15^{\mathrm{L}}, 19,\{47\}, 110,115,116,126,136,167,181,196$ \\
\hline 2,4 & $1,3,4,5,9,13,14^{\mathrm{L}}, 15^{\mathrm{L}}, 19,\{47\}, 110,115,116,126,136,167,181,196$ \\
\hline 2,5 & $1,3,4,5,9,10,12,13,14^{\mathrm{L}}, 15^{\mathrm{L}}, 19,\{47\}, 110,115,116,126,136,167,181,196, * 326^{\mathrm{L}}$ \\
\hline 2,6 & $\begin{array}{l}1,3,4,5,9,10,12,13,14^{\mathrm{L}}, 15^{\mathrm{L}}, 19,\{47\}, 110,115,116,126,136,167,181,196, * 251,294^{\mathrm{L}}, \\
326^{\mathrm{L}}\end{array}$ \\
\hline 2,7 & $1,3,4,5,9,10,13,14^{\mathrm{L}}, 15^{\mathrm{L}}, 19,\{47\}, 110,115,116,126,136,167,181,196,251,294^{\mathrm{L}}, 326^{\mathrm{L}}$ \\
\hline 2,8 & $1,3,4,5,9,10,13,14^{\mathrm{L}}, 15^{\mathrm{L}}, 19,\{47\}, 110,115,116,126,136,167,181,196,251,294^{\mathrm{L}}, 326^{\mathrm{L}}$ \\
\hline
\end{tabular}


$1,3,4,5,9,10,12,13,14^{\mathrm{L}}, 15^{\mathrm{L}}, 19,\{47\}, 110,115,116,126,136,167,196,251,294^{\mathrm{L}}, 326^{\mathrm{L}}$ $1,3,4,5,9,10,12,13,14^{\mathrm{L}}, 15^{\mathrm{L}}, 19,\{47\}, 110,115,116,126,136,167,181,196,251,294^{\mathrm{L}}$, $326^{\mathrm{L}}$

$$
1,3,4,5,9,10,14^{\mathrm{L}}, 15^{\mathrm{L}}, 19,\{47\}, 110,115,116,126,136,167,181,196,251,294^{\mathrm{L}}, 326^{\mathrm{L}}
$$
$1,3,4,5,9,10,12,19,\{47\}, 110,115,116,126,136,167,174,181,196,295^{\mathrm{L}}$, CGC 8086 $1,3,4,5,9,10,19,\{47\}, 106,110,115,116,126,136,167,174,181,196,295^{L}$, CGC 8086 $1,3,4,5,9,10,19,\{47\}, 106,110,115,116,126,136,167,174,181,190,196,295^{\mathrm{L}}$ $1,3,4,5,9,10,12,19,\{47\}, 106,110,115,116,126,136,167,174,181,190,196,295^{\mathrm{L}}$, CGC 8086

\section{$1,3,4,5,9,10,12,19,\{47\}, 106,110,115,116,126,136,167,174,196,295^{\mathrm{L}}$}

$1,3,4,5,9,10,19,\{47\}, 106,110,115,116,126,136,167,174,196,295^{L}$, CGC 8086

$1,3,4,5,9,10,12,19,\{47\}, 80,106,110,115,116,126,136,167,174,196,294^{\mathrm{L}}, 25^{\mathrm{L}}$, CGC 8086

$1,3,4,5,9,10,12,19,\{47\}, 80,106,110,115,116,126,136,167,174,181,196,294^{\mathrm{L}}$, $295^{\mathrm{L}}$, *CGC 8086

\section{$1,3,4,5,9,10, * 13,19,\{47\}, 80,106,110,115,116,126,136,167,174,181,196,294^{\mathrm{L}}$,} $295^{\mathrm{L}}$, CGC 8086

$1,3,4,5,9,10,13,19,\{47\}, 80,106,115,116,126,136,167,181,196,294^{\mathrm{L}}, 295^{\mathrm{L}}, \mathrm{CGC}$ 8086

$1,3,4,5,9,10,12,13,19,\{47\}, 80,106,110,115,116,126,136,167,181,196,294^{\mathrm{L}}, 295^{\mathrm{L}}$, CGC 8086

$1,3,4,5,9,10,12,19,\{47\}, 80,106,110,115,116,126,136,167,174,181,196,294^{\mathrm{L}}$ $1,3,4,5,9,10,19,80,106,115,116,126,136,167,174,177,181,196,294^{\mathrm{L}}$ $1,3,4,5,9,10,13,19,80, * 88,106,110,115,116,126,136,167,174,177,196,294^{\mathrm{L}}$

$1,3,4,5,9,10,12,13,14^{\mathrm{L}}, 15^{\mathrm{L}}, 19,80, * 88,106,115,116,126,136,167,174,177,196$, $306^{\mathrm{L}}$

$1,3,4,5,9,10,12,13,14^{\mathrm{L}}, 15^{\mathrm{L}}, 19,80, * 82,88,106,115,116,117,126,136,167,174,177$, $178,181,196,306^{\mathrm{L}}$

$1,4,5,9,10,13,14^{\mathrm{L}}, 15^{\mathrm{L}}, 19,80, * 82,106,115,116,117,126,136,167,174,177,178,181$, $196,306^{\mathrm{L}}$

$1,4,5,9,10,12,13,14^{\mathrm{L}}, 15^{\mathrm{L}}, 19,80,106,115,116,117,126,136,137,167,174,177,178$, $181,196,306^{\mathrm{L}}$

$1,4,5,9,10,13,14^{\mathrm{L}}, 15^{\mathrm{L}}, 19,106,115,116,117,136,137,167,174,177,178,181,196$, $306^{\mathrm{L}}$

$1,4,5,9,10,12,13,14^{\mathrm{L}}, 15^{\mathrm{L}}, 19,106,115,116,117,136,137,167,174,177, * 178,181,196$, $306^{\mathrm{L}}$

$1,4,5,9,10,12,14^{\mathrm{L}}, 15^{\mathrm{L}}, 19,106,115,116,117,136,137,167,177, * 178,196,306^{\mathrm{L}}$

$1,4,5,9,10,13,14^{\mathrm{L}}, 15^{\mathrm{L}}, 19,106,115,116,117,136,137,167,177,178,181,196,306^{\mathrm{L}}$

$1,4,5,9,10,14^{\mathrm{L}}, 15^{\mathrm{L}}, 19, * 82,106,115,116,117,136,137,167,177,178,181,196$

$1,3,4,5,9,10,12,14^{\mathrm{L}}, 15^{\mathrm{L}}, 19,82,106,115,116,117,136,137,{ }^{*} 149,167,177,178,181$, 196

$1,3,4,5,9,10,12,14^{\mathrm{L}}, 15^{\mathrm{L}}, 19,106,115,116,136,137, * 149,167,177,181,196$

$1,3,4,5,9,10,15^{\mathrm{L}}, 19,106,115,116,136,137,167,177,178,181,196$ $1,3,4,5,9,10,12,15^{\mathrm{L}}, 19,106,115,116,136,137,167,177,178,181,196$ $1,3,4,5,9,10,12,15^{\mathrm{L}}, 19,106,115,116,136,137,167,177,178,181,190,196$ $1,3,4,5,9,10,15^{\mathrm{L}}, 19,106,115,116,136,137, * 149,167,177,178,181,190$ 
$3,16 \quad 3,4,5,9,10,12,19,106,115,116,136,137, * 149,167,178,190$

$3,17 \quad 1,3,4,5,9,10,12,19, * 88,106,115,116,136,137,141, * 149,167,190,402$

$3,181,3,4,5,9,10,19, * 88,105,106,115,116,136,141,167,181,190,402$

$3,191,3,4,5,9,10,12,19, * 88,105,115,116,136,141,167,181,402$

$3,201,3,4,5,9,10,12,19,105,115,116,136,141,149,167,181,402$

$3,21 \quad 1,3,4,5,9,10,19,115,116,136,141,149,167,181,402$

$3,22 \quad 1,3,4,5,9,10,19,112,115,116,136,141, * 149,167,181$

$3,23 \quad 1,3,4,5,9,10,12,19,112,115,116,136,139,141,167,181$

$3,24 \quad 1,3,4,5,9,10,12,19,112,115,116,136,139,141,167$

$3,25 \quad 1,3,4,5,9,10,19,112,115,116,136,139,141,167$

$3,261,3,4,5,9,10,12,13,19,112,115,116,136,139,141,149,167,296^{\mathrm{L}}$

3,27

3,28

3,29

3,30

3,31

$1,3,4,5,9,10,12,13,19,110,115,116, * 126,136,139,141, * 149,167,181,296^{\mathrm{L}}$

$1,3,4,5,9,10,13,19,110,115,116,126,136,139,141,167,181,296^{\mathrm{L}}$

$1,3,4,5,9,10,12,13,19,110,112,115,116,136,139,141,167,181,187$

$1,3,4,5,9,10,13,19,110,112,115,116,136,139,141,167,181,187$

Version 1: 3, 4, 5, 9, 13, 19, 110, 112, 116, 167, 181, 187

Version 2: 1, 10, 115, 136, 139, 141

3,32

1 , 3, 4, 5, 9, 10, 13, 19, 110, 112, 115, 116, 136, 139, 141, 167, 181, 187

3,33

$1,3,4,5, * 8,9,10,12,13,19,110,112,115,116,136,139,141,167,187,195$

3,34

$1,3,4,5,8,9,10,13,19,110,115,116,136,139,141,167,187,195$

3,35

$1,3,4,5,8,9,10,13,19,110,115,116,136,139,141,167,181,187$

3,36

$1,3,4,5,8,9,10,13,19,110,115,116,136,139,141,167,181,187$

4,1

4,2

4,3

4,4

4,5

4,6

4,7

$1,3,4,5,8,9,10,12,13,19,110,112,115,116,136,139,141,167,181,187,454$

$1,3,4,5,8,9,10,12,13,19,110,112,115,116,136,139,141,167,181,187,193, * 454$

$1,3,4,5,9,10,13,19,110,115,116,136,139,141,167,181,187,193$

$1,3,4,5,9,10,13,19,110,115,116,136,139,141,167,181,187,193$

$1,3,4,5,9,10,13,19,115,116,136,139,141,167,181,187,193$

$1,3,4,5,9,10,12,13,19,115,116,136,139,141,167,181,187,193,291^{\mathrm{L}}, 301^{\mathrm{L}}, 339^{\mathrm{L}}$

$1,3,4,5,9,10,12,13,19,112,115,116,136,139,142,167,187,193,291^{\mathrm{L}}, 301^{\mathrm{L}}, 310^{\mathrm{L}}, 339^{\mathrm{L}}$

$1,3,4,5,9,10,13,19,112,115,116,136,139,142,167,187,190,193,291^{\mathrm{L}}, 301^{\mathrm{L}}, 310^{\mathrm{L}}$, $339^{\mathrm{L}}$

$4,8 \quad 1,3,4,5,9,10,13,19,112,115,116,136,139,142,167,187,190,193,291^{\mathrm{L}}, 301^{\mathrm{L}}, 310^{\mathrm{L}}$, $339^{\mathrm{L}}$

$4,9 \quad 1,3,4,5,9,10,12,19,112,115,116,124,126,136,139,142,167,181,187,190,193,195$, $291^{\mathrm{L}}, 301^{\mathrm{L}}, 310^{\mathrm{L}}, 339^{\mathrm{L}}$

$4,101,3,4,5,9,10,12,19,115,116,124,126,142,167,181,193,291^{\mathrm{L}}, 301^{\mathrm{L}}, 310^{\mathrm{L}}, 339^{\mathrm{L}}$

$4,11 \quad 1,3,4,5,9,10,12,19,115,116,124,142,167,181,291^{\mathrm{L}}, 301^{\mathrm{L}}, 310^{\mathrm{L}}, 339^{\mathrm{L}}$

$4,121,3,4,5,9,10,12,19,112,115,116,124,142,167,181,291^{\mathrm{L}}, 310^{\mathrm{L}}, 339^{\mathrm{L}}$

$4,13 \quad 1,3,4,5,9,10,19,112,115,116,124,142,167,181,291^{\mathrm{L}}, 301^{\mathrm{L}}, 310^{\mathrm{L}}$

$4,141,3,4,5,9,10,12,19,112,115,116,124,142,167,190,291^{\mathrm{L}}, 301^{\mathrm{L}}, 310^{\mathrm{L}}$

$4,151,3,4,5,9,10,12,13,19,112,115,116,124,142,167,190,291^{\mathrm{L}}, 301^{\mathrm{L}}$

$4,161,3,4,5,9,10,13,19,112,115,116,124,142,167,190,291^{\mathrm{L}}$

$4,17 \quad 1,3,4,5,9,10,13,19,102,112,115,116,124,142,167,190,291^{\mathrm{L}}$

4,18 1, 3 (Text ursprünglich teils ausgelassen und nachgetragen. Es lässt sich nicht genau verzeichnen, was ursprünglich und was in unregelmäßiger Schrifttype [zweite Hand?] nachgetragen ist.), 4, 5, 9, 10, 13, 19, 102, 112, 115, 116, 124, 142, 167, 181, 190, $291^{\mathrm{L}}$ 


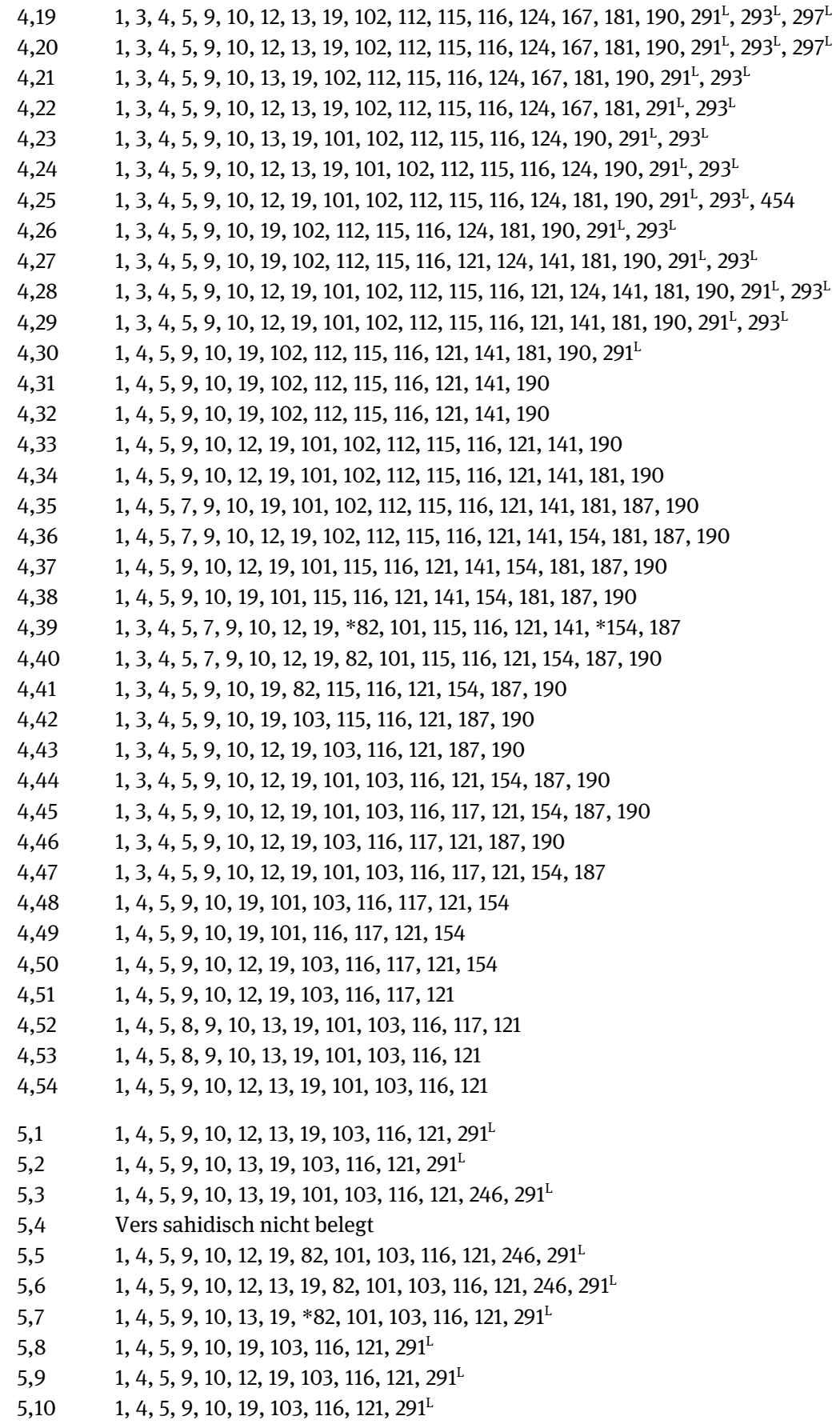




\begin{tabular}{|c|c|}
\hline 5,11 & $1,4,5,9,10,19,103,116,121,291^{\mathrm{L}}$ \\
\hline 5,12 & $1,4,5,9,10,19,116,121,291^{\mathrm{L}}$ \\
\hline 5,13 & $1,4,5,9,10,12,19,105,116,121,246,291^{\mathrm{L}}$ \\
\hline 5,14 & $1,4,5,9,10,19,105,116,121,136,246,291^{\mathrm{L}}$ \\
\hline 5,15 & $1,4,5,9,10,19,105,116,121,136$ \\
\hline 5,16 & $1,4,5,9,10,12,19,105,116,121,136$ \\
\hline 5,17 & $1,4,5,9,10,12,13,19,105,116,121,136$ \\
\hline 5,18 & $1,4,5,9,10,12,13,19,101,105,116,121,136$ \\
\hline 5,19 & $1,4,5,9,10,12,13,19,56,101,105,116,121,136,246$ \\
\hline 5,20 & $1,4,5,9,10,13,19,101,105,116,121,136,246$ \\
\hline 5,21 & $1,4,5,9,10,13,15^{L}, 19,101,105,116,121,136$ \\
\hline 5,22 & $1,4,5,9,10,12,13,15^{\mathrm{L}}, 19,101,105,116,121,136$ \\
\hline 5,23 & $1,4,5,9,10,13,15^{\mathrm{L}}, 19,56,116,121,136$ \\
\hline 5,24 & $1,4,5,9,10,12,15^{L}, 19,105,116,121,136,167$ \\
\hline 5,25 & $1,4,5,9,10,12,15^{\mathrm{L}}, 19,56,105,116,121,136,167,246$ \\
\hline 5,26 & $1,4,5,9,10,15^{L}, 19,116,121,136,167,246$ \\
\hline 5,27 & $1,4,5,9,10,12,15^{\mathrm{L}}, 19,116,121,136,167$ \\
\hline 5,28 & $1,4,5,9,10,12,15^{\mathrm{L}}, 19,116,121,136,167$ \\
\hline 5,29 & $1,4,5,9,10,15^{\mathrm{L}}, 19,56,116,121,136,167$ \\
\hline 5,30 & $1,4,5,9,10,12,13,19,116,121,136,167$ \\
\hline 5,31 & $1,4,5,9,10,13,19,116,121,167$ \\
\hline 5,32 & $1,4,5,9,10,13,19,82,101,116,121,167$ \\
\hline 5,33 & $1,4,5,9,10,13,19,82,101,116,121,167,396^{\mathrm{L}}$ \\
\hline 5,34 & $1,4,5,9,10,12,13,19,101,116,121,167,194$ \\
\hline 5,35 & $1,4,5,9,10,12,13,19,116,121,167,194$ \\
\hline 5,36 & $1,3,4,5,9,10,12,13,19,116,121,167$ \\
\hline 5,37 & $1,3,4,5,9,10,12,13,19,116,121,167$ \\
\hline 5,38 & $1,3,4,5,9,10,13,19,116,121,167$ \\
\hline 5,39 & $1,3,4,5,9,10,12,13,19,116,121,167,194$ \\
\hline 5,40 & $1,3,4,5,9,10,13,19,116,121,167,194$ \\
\hline 5,41 & $1,3,4,5,9,10,13,19,116,121,167$ \\
\hline 5,42 & $1,3,4,5,9,10,13,19,116,121,139,167$ \\
\hline 5,43 & $1,3,4,5,9,10,13,19,116,121,139,167$ \\
\hline 5,44 & $1,3,4,5,9,10,13,19,116,121,139,167$ \\
\hline 5,45 & $1,3,4,5,9,10,13,19,116,121,139,167,194$ \\
\hline 5,46 & $1,3,4,5,9,10,13,19,116,121,167,194$ \\
\hline 5,47 & $1,3,4,5,9,10,13,19,116,121,139,167,194$ \\
\hline 6,1 & $1,3,4,5,9,10,19,40,60,82,116,121,139,167,347^{\mathrm{L}}$ \\
\hline 6,2 & $1,3,4,5,9,10,19,60,82,116,121,139,167,347^{\mathrm{L}}$ \\
\hline 6,3 & $1,3,4,5,9,10,19,116,121,139,167,347^{\mathrm{L}}$ \\
\hline 6,4 & $1,3,4,5,9,10,19,82,116,121,139,167, * 347^{\mathrm{L}}$ \\
\hline 6,5 & $1,3,4,5,9,10,15^{\mathrm{L}}, 19,82,116,121,139,167,194,347^{\mathrm{L}}$ \\
\hline 6,6 & $1,3,4,5,9,10,15^{\mathrm{L}}, 19,116,121,139,194,347^{\mathrm{L}}$ \\
\hline 6,7 & $1,3,4,5,9,10,15^{\mathrm{L}}, 19,82,116,121,139,194,347^{\mathrm{L}}$ \\
\hline 6,8 & $1,3,4,5,9,10,15^{\mathrm{L}}, 19,82,84,116,121,139,294^{\mathrm{L}}, 347^{\mathrm{L}}$ \\
\hline 6,9 & $1,3,4,5,9,10,15^{\mathrm{L}}, 19,84,116,121,139,294^{\mathrm{L}}, 347^{\mathrm{L}}$ \\
\hline
\end{tabular}




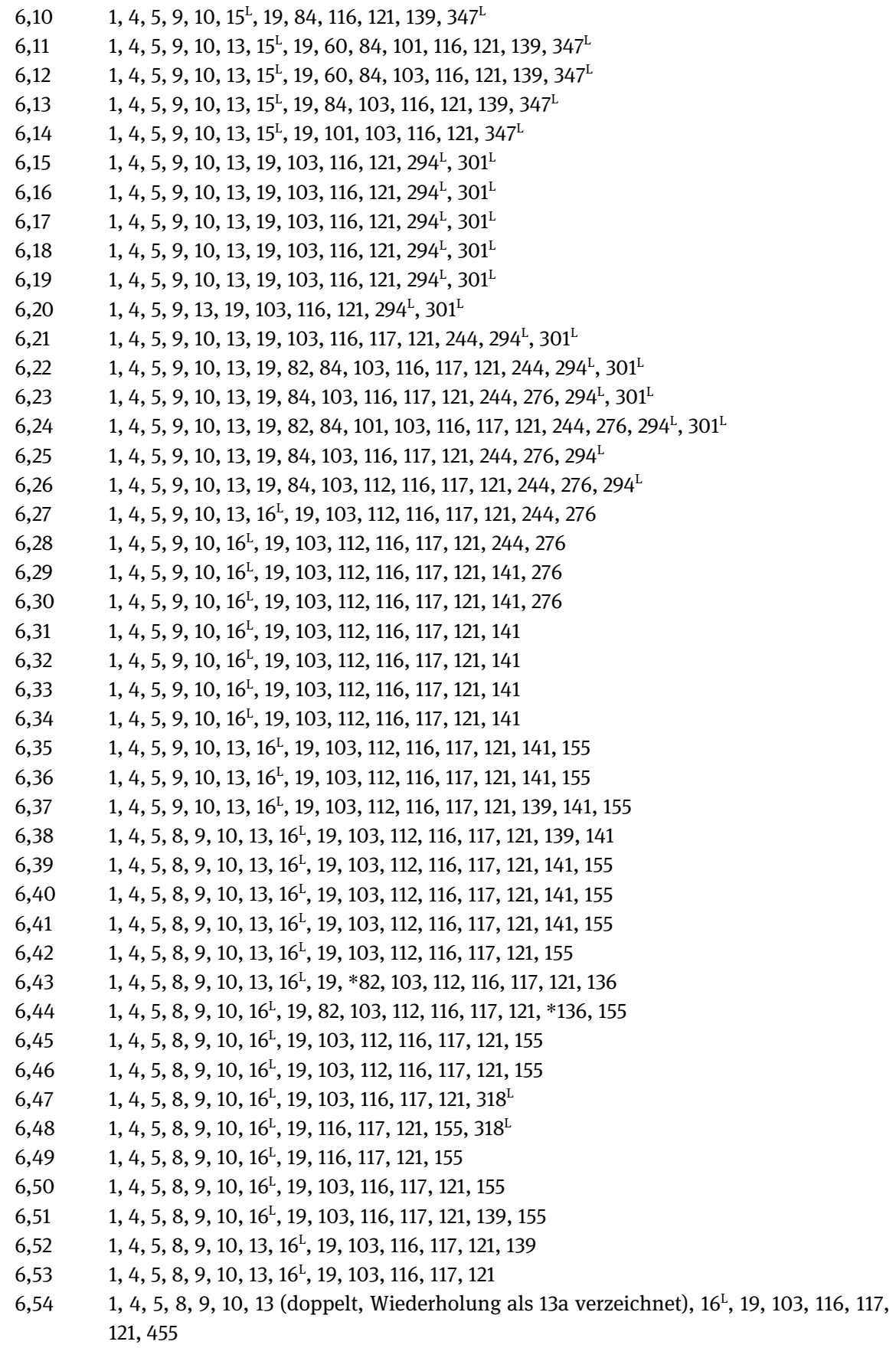


$6,551,4,5,8,9,10,13$ (doppelt, Wiederholung als 13a verzeichnet), 16 ${ }^{\mathrm{L}}, 19,103,116,117$, 121,455

6,56 1, 4, 5, 8, 9, 10, 13 (doppelt, Wiederholung als 13a verzeichnet), 16 ${ }^{\mathrm{L}}, 19,103,116,117$, 121

$6,57 \quad 1,4,5,8,9,10,13,16^{\mathrm{L}}, 19,103,116,117,121$

$6,581,4,5,8,9,10,13,16^{\mathrm{L}}, 19,103,116,117,121,136$

$6,591,4,5,9,10, * 13,19,103,116,121$

$6,601,4,5,9,10,19,103,116,121$

$6,61 \quad 1,4,5,9,10,19,103,116,121, * 136,162,455$

$6,621,4,5,9,10,19,103,116,121, * 136,162,293^{\mathrm{L}}, * 455$

$6,63 \quad 1,4,5,9,10,19,103,105,116,121,136,162,293^{\mathrm{L}}$

$6,64 \quad 1,4,5,8,9,10,19,103,105,116,121,136,162,293^{\mathrm{L}}$

$6,651,4,5,8,9,10,19,103,105,116,121,136,139,162,293^{\mathrm{L}}$

$6,661,4,5,8,9,10,19,103,105,116,121, * 136,139,162,293^{\mathrm{L}}$

$6,67 \quad 1,4,5,8,9,10,19,103,105,116,121,136,139,162,293^{\mathrm{L}}$

$6,681,4,5,8,9,10,13,19,103,105,116,117,121,136,139,162,293^{\mathrm{L}}$

$6,691,4,5,8,9,10,13,19,103,105,116,117,121,136,139,162,293^{\mathrm{L}}$

$6,70 \quad 1,4,5,7,9,10,13,19,103,105,116,117,121,136,139,162$

$6,71 \quad 1,4,5,7,9,10,13,19,103,105,116,117,121,123,136,139,162$

$7,1 \quad 1,4,5,7,9,10,13,19,103,105,116,117,121,123,136,139,162$

$7,2 \quad 1,4,5,9,10,13,19,103,116,117,121,123,136,139$

$7,3 \quad 1,4,5,7,9,10,13,19,103,116,117,121,123,136,139,162$

$7,4 \quad 1,4,5,7,9,10,13,19,103,116,117,121,123,136,139,141,162$

$7,5 \quad 1,4,5,7,9,10,13,19,103,116,117,121,123,136,139,141,162$

$7,61,4,5,9,10,13,19,82,101,103,116,117,121,136,139,141,162$

$7,7 \quad 1,4,5,9,10,13,19,82,101,103,116,117,121,136,139,141,162,350$

$7,8 \quad 1,4,5,9,10,13,19,101,103,116,117,121,136,139,141,162$

$7,9 \quad 1,4,5,9,10,13,19,101,103,116,117,121,136,139,141,162$

$7,101,4,5,9,10,13,19,101,103,116,117,121,123,136,139,141,162$

$7,11 \quad 1,4,5,9,10,13,19,103,116,117,121,123,141,162$

$7,12 \quad 1,4,5,9,10,13,19,82,101,103,116,117,121,123,141,162$

$7,13 \quad 1,4,5,9,10,13,19, * 82,103,116,117,121,123,141,162,285$

$7,14 \quad 1,4,5,9,10,13,15^{\mathrm{L}}, 19,103,116,117,121,123,141,162,285,291^{\mathrm{L}}$

$7,15 \quad 1,4,5,9,10,13,15^{\mathrm{L}}, 19,103,116,117,121,123,141,162,285,291^{\mathrm{L}}$

$7,161,4,5,9,10,15^{\mathrm{L}}, 19,103,116,117,121,123,141,162,285,291^{\mathrm{L}}$

$7,17 \quad 1,4,5,9,10,15^{\mathrm{L}}, 19,101,103,116,117,121,141,162,285,291^{\mathrm{L}}$

$7,18 \quad 1,4,5,9,10,15^{\mathrm{L}}, 19,103,116,117,121,132,141,162,234,285$

$7,191,4,5,9,10,15^{\mathrm{L}}, 19,116,117,121,132,162,234,285$

$7,20 \quad 1,4,5,9,10,15^{\mathrm{L}}, 19,101,116,117,121,132,162,234$

$7,21 \quad 1,4,5,9,10,15^{\mathrm{L}}, 19,101,116,117,121,123,132,162,234$

$7,22 \quad 1,4,5,9,10,15^{\mathrm{L}}, 19,82,101,116,117,121,123,132,162,234$

$7,23 \quad 1,4,5,9,10,15^{\mathrm{L}}, 19,82,101,112,116,117,123,132,162,234$

$7,24 \quad 1,4,5,9,10,15^{\mathrm{L}}, 19,82,101,112,116,117,123,132, * 162,234$

$7,25 \quad 1,4,5,9,10,19,101,112,115,116,117,123,132,162,234$

$7,26 \quad 1,4,5,9,10,19,101,112,115,116,117,121,123,132,162,234$

$7,27 \quad 1,4,5,9,10,19,112,115,116,117,121,132,162,234,270$ 


\section{7,41}

7,42

7,43

7,44

$1,4,5,9,10,19,82,101,112,115,116,117,121,132,136,162,234,270$

$1,4,5,9,10,19,112,115,116,117,121,125,134,136,162,234,250,270$

$1,4,5,9,10,19,112,115,116,117,121,123,125,134,136,162,234,250,270$

$1,4,5,9,10,19,112,115,116,117,121,123,125,134,136,162,234,250,270$

$1,4,5,9,10,19, * 82,101,112,115,116,117,121,123,125,134,136,162,234,250,270$

$1,4,5,9,10,19,82,101,112,115,116,117,121,123,125,134,136,159,162,234,250$

$1,4,5,9,10,19,112,115,116,117,123,125,136,159,162,234,250$

$1,4,5,9,10,19,101,112,115,116,117,123,125,136,159,162,197,234,250$

$1,4,5,9,10,19,82,101,112,115,116,117,125,136,162,197,234,250$

$1,4,5,9,10,19,82,101,112,115,116,117,125,136,159,162,197,234,250,302^{\mathrm{L}}, 305^{\mathrm{L}}$ $1,4,5,9,10,19,82,112,115,116,117,125,136,159,162,197,234,250,302^{\mathrm{L}}, 305^{\mathrm{L}}, 343^{\mathrm{L}}$ $1,4,5,9,10,19,112,115,116,117,125,134,136,162,197,234,250,302^{\mathrm{L}}, 305^{\mathrm{L}}, 343^{\mathrm{L}}$ $1,4,5,9,10,19,112,115,116,117,125,134,136, * 159,162,197,234,250,302^{\mathrm{L}}, 305^{\mathrm{L}}$, $343^{\mathrm{L}}$

$1,4,5,9,10,19,103,112,115,116,117,125,134,136,159,197,250,302^{\mathrm{L}}, 305^{\mathrm{L}}$

$1,4,5,9,10,19,103,105,112,115,116,117,125,134,136,159,197,250,302^{\mathrm{L}}, 305^{\mathrm{L}}, 343^{\mathrm{L}}$ $1,4,5,9,10,19,103,105,112,115,116,117,125,134,136,159,197,250,302^{\mathrm{L}}, 305^{\mathrm{L}}, 343^{\mathrm{L}}$ $1,4,5,9,10,19,103,105,112,115,116,117,125,136,139,159,197,250,302^{\mathrm{L}}, 305^{\mathrm{L}}, 343^{\mathrm{L}}$ $1,4,5,9,10,19,103,105,112,115,116,117,125,136,139,159,197,250,302^{\mathrm{L}}, 305^{\mathrm{L}}$ $1,4,5,9,10,19,103,105,112,116,117,125,136,139,159,197,250,305^{\mathrm{L}}$ $1,4,5,9,10,19,82,103,105,112,116,117,125,136,139,159,197,250$ $1,4,5,9,10,19,82,103,105,112,116,117,125,134,136,139,159,197,250$ $1,4,5,9,10,13,19,82,103,105,112,116,117,125,134,136,139,159,197,250$ $1,4,5,9,10,13,19,82,103,105,112,116,117,125,134,136,139,159,197,210$ $1,4,5,9,10,13,19,103,105,112,116,117,125,134,136,139,159,197,210$

7,53-8,11 Die Verse sind in sahidischen Handschriften nicht belegt (pericope adulterae).

$\begin{array}{ll}8,12 & 1,4,5,9,10,13,16^{\mathrm{L}}, 19,82,103,105,112,116,117,125,134,136,139,159,197,210 \\ 8,13 & 1,4,5,9,10,13,16^{\mathrm{L}}, 19,82,103,105,112,116,117,125,134,136,139,159,197, * 210 \\ 8,14 & 1,4,5,9,10,13,16^{\mathrm{L}}, 19,82,103,112,116,117,125,134,136,139,159,197, * 210 \\ 8,15 & 1,4,5,9,10,13,16^{\mathrm{L}}, 19,103,112,116,117,125,136,139,159,197, * 210 \\ 8,16 & 1,4,5,9,10,13,16^{\mathrm{L}}, 19,103,112,116,117,125,126,136,139,159,197,210 \\ 8,17 & 1,4,5,9,10,13,16^{\mathrm{L}}, 19,103,112,116,117,125,126,136,139,159,197, * 210 \\ 8,18 & 1,4,5,9,10,13,16^{\mathrm{L}}, 19,103,112,116,117,125,126,136,139,159,197, * 210 \\ 8,19 & 1,4,5,9,10,13,16^{\mathrm{L}}, 19,103,112,116,117,125,126,136,139,159,197 \\ 8,20 & 1,4,5,9,10,13,16^{\mathrm{L}}, 19,103,112,116,117,125,126,136,139,159,197,285 \\ 8,21 & 1,4,5,9,10,13,16^{\mathrm{L}}, 19,103,112,116,117,125,126,136,159,197,216,285 \\ 8,22 & 1,4,5,9,10,13,16^{\mathrm{L}}, 19,103,105,112,116,117,125,126,136,159,197,216,285 \\ 8,23 & 1,4,5,9,10,13,16^{\mathrm{L}}, 19,86,103,105,112,116,117,125,126,136,155,159,197,216,285 \\ 8,24 & 1,4,5,9,10,16^{\mathrm{L}}, 19,105,116,125,126,136,155,159,197,216,285 \\ 8,25 & 1,4,5,9,10,13,16^{\mathrm{L}}, 19,105,115,116,125,126,136,155,159,197,216,285 \\ 8,26 & 1,4,5,9,10,13,16^{\mathrm{L}}, 19,105,115,116,125,126,136,155,159,216,285 \\ 8,27 & 1,4,5,9,10,13,16^{\mathrm{L}}, 19,105,115,116,125,126,136,155,159,216,285 \\ 8,28 & 1,4,5,9,10,13,16^{\mathrm{L}}, 19,105,115,116,125,126,136,155,159,285 \\ 8,29 & 1,4,5,9,10,13,19,105,115,116,125,126,136,155,159,285 \\ 8,30 & 1,4,5,9,10, * 13,19,105,115,116,125,126,136,155,159,285\end{array}$


$8,31 \quad 1,4,5,9,10,13,19,82,105,115,116,125,126,136,155,159,185,285$

$8,32 \quad 1,4,5,9,10,13,19,105,115,116,125,126,136,155,159,185,285$

$8,331,4,5,9,10,13,19,105,115,116,123,125,126,136,155,159,185,238,285$

$8,34 \quad 1,4,5,9,10,13,105,115,116,123,125,126,136,155,159,238$

$8,35 \quad 1,4,5,9,10,13,19,101,105,115,116,123,125,136,155,185,238$

$8,361,4,5,9,10,13,19,101,105,115,116,123,125,136, * 139,185,238$

$8,37 \quad 1,4,5,9,10,13,19,82,101,105,110,115,116,123,125,136,139,141,155,238$

$8,381,4,5,9,10,13,19,82,105,106,110,115,116,123,125,136,139,141,155,238$

$8,391,4,5,9,10,13,19,105,106,110,115,116,123,125,136,139,141,155,238$

$8,40 \quad 1,4,5,9,10,13,19,105,106,110,115,116,123,125,136,139,141,238,285$

$8,41 \quad 1,4,5,9,10,13,19,105,106,110,115,116,123,125,136,139,238,285$

$8,42 \quad 1,4,5,9,10,13,19,105,106,110,115,116,123,125,136,139,141,238,285$

$8,43 \quad 1,4,5,9,10,13,19,105,106,110,115,116,125,136,139,141,238,285$

$8,441,4,5,9,10,13,19,101,105,106,110,115, * 116,125,136,139,141,238,285$

$8,45 \quad 1,4,5,9,13,19,101,105,106,110,125,136,139,238,285$

$8,461,4,5,9,10,13,19,101,105,106,110,125,136,139,238,285$

$8,47 \quad 1,4,5,9,10,13,19,105,106,125,136,139,238,285$

$8,48 \quad 1,4,5,9,10,13,19,82,105,106,125,127,136,139,238,285$

$8,49 \quad 1,4,5,9,10,13,19,82,105,106,125,127,136,139,238,285$

$8,50 \quad 1,4,5,9,13,19,82,105,106,125,127,136,238,285$

$8,51 \quad 1,4,5,9,10,13,16^{\mathrm{L}}, 19,82,105,106,125,127,136,238,285$

$8,521,4,5,9,10,13,16^{\mathrm{L}}, 19,105,106,125,127,136,238,285$

$8,53 \quad 1,4,5,9,10,13,16^{\mathrm{L}}, 19,82,105,106,125,127,136,238,285$

$8,54 \quad 1,4,5,9,10,13,16^{\mathrm{L}}, 19,82,105,106,109,125,127,136,238,285$

$8,551,4,5,9,10,13,16^{\mathrm{L}}, 19,82,105,106,109,125,127,136,285$

$8,561,4,5,9,10,13,16^{\mathrm{L}}, 19,105,106,109,125,127,136,285$

$8,57 \quad 1,4,5,9,10,13,16^{\mathrm{L}}, 19,105,106,109,125,127,136,285$

$8,581,4,5,9,10,12,13,16^{\mathrm{L}}, 19,105,106,109,125,127,136,141,285$

$8,591,4,5,9,10,12,13,16^{\mathrm{L}}, 19,105,106,109,125,127,136,141,285$

$9,1 \quad 1,4,5,9,10,12,13,19,105,106,109,125,127,136,141,285$

$9,2 \quad 1,4,5,9,10,13,19,105,106,109,123,125,127,136,285$

$9,311,4,5,9,10,13,19,105,106,109,123,125,127,136,174,285$

$9,41,4,5,9,10,12,13,19,105,106,109,123,125,\{127\}, 136,174,285$

$9,5 \quad 1,4,5,9,10,12,19,105,106,109,123,125,\{127\}, 136,174,285$

$9,61,4,5,9,10,19,105,106,109,116,123,125,\{127\}, 136,141,174,285$

$9,711,4,5,9,10,13,19,103,105,106,109,115,116,123,125,\{127\}, 136,141,174,285$

$9,81,4,5,9,10,12,13,19,103,105,106,109,115,116,123,125,\{127\}, 136,174,285$

$9,91,4,5,9,10,19,103,105,106,109,115,116,123,125,\{127\}, 136,174,285$

$9,101,4,5,9,10,19,103,105,106,109,115,116,123,125,\{127\}, 136,174,285$

$9,11 \quad 1,4,5,9,10,12,13,19,103,105,106,109,115,116,123,125,\{127\}, 136,173,174,285$

$9,121,4,5,9,10,12,13,19,103,105,106,109,110,115,116,125,\{127\}, 136,173,174,285$

$9,131,4,5,9,10,13,19,82,103,105,106,109,110,115,116,125,\{127\}, 136,173,174,285$

$9,141,4,5,9,13,19,82,103,105,106,109,110,115,116,125,\{127\}, 136,173,174,285$

$9,151,4,5,9,10,19,82,102,103,105,106,109,110,115,116,125,\{127\}, 136,173,174,285$

$9,161,4,5,9,10,13,19,82,102,103,105,106,109,110,115,116,125,\{127\}, 136,173,174$, 285 
$9,171,4,5,9,10,12,19, * 82,102,103,105,106,109,110,115,116,125,127,136,173,174$, 285

$9,181,4,5,9,10,19,82,102,103,105,106,109,110,115,116,125,127,136,173,174,285$

$9,191,4,5,9,10,13,19,82,102,103,105,106,110,115,116,125,127,134,136,173,174,285$

$9,201,4,5,9,10,12,13,19,82,102,103,105,106,110,115,116,125,127,134,136,173,174$, 285, P.Bachit 255

9,21 $1,4,5,9,10,19,82,102,103,105,106,110,115,116,125,127,134,136,173,174,285$

9,22 $1,4,5,9,10,12,19,82,102,103,105,106,108,110,115,116,125,127,134,136,173,174$, 178, 218, 285, P.Bachit 255

$9,231,4,5,9,10,12,19,102,103,105,106,108,115,116,125,127,134,136,173,174,178$, 218, 285

9,24 1, 4, 5, 9, 10, 19, 102, 103, 105, 106, 108, 115, 116, 125, 127, 134, 136, 173, 174, 178, 218, 285

9,25 1, 4, 5, 9, *10, 12, 19, 102, 103, 105, 106, 108, 115, 116, 125, 127, 134, 136, 173, 174, 178, 218, 285

9,26 1, 4, 5, 9, 10, 19, 102, 103, 105, 106, 108, 115, 116, 125, 127, 134, 136, 173, 174, 218, 285

9,27 $1,4,5,9,10,19,102,103,105,106,108,115,125,127,134,136,173,174,218,285$

9,28

9,29

9,30 $1,4,5,9,10,12,19,82,102,103,105,106,108,125,127,134,136,142,174,178,218,285$ $1,4,5,9,10,12,19,82,102,103,108,125,127,134,136,142,174,218,285$ $1,4,5,9,10,19,82,102,103,108,125,127,134,136,142,174,218,285$

9,31

9,32

9,33

9,34

9,35

9,36

9,37

1, 4, 5, 9, 10, 19, 102, 103, 108, 125, 126, 127, 134, 136, 142, 174, 178, 218, 285

$1,4,5,9,10,19,102,103,108,125,126,127,134,136,174,178,218,285$

1, 4, 5, 9, 10, 12, 19, 102, 103, 108, 125, 126, 127, 134, 136, 174, 178, 218, 285

$1,4,5,9,10,19,102,103,108,125,126,127,134,136,142,174,178,218,285$

1, 4, 5, 9, 10, 19, 102, 103, 108, 125, 126, 127, 134, 136, 142, 174, 218, 285

1, 4, 5, 9, 10, 19, 102, 103, 108, 125, 126, 127, 134, 136, 142, 174, 218, 285

$1,4,5,9,10,19,102,103,108,125,126,127,134,136,142,174,218,285$

9,38 4, 5, 9, 10, 19, 102, 103, 108, 125, 126, 127, 134, 136, 142, 174, 218, 285

Version 1: 4, 5, 9, 10, 19, 125, 126, 174, 218

Version 2: 102, 103, 108, 127, 134, 136, 142, 285

9,39 1, 4, 5, 9, 10, 19, 102, 103, 108, 125, 126, 127, 134, 136, 174, 285

$9,401,4,5,9,10,19,102,103,108,125,126,127,134,136,141,174,285$

$9,411,4,5,9,10,19,102,103,125,126,127,134,136,141,174,285$

$10,1 \quad 1,4,5,9,10,19,102,103,126,127,134,136,141,174,285$

$10,2 \quad 1,4,5,9,10,19,102,103,106,126,127,134,136,141,285$

$10,3 \quad 1,4,5,9,10,19,102,103,106,126,127,134,136,141,285$

$10,4 \quad 1,4,5,9,10,19,102,103,106,126,127,134,136,141,285$

$10,5 \quad 1,4,5,9,10,19,102,103,106,126,127,134,136,141,285$

$10,61,4,5,9,10,19,82,102,103,106,126,127,134,136,141,285$

$10,7 \quad 1,4,5,9,10,15^{\mathrm{L}}$ (Versbeginn als Lesungseinleitung zur Perikope 10,11-18), 19, 82, 102, 103, 106, 126, 127, 134, 136, 141, 285

$10,8 \quad 1,4,5,9,10,19,82,103,106,115,126,127,134,136,141,285$

$10,91,4,5,9,10,14^{\mathrm{L}}, 19,103,106,115,126,127,134,136,141,285$

$10,101,4,5,9,10,14^{\mathrm{L}}, 19,103,106,115,126,127,134,136,141,285$

$10,111,4,5,9,10,14^{\mathrm{L}}, 15^{\mathrm{L}}, 19, * 101,103,106,115,127,136,141,285$

$10,121,4,5,9,10,14^{\mathrm{L}}, 15^{\mathrm{L}}, 19, * 101,103,106,115,127,136,285,339^{\mathrm{L}}, 407^{\mathrm{L}}$ 
$10,13 \quad 1,4,5,9,10,14^{\mathrm{L}}, 15^{\mathrm{L}}, 19, * 101,103,106,115,127,285,339^{\mathrm{L}}, 407^{\mathrm{L}}$

$10,141,4,5,9,10,14^{\mathrm{L}}\left(2 \times, 2\right.$. Mal als $14^{\mathrm{L}} \mathrm{a}$ verzeichnet $), 15^{\mathrm{L}}, 16^{\mathrm{L}}, 19,101,103,106,115,127$, $285,339^{\mathrm{L}}, 407^{\mathrm{L}}$

$10,151,4,5,9,10,14^{\mathrm{L}}\left(2 \times, 2\right.$. Mal als $14^{\mathrm{L}}$ a verzeichnet), $15^{\mathrm{L}}, 16^{\mathrm{L}}, 19,101,103,106,115,127$, $285,339^{\mathrm{L}}, 407^{\mathrm{L}}$

$10,161,4,5,9,10,12,14^{\mathrm{L}}\left(2 \times, 2\right.$. Mal als $14^{\mathrm{L}}$ a verzeichnet), 15 $5^{\mathrm{L}}, 16^{\mathrm{L}}, 19,101,103,106,115$, $127,142,285,339^{\mathrm{L}}, 407^{\mathrm{L}}$

$10,171,4,5,9,10,14^{\mathrm{L}}\left(2 \times, 2\right.$. Mal als $14^{\mathrm{L}}$ a verzeichnet), $15^{\mathrm{L}}, 16^{\mathrm{L}}, 19, * 101,103,115,127,285$, $339^{\mathrm{L}}, 407^{\mathrm{L}}$

$10,181,4,5,9,10,14^{\mathrm{L}}\left(2 \times, 2\right.$. Mal als $14^{\mathrm{L}}$ a verzeichnet $), 15^{\mathrm{L}}, 16^{\mathrm{L}}, 19 * * 101,103,115,127,285$, $339^{\mathrm{L}}, 407^{\mathrm{L}}$

$10,19 \quad 1,4,5,9,10,12,16^{\mathrm{L}}, 19,101,103,115,127,285$

$10,20 \quad 1,4,5,9,10,12,16^{\mathrm{L}}, 19,101,103,115,127,285$

$10,21 \quad 1,4,5,9,10,12,16^{\mathrm{L}}, 19,101,103,115,127,142,197,285$

$10,22 \quad 1,4,5,9,10,19,101,103,115,127,142,197,285$

$10,23 \quad 1,4,5,9,10,16^{\mathrm{L}}, 19,101,103,115,127,142,197,285$

$10,24 \quad 1,4,5,9,10,16^{\mathrm{L}}, 19,101,103,115,127,142,197,259,285, * 647$

$10,251,4,5,9,10,16^{\mathrm{L}}, 19,101,103,115,127,142,173,197,259,285,647$

$10,261,4,5,9,10,16^{\mathrm{L}}, 19,101,103,115,127,142,173,197,259,285,647$

$10,27 \quad 1,4,5,9,10,16^{\mathrm{L}}, 19,101,103,115,127,142,173,197,259,285,647$

$10,281,4,5,9,10,16^{\mathrm{L}}, 19,101,103,115,127,142,173,197,259,285,647$

$10,291,4,5,9,10,16^{\mathrm{L}}, 19,101,103,115,127,142,173,197,285,647$

$10,301,4,5,9,10,16^{\mathrm{L}}, 19,103,115,127,142,173,197,285,647$

$10,31 \quad 1,4,5,9,10,16^{\mathrm{L}}, 19,103,115,127,142,173,197,285,294^{\mathrm{L}}, 647$

$10,321,4,5,9,10,16^{\mathrm{L}}, 19,103,115,127,142,173,197,285,294^{\mathrm{L}}$

$10,331,4,5,9,10,12,16^{\mathrm{L}}, 19,81,103,115,127,142,173,197,285,647$

$10,341,4,5,9,10,12,16^{\mathrm{L}}, 19,81,103,115,127,142,173,197,259,285,647$

$10,351,4,5,8,9,10,12,16^{\mathrm{L}}, 19,81,103,115,127,142,173,197,259,285,647$

$10,361,4,5,8,9,10,12,16^{\mathrm{L}}, 19,81,103,115, * 121,127,142,173,197,259,285,647$

$10,371,4,5,8,9,10,16^{\mathrm{L}}, 19,81,82,103,115, * 121,127,142,173,197,259,285,647$

$10,381,4,5,8,9,10,16^{\mathrm{L}}, 19,81,82,103,115,127,142,173,197,259,285$

$10,391,4,5,8,9,10,16^{\mathrm{L}}, 19,81,103,115,127,142,173,197,285$

$10,40 \quad 1,4,5,8,9,16^{\mathrm{L}}, 19,81,103,108,115,127,142,173,197,285$

$10,41 \quad 1,4,5,9,10,16^{\mathrm{L}}, 19,81,103,108,115, * 121,127,142,173,197,285$

$10,421,4,5,9,10,16^{\mathrm{L}}, 19,81,82,103,115,121,127,142,173,197,285$

$11,1 \quad 1,4,5,9,10,19,81,82,103,115,121,127,142,173,197,285$

$11,2 \quad 1,4,5,9,10,19,81,82,103,115,127,142,173,197,285$

$11,3 \quad 1,4,5,9,10,19,81,82,103,108,115,127,142,197,285$

$11,4 \quad 1,4,5,9,10,12,19,81,103,115,127,142,197,285$

$11,5 \quad 1,4,5,9,10,12,19,81,103,115,127,142,197,285,324^{\mathrm{L}}$

$11,6 \quad 1,4,5,9,10,12,19,81,82,103,115,127,142,197,285,324^{\mathrm{L}}$

$11,7 \quad 1,4,5,9,10,19,81,82,103,115,127,197,285,324^{\mathrm{L}}$

$11,8 \quad 1,4,5,9,10,19,81,82,103,115,127,197,285,324^{\mathrm{L}}$

$11,91,4,5,9,10,19,81,82,103,115,127,197,285,324^{\mathrm{L}}$

$11,10 \quad 1,4,5,9,10,19,81,103,108,115,127,197,285$

$11,11 \quad 1,4,5,9,10,19,81,103,108,127,197,285$ 


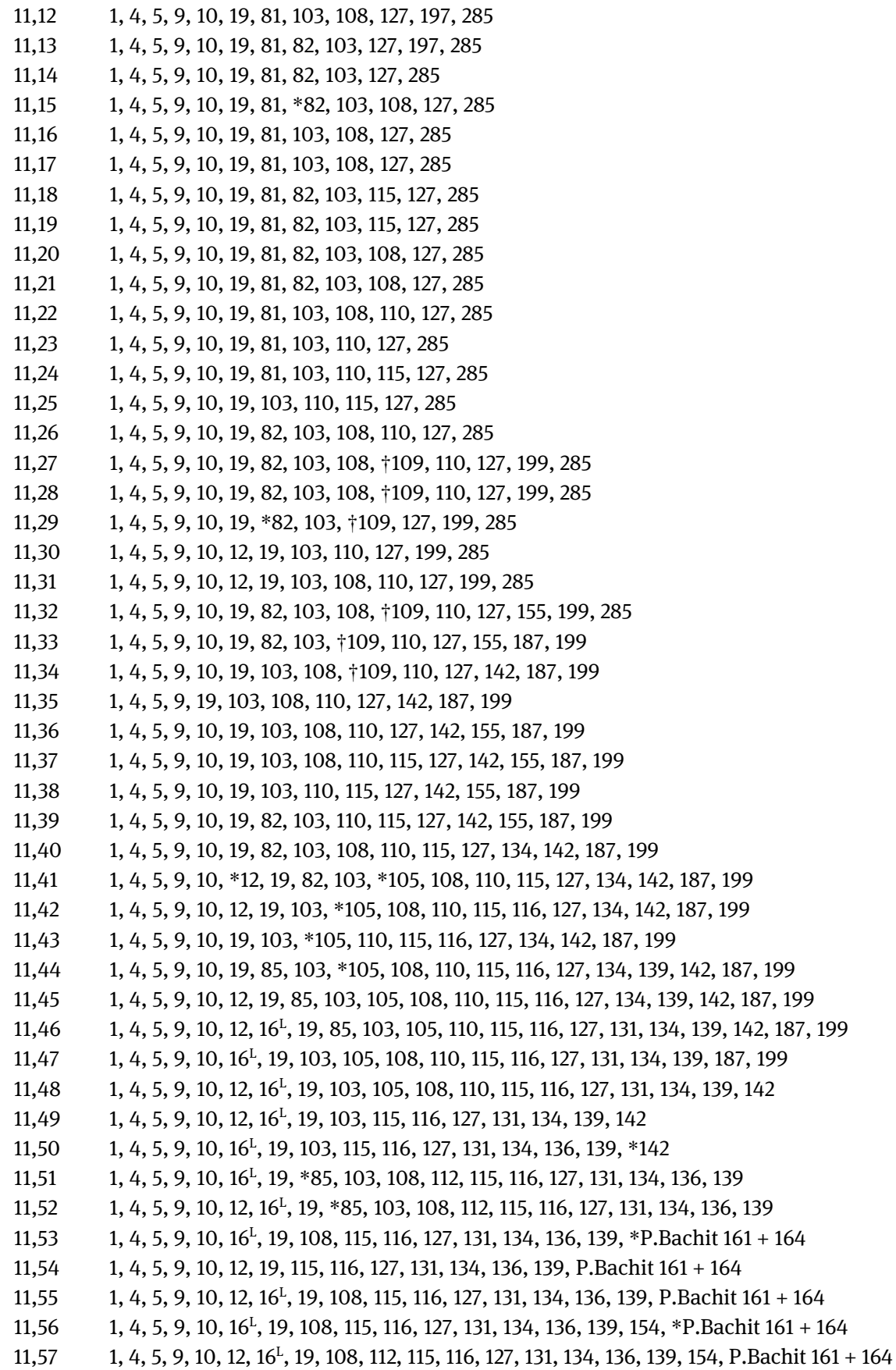


$12,1 \quad 1,4,5,9,10,12,16^{\mathrm{L}}, 19,108,112,116,127,131,134,139,154$, P.Bachit $161+164$

$12,2 \quad 1,4,5,9,10,12,16^{\mathrm{L}}, 19,116,127,131,134,136,139,154$, P.Bachit $161+164$

$12,3 \quad 1,4,5,9,10,12,16^{\mathrm{L}}, 19,116,127,131,134,136,154$

$12,4 \quad 1,4,5,9,10,12,16^{\mathrm{L}}, 19,116,127,131,134,136$

$12,5 \quad 1,4,5,9,10,12,16^{\mathrm{L}}, 19,85,116,127,131,134,154$

$12,6 \quad 1,4,5,7,9,10,16^{\mathrm{L}}, 19,85,112,116,127,131,134,136,154,296^{\mathrm{L}}$

$12,7 \quad 1,4,5,7,9,10,16^{\mathrm{L}}, 19,112,116,127,131,134,136,154,296^{\mathrm{L}}$

$12,8 \quad 1,4,5,7,9,10,12,16^{\mathrm{L}}, 19,116,127,131,134,136,142,296^{\mathrm{L}}$

12,9

12,10

12,11

12,12

12,13

12,14

12,15

12,16

12,17

12,18

12,19

12,20

12,21

12,22

12,23

12,24

$1,4,5,9,10,12,19,116,127,131,134,136,142,154,285$

$1,4,5,9,10,116,127,131,134,136,142,154,285$

$1,4,5,9,10,19,116,127,131,134,136,142,154,285$

$1,4,5,7,9,10,12,14^{\mathrm{L}}, 15^{\mathrm{L}}, 19,112,116,127,131,134,136,142,285,301^{\mathrm{L}}, 440^{\mathrm{L}}$

$1,4,5,7,9,10,12,14^{\mathrm{L}}, 15^{\mathrm{L}}, 19,112,116,127,134,136,142,285,301^{\mathrm{L}}, 440^{\mathrm{L}}$

$1,4,5,9,10,14^{\mathrm{L}}, 15^{\mathrm{L}}, 19,116,127,142,285,301^{\mathrm{L}}, 440^{\mathrm{L}}$

$1,4,5,9,10,14^{\mathrm{L}}, 15^{\mathrm{L}}, 19,85,116,127,142,285,301^{\mathrm{L}}, 440^{\mathrm{L}}$

$1,4,5,7,9,10,12,14^{\mathrm{L}}, 15^{\mathrm{L}}, 19,85,116,127,131,136,142,285,301^{\mathrm{L}}, 440^{\mathrm{L}}$

$1,4,5,7,9,10,12,14^{\mathrm{L}}, 15^{\mathrm{L}}, 19,85,116,127,131,136,142,285,301^{\mathrm{L}}, 440^{\mathrm{L}}$

$1,4,5,7,9,10,14^{\mathrm{L}}, 15^{\mathrm{L}}, 19,127, * 131,136,142,285,301^{\mathrm{L}}, 440^{\mathrm{L}}$

$1,4,5,9,10,14^{\mathrm{L}}, 15^{\mathrm{L}}, 19,105,116,127,136,154,285,301^{\mathrm{L}}, 440^{\mathrm{L}}$

$1,4,5,9,10,14^{\mathrm{L}}, 15^{\mathrm{L}}, 19,105,106,116,127,154,285,301^{\mathrm{L}}, 440^{\mathrm{L}}$

$1,4,5,7,9,10,14^{\mathrm{L}}, 15^{\mathrm{L}}, 19,105,106,116,127,131,136,139,154,285,301^{\mathrm{L}}, 440^{\mathrm{L}}$

$1,4,5,7,9,10,14^{\mathrm{L}}, 15^{\mathrm{L}}, 19,106,116,127,131,136,139,154,285,301^{\mathrm{L}}, 440^{\mathrm{L}}$

$1,4,5,7,9,10,14^{\mathrm{L}}, 15^{\mathrm{L}}, 19,106,116,127,131,136,139,154,173,285,301^{\mathrm{L}}, 440^{\mathrm{L}}$

$1,4,5,9,10,12,14^{\mathrm{L}}, 15^{\mathrm{L}}, 19,106,116,127,131,136,139,154,173,285,440^{\mathrm{L}}, 663^{\mathrm{L}}$ (nur Incipit, Typikon)

$12,25 \quad 1,4,5,9,10,12,14^{\mathrm{L}}, 15^{\mathrm{L}}, 19,106,116,127,136,139,141,154,173,285,440^{\mathrm{L}}$

12,26

$1,4,5,7,9,10,14^{\mathrm{L}}, 15^{\mathrm{L}}, 19,106,116,127,136,139,141,154,173,300^{\mathrm{L}}, 440^{\mathrm{L}}$

12,27

12,28

12,29

12,30

12,31

$1,4,5,7,9,10,14^{\mathrm{L}}, 15^{\mathrm{L}}, 19,106,116,127,131,136,139,141,154,173,300^{\mathrm{L}}, 440^{\mathrm{L}}$

$1,4,5,9,10, * 12,14^{\mathrm{L}}, 15^{\mathrm{L}}, 19,106,127,131,136,139,141,154,173,440^{\mathrm{L}}$

12,32

12,33

12,34

12,35

12,36

12,37

$1,4,5,7,9,10,12,15^{\mathrm{L}}, 19,106,116,127,131,139,141,154,173,396^{\mathrm{L}}, 440^{\mathrm{L}}$

$1,4,5,7,9,10,15^{\mathrm{L}}, 19,106,116,127,136,141,154,173,440^{\mathrm{L}}$

$1,4,5,7,9,10,15^{\mathrm{L}}, 19,106,116,127,136,139,141,154,173,440^{\mathrm{L}}$

$1,4,5,7,9,10,12,15^{\mathrm{L}}, 19,106,116,127,136,139,141,154,173,440^{\mathrm{L}}$

$1,4,5,9,10,12,19,106,116,127,136,139,141,154,173,440^{\mathrm{L}}$

$1,4,5,9,10,12,19,106,116,127,136,139,141,173,440^{\mathrm{L}}$

$1,4,5,7,9,10,16^{\mathrm{L}}, 19,106,116,127,139,141,173,305^{\mathrm{L}}, 440^{\mathrm{L}}$

$1,4,5,9,10,12,16^{\mathrm{L}}, 19,105,106,116,127,136,139,142,173,182,305^{\mathrm{L}}, 440^{\mathrm{L}}$

12,38

$1,4,5,9,10,16^{\mathrm{L}}, 19,105,106,116,127,139,142,173,182,305^{\mathrm{L}}$

12,39

$1,4,5,7,9,10,16^{\mathrm{L}}, 19,103,105,106,116,127,139,173,182,305^{\mathrm{L}}$

12,40

$1,4,5,7,9,10,16^{\mathrm{L}}, 19,101,103,105,106,116,127,173,182,305^{\mathrm{L}}$

12,41

12,42

$1,4,5,7,9,10,12,16^{\mathrm{L}}, 19,101,103,105,106,116,127,136,154,182,305^{\mathrm{L}}$

12,43

$1,4,5,9,10,16^{\mathrm{L}}, 19,101,103,105,106,116,127,136,142,154,182,305^{\mathrm{L}}$

12,44

$1,4,5,7,9,10,12,16^{\mathrm{L}}, 19,101,103,105,106,116,127,142,154,182,305^{\mathrm{L}}$

12,45

$1,4,5,7,9,10,12,16^{\mathrm{L}}, 19,101,103,105,106,116,127,154,182$

$1,4,5,7,9,10,12,19,103,105,106,116,127,136,154,182$

12,46

1, 4, 5, 7, 9, 10, 103, 105, 106, 116, 127, *136, 154, 182

$1,4,5,9,10,19,103,105,106,116,127,136,154,182$ 
$12,47 \quad 1,4,5,7,9,10,19,101,103,106,116,127,154,182$

$12,48 \quad 1,4,5,7,9,10,19,101,103,106,116,126,127,132,141,154,182$

$12,49 \quad 1,4,5,7,9,10,19,103,106,116,126,127,132,141,154,182$

$12,501,4,5,9,10,19,101,103,106,116,126,127,132,141,154,182$

$13,1 \quad 1,4,5,7,9,10,16^{\mathrm{L}}, 19,101,103,106,116,126,127,132,141,154,182$

$13,2 \quad 1,4,5,7,9,10,16^{\mathrm{L}}, 19,103,106,108,116,126,132,141,154,179,182,243$

$13,3 \quad 1,4,5,9,10,16^{\mathrm{L}}, 19,101,103,106,108,126,132,141,179,180,243$

$13,4 \quad 1,4,5,9,10,16^{\mathrm{L}}, 19,101,103,106,108,126,132,141,180$

$13,5 \quad 1,4,5,7,9,10,16^{\mathrm{L}}, 19,57,101,103,106,108,126,132,139,141,180,243$

$13,6 \quad 1,4,5,7,9,10,16^{\mathrm{L}}, 19,57,103,106,108,126,132,139,141,180,243$

$13,7 \quad 1,4,5,7,9,10,16^{\mathrm{L}}, 19,57,103,106,108,126,132,139,141,142,180,243$

$13,8 \quad 1,4,5,9,10,16^{\mathrm{L}}, 19,57,103,106,108,126,132,139,141,142,179,180$

$13,91,4,5,9,10,16^{\mathrm{L}}, 19,57,103,108,126,139,141,142,179$

$13,101,4,5,7,9,10,16^{\mathrm{L}}, 19,57,103,108,126,139,142,286$

$13,11 \quad 1,4,5,7,9,10,16^{\mathrm{L}}, 19,57,103,108,126,139,142,286$

$13,121,4,5,9,10,16^{\mathrm{L}}, 19,57,103,108,126,139,142,286$

$13,131,4,5,9,10,16^{\mathrm{L}}, 19,57,103,108,126,139,142,266,286$

$13,141,4,5,7,9,10,16^{\mathrm{L}}, 19,103,108,126,139,142,266,286$

$13,151,4,5,7,9,10,16^{\mathrm{L}}, 19,57,103,108,126,139,142,266,286$

$13,161,4,5,7,9,10,16^{\mathrm{L}}, 19,57,103,108,126,139,142,266$

$13,17 \quad 1,4,5,9,10,16^{\mathrm{L}}, 19,57,103,108,126,139,142,154,266$

$13,181,4,5,9,10,16^{\mathrm{L}}, 19,57,103,108,121,139,142,154,266$

$13,19 \quad 1,4,5,7,9,10,16^{\mathrm{L}}, 19,57,103,108,121,139,142,154,266$

$13,201,4,5,7,9,10,16^{\mathrm{L}}, 19,57,103,108,121,124,139,142,154,266$

$13,21 \quad 1,4,5, * 7,9,10,16^{\mathrm{L}}, 19,57,103,108,124,139,142,154,266,349^{\mathrm{L}}$

$13,22 \quad 1,4,5,9,10,16^{\mathrm{L}}, 19,57,103,108,121,124,139,142,154,266,349^{\mathrm{L}}$

$13,231,4,5,7,9,10,16^{\mathrm{L}}, 19,57,103,108,121,124,139,142,154,266,349^{\mathrm{L}}$

$13,241,4,5,7,9,10,16^{\mathrm{L}}, 19,57,103,108,121,124,139,142,154,266,349^{\mathrm{L}}$

$13,251,4,5,7,9,10,16^{\mathrm{L}}, 19,57,103,108,121,124,139,142,154,266,349^{\mathrm{L}}$

$13,261,4,5,9,10,16^{\mathrm{L}}, 19,57,103,108,121,124,139,142,154,266,349^{\mathrm{L}}$

$13,27 \quad 1,4,5,9,10,16^{\mathrm{L}}, 19,57,103,108,124,139,142,154,266,349^{\mathrm{L}}$

$13,28 \quad 1,4,5,7,9,10,16^{\mathrm{L}}, 19,57,103,108,124,139,142,154,266,349^{\mathrm{L}}$

$13,291,4,5,7,9,10,16^{\mathrm{L}}, 19,57,82,89,103,108,121,124,139,142,154,180,266,349^{\mathrm{L}}$

$13,301,4,5,7,9,10,16^{\mathrm{L}}, 19,57,82,89,103,108,121,124,139,142,154,180,266,349^{\mathrm{L}}$

$13,31 \quad 1,4,5,9,10,19,57,82,103,108,121,124,139,142,154,266$

$13,32 \quad 1,4,5,9,10,19,57,108,124,139,142,180,266$

$13,331,4,5,7, * 8,9,10,16^{\mathrm{L}}, 19,57,89,108,121,124,139,142,180$

$13,341,4,5,7, * 8,9,10,16^{\mathrm{L}}, 19,57,108,121,124,139,142,180$

$13,35 \quad 1,4,5,9,10,16^{\mathrm{L}}, 19,57,108,121,124,142$

$13,36 \quad 1,4,5,8,9,10,16^{\mathrm{L}}, 19,89,108,121,124,139,142$

$13,37 \quad 1,4,5,9,10,12,16^{\mathrm{L}}, 19,108,124,139,142$

$13,381,4,5,9,10,12,16^{\mathrm{L}}, 19,89,108,124,139,142, * 185$

$14,1 \quad 1,4,5,9,10,16^{\mathrm{L}}, 19,89,108,124,139,142, * 185$

$14,2 \quad 1,4,5,9,10,16^{\mathrm{L}}, 19,89,108,124,139,142, * 185$

$14,3 \quad 1,4,5,9,10, * 12,16^{\mathrm{L}}, 19,108,139,142$

$14,4 \quad 1,4,5,9,10,16^{\mathrm{L}}, 19,108,139,142$ 


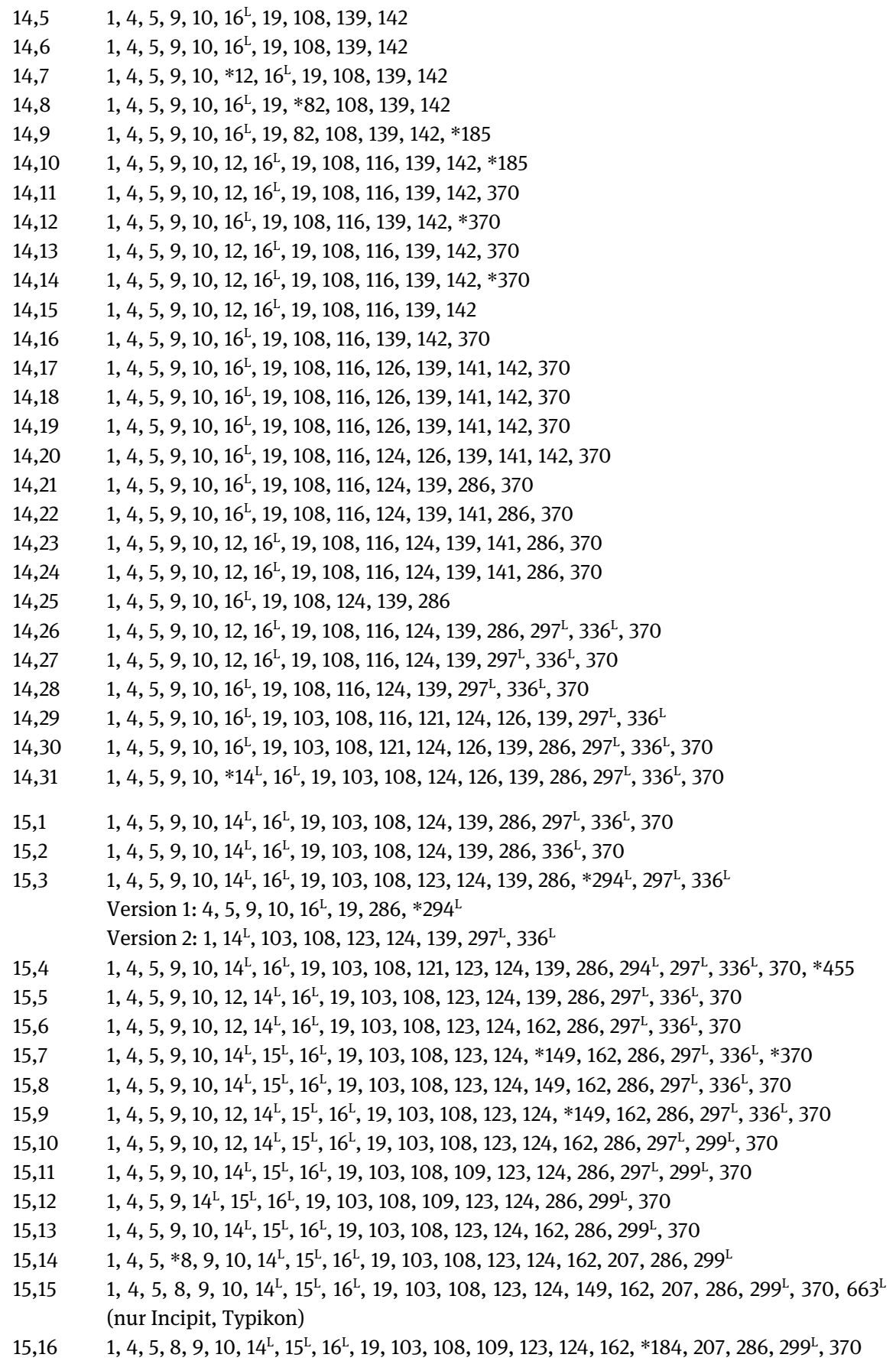


$15,17 \quad 1,4,5,8,9,10,15^{\mathrm{L}}, 16^{\mathrm{L}}, 19,103,108,109,123,124, * 184,207,286,299^{\mathrm{L}}$

$15,181,4,5,8,9,10,16^{\mathrm{L}}, 19,103,108,123,124,162,184,207,286,299^{\mathrm{L}}, * 370$

$15,191,4,5,8,9,10,12,16^{\mathrm{L}}, 19,103,108,123,124,162,184,207,286,299^{\mathrm{L}}, 370$

$15,201,4,5,7,8,9,10,16^{\mathrm{L}}, 19,103,108,123,124,162,184,207,286,299^{\mathrm{L}}, 370$

$15,21 \quad 1,4,5,7,8,9,10,16^{\mathrm{L}}, 19,103,123,124, * 184,207,286,299^{\mathrm{L}}, 370$

$15,221,4,5,7,8,9,10,16^{\mathrm{L}}, 19,103,123,124,139,162,207,286,299^{\mathrm{L}}, 370$

$15,23 \quad 1,4,5,7,9,10,16^{\mathrm{L}}, 19,103,123,124,139,162,286,299^{\mathrm{L}}, * 370$

$15,241,4,5,7,9,10,16^{\mathrm{L}}, 19,103,123,124,139,162,184,207,286,299^{\mathrm{L}}, 370$

$15,25 \quad 1,4,5,7,9,10,16^{\mathrm{L}}, 19,82,103,123,124,139,162,184,207,286,299^{\mathrm{L}}, 370$

$15,261,4,5,7,9,10,14^{\mathrm{L}}, 15^{\mathrm{L}}, 16^{\mathrm{L}}, 19,103,123,124,139,162,207,286,297^{\mathrm{L}}, 299^{\mathrm{L}}, 370$

$15,271,4,5,7,9,10,14^{\mathrm{L}}, 15^{\mathrm{L}}, 16^{\mathrm{L}}, 19,103,123,124,139,184,275,286,297^{\mathrm{L}}, 370$

$16,11,4,5,7,9,10,14^{\mathrm{L}}, 15^{\mathrm{L}}, 16^{\mathrm{L}}, 19,103,123,124,139,141,184,275,286,297^{\mathrm{L}}, 299^{\mathrm{L}}, 370$

$16,21,4,5,7,9,10,14^{\mathrm{L}}, 15^{\mathrm{L}}, 16^{\mathrm{L}}, 19,103,106,109,123,124,139,141,184,275,286,297^{\mathrm{L}}$, $299^{\mathrm{L}}, 370$

$16,31,4,5,7,9,10,14^{\mathrm{L}}, 15^{\mathrm{L}}, 16^{\mathrm{L}}, 19,103,106,109,123,124,139,141, * 184,275,286,299^{\mathrm{L}}$, 370

$16,41,4,5,7,9,10,14^{\mathrm{L}}, 15^{\mathrm{L}}, 16^{\mathrm{L}}, 19,82,103,106,109, * 121,123,124,139,141,184,286$, $297^{\mathrm{L}}, 299^{\mathrm{L}}, 370$

$16,51,4,5,7,9,10,14^{\mathrm{L}}, 15^{\mathrm{L}}, 16^{\mathrm{L}}, 19,82,103,106,109,121,123,124,139,141,184,286,297^{\mathrm{L}}$, $299^{\mathrm{L}}, 370$

$16,61,4,5,7,9,10,14^{\mathrm{L}}, 15^{\mathrm{L}}, 16^{\mathrm{L}}, 19,103,106,109,121,123,124,139,141,184,286,297^{\mathrm{L}}$, $299^{\mathrm{L}}, 370$

$16,71,4,5,7,9,10,14^{\mathrm{L}}, 15^{\mathrm{L}}, 16^{\mathrm{L}}, 19,103,106,121,123,124,139,141,184,275,286,297^{\mathrm{L}}$, $299^{\mathrm{L}}, 370$

$16,81,4,5,7,9,10,14^{\mathrm{L}}, 15^{\mathrm{L}}, 16^{\mathrm{L}}, 19,103,106,121,123,124,139,141,184,275,286,297^{\mathrm{L}}$, $299^{\mathrm{L}}, 370$

$16,91,4,5,7,9,10,14^{\mathrm{L}}, 15^{\mathrm{L}}, 16^{\mathrm{L}}, 19,103,106,109, * 121,123,124,139,141,286,297^{\mathrm{L}}, 370$

$16,101,4,5,7,9,10,14^{\mathrm{L}}, 15^{\mathrm{L}}, 16^{\mathrm{L}}, 19,103,106,108,109,121,123,124,139,141,286,370$

$16,111,4,5,7,9,10,14^{\mathrm{L}}, 15^{\mathrm{L}}, 16^{\mathrm{L}}, 19,106,108,109,121,123,124,139,141,286,370$

$16,121,4,5,7,9,10,14^{\mathrm{L}}, 15^{\mathrm{L}}, 16^{\mathrm{L}}, 19,108,109, * 121,123,124,139,141,286,370$

$16,131,4,5,7,9,10,14^{\mathrm{L}}, 15^{\mathrm{L}}, 16^{\mathrm{L}}, 19,106,108,109,123,124,139,141, * 184,286,297^{\mathrm{L}}, 370$

$16,141,4,5,7,9,14^{\mathrm{L}}, 16^{\mathrm{L}}, 19,108,123,124,139,141,297^{\mathrm{L}}, 370$

$16,151,4,5,7,9,10,14^{\mathrm{L}}, 16^{\mathrm{L}}, 19,108,121,123,124,139,141,154,275,297^{\mathrm{L}}, 370$

$16,161,4,5,7,9,10,16^{\mathrm{L}}, 19,108,121,123,124,139,141,154, * 184,275,370$

$16,17 \quad 1,4,5,7,9,10,16^{\mathrm{L}}, 19,108,121,123,124,141,154,370$

$16,181,4,5,7,9,10,16^{\mathrm{L}}, 19,108, * 121,124,141,154,370$

$16,191,4,5,7,9,10,16^{\mathrm{L}}, 19,108, * 121,124,141,154,275,297^{\mathrm{L}}, 370$

$16,201,4,5,7,9,10,16^{\mathrm{L}}, 19,59^{\mathrm{L}}, 106,108,124,141,154,275,370$

$16,21 \quad 1,4,5,9,10,16^{\mathrm{L}}, 19,59^{\mathrm{L}}, 106,108, * 121,124,141,154,275,370$

$16,221,4,5,9,10,16^{\mathrm{L}}, 19,59^{\mathrm{L}}, 106,108,115,124,141,154$

$16,23 \quad 1,4,5,9,10,16^{\mathrm{L}}, 19,59^{\mathrm{L}}, 106,108,115,124,141,154$

$16,24 \quad 1,4,5,9,10,16^{\mathrm{L}}, 19,87,108,115,124,141,154, * 370$

$16,25 \quad 1,4,5,9,10,16^{\mathrm{L}}, 19,82,87,108,115,124,154,370$

$16,26 \quad 1,4,5,9,10,16^{\mathrm{L}}, 19,82,108,115,124,154$

$16,27 \quad 1,4,5,9,10,16^{\mathrm{L}}, 19,108,115,124,154$, *No. 931

$16,281,4,5,9,10,16^{\mathrm{L}}, 19,105,108,115,124,154$, No. 931 
$16,291,4,5,9,10,16^{\mathrm{L}}, 19,87,103,105,108,115,124,154$, No. 931

$16,301,4,5,9,10,16^{\mathrm{L}}, 19, * 82,87,103,108,115,124,154, * 370$

$16,31 \quad 1,4,5,9,10,16^{\mathrm{L}}, 19,82,87,102,103,108,115,124,154,370$

$16,321,4,5,9,10,16^{\mathrm{L}}, 19,82,102,103,108,115,124,131,154,370$, No. 931

$16,331,4,5,9,10,16^{\mathrm{L}}, 19,103,108,115,124,131,154,370$

$17,1 \quad 1,4,5,9,10,15^{\mathrm{L}}, 16^{\mathrm{L}}, 19,87,103,108,115,124,131,154,370$, No. 931

$17,2 \quad 1,4,5,9,10,15^{\mathrm{L}}, 16^{\mathrm{L}}, 19,87,102,103,108,115,124, * 131,370$

$17,3 \quad 1,4,5,9,10,15^{\mathrm{L}}, 16^{\mathrm{L}}, 19,87,102,103,108,115,124,131,140,154,370$

$17,41,4,5,9,10,14^{\mathrm{L}}, 15^{\mathrm{L}}, 16^{\mathrm{L}}, 19,103,108,115,124,131,140,154,302^{\mathrm{L}}, 370$

$17,51,4,5,9,10,14^{\mathrm{L}}, 15^{\mathrm{L}}, 16^{\mathrm{L}}, 19,103,108,115,124, * 131,140,154,302^{\mathrm{L}}, 370$, No. 931

$17,61,4,5,9,10,14^{\mathrm{L}}, 15^{\mathrm{L}}, 16^{\mathrm{L}}, 19,103,108,115,124, * 131,140,154,292^{\mathrm{L}}, 302^{\mathrm{L}}, 370$, No. 931

$17,71,4,5,9,10,14^{\mathrm{L}}, 15^{\mathrm{L}}, 16^{\mathrm{L}}, 19,102,103,108,115,124, * 131,140,292^{\mathrm{L}}, 302^{\mathrm{L}}, 370$

$17,81,4,5,9,10,14^{\mathrm{L}}, 15^{\mathrm{L}}, 16^{\mathrm{L}}, 19,22,74,87,102,103,108,115,124,131,140,292^{\mathrm{L}}, 297^{\mathrm{L}}$, $302^{\mathrm{L}}, 370$

$17,91,4,5,9,10,14^{\mathrm{L}}, 15^{\mathrm{L}}, 16^{\mathrm{L}}, 19,74,87,103,108,124,131,140,292^{\mathrm{L}}, 297^{\mathrm{L}}, 302^{\mathrm{L}}, 370$

$17,101,4,5,9,10,14^{\mathrm{L}}, 15^{\mathrm{L}}, 16^{\mathrm{L}}, 19,74,103,108,124,131,140,292^{\mathrm{L}}, 302^{\mathrm{L}}, 370$

$17,11 \quad 1,4,5,9,10,14^{\mathrm{L}}, 15^{\mathrm{L}}, 16^{\mathrm{L}}, 19,74,102,103,108,124,127,131,140,142,292^{\mathrm{L}}, 370$

$17,121,4,5,9,10,14^{\mathrm{L}}, 16^{\mathrm{L}}, 19,74,103,108,124,127,131,140,142,292^{\mathrm{L}}, * 370$

$17,131,4,5,9,10,14^{\mathrm{L}}, 16^{\mathrm{L}}, 19,74,103,108,124,126,131,140,142,292^{\mathrm{L}}, 370$

$17,141,4,5,9,10,14^{\mathrm{L}}, 16^{\mathrm{L}}, 19,74,103,108,124,126,131,140,142,292^{\mathrm{L}}, 370$

17,15

17,16

17,17 $1,4,5,9,10,14^{\mathrm{L}}, 16^{\mathrm{L}}, 19,74, * 82,103,108,124,126,131,140,142,292^{\mathrm{L}}, 370$ $1,4,5,9,10,14^{\mathrm{L}}, 16^{\mathrm{L}}, 19,82,108,124,131,140,142,292^{\mathrm{L}}$

$1,4,5,9,14^{\mathrm{L}}, 16^{\mathrm{L}}, 19,74,82,108,124,131,140,142,292^{\mathrm{L}}, 349^{\mathrm{L}}$

17,19

$1,4,5,9,16^{\mathrm{L}}, 19,74, * 82,108,124,126,131,140,142,292^{\mathrm{L}}, 349^{\mathrm{L}}, 370$

17,20

$1,4,5,9,10,16^{\mathrm{L}}, 19,74,108,124,126, * 131,140,142,209,292^{\mathrm{L}}, 349^{\mathrm{L}}, * 370$

17,21

$1,4,5,9,10,16^{\mathrm{L}}, 19,74,108,124,126,140,142,209,292^{\mathrm{L}}, 349^{\mathrm{L}}, * 370$

17,22

17,23

17,24 $1,4,5,8,9,10,16^{\mathrm{L}}, 19,74,108,124,126,140,209,292^{\mathrm{L}}, 349^{\mathrm{L}}$

$1,4,5,8,9,16^{\mathrm{L}}, 19,22,74, * 82,108,124,126,140,209,292^{\mathrm{L}}, 349^{\mathrm{L}}$

17,25 $1,4,5, * 8,9,10,16^{\mathrm{L}}, 19,74,82,108,124,126,140,209,292^{\mathrm{L}}, 349^{\mathrm{L}}$

17,26 $1,4,5, * 8,9,10,16^{\mathrm{L}}, 19,74,108,124,126,140,292^{\mathrm{L}}, 349^{\mathrm{L}}$ $1,4,5,9,10,16^{\mathrm{L}}, 19,74,108,124,126,140,292^{\mathrm{L}}, 349^{\mathrm{L}}$

18,1 $1,4,5,9,10,16^{\mathrm{L}}, 19,74,108,124,126,140,292^{\mathrm{L}}, 349^{\mathrm{L}}$

18,2 $1,4,5,9,10,16^{\mathrm{L}}, 19,74,108,124,126,140,292^{\mathrm{L}}$

18,3 $1,4,5,9,10,16^{\mathrm{L}}, 19,108,124,140,292^{\mathrm{L}}$

18,4 $1,4,5,9,10,16^{\mathrm{L}}, 19,74,108,124,127,140,235$

18,5 $1,4,5,9,10,16^{\mathrm{L}}, 19,74,108,124,127,140,235$

18,6 $1,4,5,9,10,16^{\mathrm{L}}, 19,74,108,124,127,140,235$

18,7 $1,4,5,8,9,10,16^{\mathrm{L}}, 19,74,108,124,127,140,235,292^{\mathrm{L}}$

$18,81,4,5,8,9,10,16^{\mathrm{L}}, 19,74,108,124,127,140,235,260,292^{\mathrm{L}}, 370,453$ (Zitat in Homilie oder ähnlichem)

$18,9 \quad 1,4,5,8,9,10,16^{\mathrm{L}}, 19,74,108,124,127,140,235,260,292^{\mathrm{L}}, 370$

$18,101,4,5,8,9,10,16^{\mathrm{L}}, 19,74,108,124,127,140,235,260,292^{\mathrm{L}}, 370$

$18,11 \quad 1,4,5,8,9,10,16^{\mathrm{L}}, 19,74,108,124,127,140,235,260, * 370$

$18,12 \quad 1,4,5,8,9,10,16^{\mathrm{L}}, 19,74,108,124,127,140,141,235,260$

$18,13 \quad 1,4,5,8,9,10,16^{\mathrm{L}}, 19,74,108,124,127,140,141,235,260$ 
$18,14 \quad 1,4,5,8,9,10,16^{\mathrm{L}}, 19,74,108,124,127,140,141,235,260$

$18,151,4,5,8,9,10,16^{\mathrm{L}}, 19,108,124,127,140,141,235,260,292^{\mathrm{L}}, 370$

$18,161,4,5,9,10,16^{\mathrm{L}}, 19,108,127,140,141,235,260,292^{\mathrm{L}}$

$18,17 \quad 1,4,5,9,10,16^{\mathrm{L}}, 19,108,127,140,141,235,260,292^{\mathrm{L}}$

$18,18 \quad 1,4,5,9,10,16^{\mathrm{L}}, 19,108,124,127,140,141,235,260,292^{\mathrm{L}}$

$18,191,4,5,9,10,16^{\mathrm{L}}, 19,108,124,127,140,141,235,260,292^{\mathrm{L}}, 370$

$18,20 \quad 1,4,5,9,10,16^{\mathrm{L}}, 19,108,127,140,141,235,260,292^{\mathrm{L}}, 370$

$18,21 \quad 1,4,5,9,10,16^{\mathrm{L}}, 19,82,108,127,140,141,235,260,292^{\mathrm{L}}$

$18,22 \quad 1,4,5,9,10,16^{\mathrm{L}}, 19,82,108,127,140,141,235,260,292^{\mathrm{L}}$

$18,23 \quad 1,4,5,9,10,16^{\mathrm{L}}, 19,82,103,108,127,140,235,260,292^{\mathrm{L}}, 370$

$18,241,4,5,9,16^{\mathrm{L}}, 19,103,108,127,140,235,260,292^{\mathrm{L}}, 370$

$18,25 \quad 1,4,5,9,10,16^{\mathrm{L}}, 19,103,108,127,140,235,260,292^{\mathrm{L}}$

$18,261,4,5,9,10,16^{\mathrm{L}}, 19,103,108,127,140,205,235,260,292^{\mathrm{L}}$

$18,27 \quad 1,4,5,9,10,16^{\mathrm{L}}, 19,103,108,127,140,205,260,292^{\mathrm{L}}$

$18,28 \quad 1,4,5,9,10,16^{\mathrm{L}}, 19,83,103,108,127,140,205,260,292^{\mathrm{L}}$

$18,291,4,5,9,10,16^{\mathrm{L}}, 19,83,103,108,127,139,140,260,292^{\mathrm{L}}$

$18,301,4,5,9,10,16^{\mathrm{L}}, 19,83,103,108,127,139,140,260,292^{\mathrm{L}}$

$18,31 \quad 1,4,5,9,10,16^{\mathrm{L}}, 19,83,103,108,124,127,139,140,155,260,292^{\mathrm{L}}$

$18,321,4,5,9,10,16^{\mathrm{L}}, 19,83,103,108,124,127,139,140,155,260,292^{\mathrm{L}}$

$18,331,4,5,9,10,16^{\mathrm{L}}, 19,103,108,124,127,139,140,155,260,292^{\mathrm{L}}$

$18,34 \quad 1,4,5,9,10,16^{\mathrm{L}}, 19,103,108,124,127,139,140,155,260,292^{\mathrm{L}}$

$18,351,4,5,9,10,16^{\mathrm{L}}, 19,83,103,108,124,127,139,140,155,260,292^{\mathrm{L}}$

$18,361,4,5,9,10,16^{\mathrm{L}}, 19,82,83,103,108,116,124,127,139,140,142,155,260,292^{\mathrm{L}}, 453$ (Zitat in Homilie oder ähnlichem)

$18,37 \quad 1,4,5,9,10,16^{\mathrm{L}}, 19,83,103,108,116,124,127,139,140,142,155,260,292^{\mathrm{L}}, 330^{\mathrm{L}}$

$18,381,4,5,9,10,16^{\mathrm{L}}, 19,83,103,108,110,116,124,127,139,140,155,260,292^{\mathrm{L}}$

$18,391,4,5,9,10,16^{\mathrm{L}}, 19,103,108,110,116,124,127,139,140,155,292^{\mathrm{L}}$

$18,401,4,5,9,10,16^{\mathrm{L}}, 19,103,108,110,116,124,127,139,140,141,142,155,292^{\mathrm{L}}$

$19,111,4,5,9,10,16^{\mathrm{L}}, 19,103,108,116,124,127,139,140,141,142,155,292^{\mathrm{L}}, 298^{\mathrm{L}}, 663^{\mathrm{L}}$ (nur Incipit, Typikon)

$19,21,4,5,9,10,16^{\mathrm{L}}, 19,103,108,116,124,127,139,140,141,142,155,292^{\mathrm{L}}, 298^{\mathrm{L}}$

$19,31,4,5,9,10,16^{\mathrm{L}}, 19,103,108,116,124,127,139,140,141,142,155,292^{\mathrm{L}}, 298^{\mathrm{L}}$

$19,41,4,5,9,10,16^{\mathrm{L}}, 19,103,108,110,116,124,127,140,141,142,155,292^{\mathrm{L}}, 298^{\mathrm{L}}$

$19,5 \quad 1,4,5,9,10,16^{\mathrm{L}}, 19,103,108,110,116,124,127,140,141,292^{\mathrm{L}}, 298^{\mathrm{L}}$

$19,61,4,5,9,10,16^{\mathrm{L}}, 19,103,108,110,116,124,127,140,141,292^{\mathrm{L}}, 298^{\mathrm{L}}$

$19,7 \quad 1,4,5,9,10,16^{\mathrm{L}}, 19,58^{\mathrm{L}}, 103,108,116,124,127,140,141,292^{\mathrm{L}}, 298^{\mathrm{L}}$

$19,81,4,5,9,10,16^{\mathrm{L}}, 19,58^{\mathrm{L}}, 103,108,116,124,127,140,141,292^{\mathrm{L}}, 298^{\mathrm{L}}$

$19,91,4,5,9,10,16^{\mathrm{L}}, 19,58^{\mathrm{L}}, 103,108,116,124,127,140,141,292^{\mathrm{L}}, 298^{\mathrm{L}}$

$19,101,4,5,9,10,16^{\mathrm{L}}, 19,58^{\mathrm{L}}, 103,108,116,124,127,140,141,205,292^{\mathrm{L}}, 298^{\mathrm{L}}$

$19,11 \quad 1,4,5,9,10,16^{\mathrm{L}}, 19,58^{\mathrm{L}}, 103,108,116,124,127,140,141,205,292^{\mathrm{L}}, 298^{\mathrm{L}}$

$19,121,4,5,9,10,16^{\mathrm{L}}, 19,58^{\mathrm{L}}, 103,108,116,124,127,140,292^{\mathrm{L}}, 298^{\mathrm{L}}$

$19,13 \quad 1,4,5,9,10,16^{\mathrm{L}}, 19,58^{\mathrm{L}}, 103,108,116,124,127,140,298^{\mathrm{L}}$

19,14 1, 4, 5, 9, 10, 16 1 , 19, 58 $, 103,108$ (Schriftduktus des Kopisten bricht in der Kolumne mit 19,14 ab und eine andere Hand fährt mit 19,15 fort), 116, 124, 127, 140, 298 ${ }^{\mathrm{L}}$

$19,151,4,5,9,16^{\mathrm{L}}, 19,58^{\mathrm{L}}, 103,108$ (Text von anderer Hand), 116, 124, 127, 140, $298^{\mathrm{L}}$ 
19,16 1, 4, 5, 9, 10, 16 $, 19,58^{\mathrm{L}}, 103,108$ (Text von anderer Hand, mit Seitenwechsel führt der ursprüngliche Schreiber ab мммтеєвраıос fort), 116, 124, 127, 140, 298 $, 337^{\mathrm{L}}, 352$, $663^{\mathrm{L}}$ (Versincipit als Desinit der Perikope 19,1-16, Typikon)

$19,17 \quad 1,4,5,9,10,16^{\mathrm{L}}, 19,58^{\mathrm{L}}, 103,108,116,124,127,140,155,337^{\mathrm{L}}, 352$

$19,181,4,5,9,10,16^{\mathrm{L}}, 19, * 82,103,108,110,116,121,124,127,140,155,199,337^{\mathrm{L}}$

$19,191,4,5,9,10,14^{\mathrm{L}}, 16^{\mathrm{L}}, 19,82,103,108,110,116,121,124,127,140,155,199,337^{\mathrm{L}}$

$19,201,4,5,9,10,14^{\mathrm{L}}, 16^{\mathrm{L}}, 19,82,103,108,110,116,121,124,127,140,155,199,335^{\mathrm{L}}, 337^{\mathrm{L}}$, 352

$19,2111,4,5,9,10,16^{\mathrm{L}}, 19,103,108,110,116,121,124,127,140,155,199,335^{\mathrm{L}}, 337^{\mathrm{L}}, 352$

19,22

19,23

19,24 $1,4,5,9,10,14^{\mathrm{L}}, 16^{\mathrm{L}}, 19,103,108,110,116,121,124,127,140,155,199,337^{\mathrm{L}}$

19,25 $1,4,5,9,10,14^{\mathrm{L}}, 16^{\mathrm{L}}, 19,103,108,110,116,121,124,127,140,155,199,335^{\mathrm{L}}, 337^{\mathrm{L}}$ $1,4,5,9,10,16^{\mathrm{L}}, 19,103,108,110,116,121,124,127,142,155,199,335^{\mathrm{L}}, 337^{\mathrm{L}}$ $1,4,5,9,10, * 14^{\mathrm{L}}, 15^{\mathrm{L}}, 16^{\mathrm{L}}, 19,103,108,110,116,121,127,142,155,199,408^{\mathrm{L}}$, No. 948

19,27 $1,4,5,9, * 14^{\mathrm{L}}, 15^{\mathrm{L}}, 16^{\mathrm{L}}, 19,103,108,110,116,121,127,142,155,199,408^{\mathrm{L}}$, No. 948

19,28

19,29

19,30 $1,4,5,9,10,14^{\mathrm{L}}, 15^{\mathrm{L}}, 16^{\mathrm{L}}, 19,103,108,110,116,121,127,142,155,199,408^{\mathrm{L}}$

19,31

19,32 $1,4,5,9,10,15^{\mathrm{L}}, 16^{\mathrm{L}}, 19,103,108,116,121,127,155,199$

19,33

19,34

19,35

19,36

19,37 $1,4,5,9,10,15^{\mathrm{L}}, 16^{\mathrm{L}}, 19,103,108,116,121,127,142,155,199$

19,38 $1,4,5,9,15^{\mathrm{L}}, 16^{\mathrm{L}}, 19,103,108,116,121,127,142,155,199$ $1,4,5,9,10,15^{\mathrm{L}}, 16^{\mathrm{L}}, 19,103,108,116,121,127,142,155,199$, *No. 948

19,40

19,41

19,42 $1,4,5,9,10,15^{\mathrm{L}}, 16^{\mathrm{L}}, 19, * 82,103,108,121,127,155,199$

$20,1 \quad 1,4,5,9,10,14^{\mathrm{L}}, 15^{\mathrm{L}}, 19,103,121,139,155,182,313^{\mathrm{L}}, 335^{\mathrm{L}}, 340^{\mathrm{L}}$, No. 948

$20,2 \quad 1,4,5,9,10,14^{\mathrm{L}}, 15^{\mathrm{L}}, 19,103,116,139,155,168,182,308^{\mathrm{L}}, 313^{\mathrm{L}}$

$20,3 \quad 1,4,5,9,10,14^{\mathrm{L}}, 15^{\mathrm{L}}, 19,103,116,139,168,182,308^{\mathrm{L}}, 335^{\mathrm{L}}, 649$

20,4

20,5

20,6 $1,4,5,9,10,15^{\mathrm{L}}, 16^{\mathrm{L}}, 19,82,103,108,121,127,155,199$ $1,4,5,9,10,15^{\mathrm{L}}, 16^{\mathrm{L}}, 19,82,103,108,121,127,155,199$ $1,4,5,9,10,15^{\mathrm{L}}, 16^{\mathrm{L}}, 19,103,108,121,127,139,155,199$ $1,4,5,9,10,15^{\mathrm{L}}, 16^{\mathrm{L}}, 19,103,108,127,139,155,199$ $1,4,5,9,10,15^{\mathrm{L}}, 16^{\mathrm{L}}, 19,103,108,127,155$ $1,4,5,9,10,16^{\mathrm{L}}, 19,103,108,127,155,182$ $1,4,5,9,10,16^{\mathrm{L}}, 19,103,127,139,155,182$ $1,4,5,9,10,16^{\mathrm{L}}, 19,103,116,127,139,155,182,217$ $1,4,5,9,10,16^{\mathrm{L}}, 19,103,116,139,155,182,217$, No. 948 $1,4,5,9,10,16^{\mathrm{L}}, 19,103,116,139,155,182,217$, No. 948

20,7

20,8

20,9

20,10

20,11

20,12

20,13 $1,4,5,9,10,14^{\mathrm{L}}, 15^{\mathrm{L}}, 19, * 82,103,116,139,168,182,217,335^{\mathrm{L}}, 369,649$ $1,4,5,9,10,14^{\mathrm{L}}, 15^{\mathrm{L}}, 19, * 82,103,121,127,139,168,182,217,335^{\mathrm{L}}, 649$

20,14 $1,4,5,9,10,14^{\mathrm{L}}, 15^{\mathrm{L}}, 19,103,121,127,139,168,182, * 217$ $1,4,5,9,10,14^{\mathrm{L}}, 15^{\mathrm{L}}, 19,103, * 127,139,168,182,335^{\mathrm{L}}$ $1,4,5,7,9,10,14^{\mathrm{L}}, 15^{\mathrm{L}}, 19,103,143,168,182,335^{\mathrm{L}}, 339^{\mathrm{L}}$ $1,4,5,7,9,10,14^{\mathrm{L}}, 15^{\mathrm{L}}, 19,103,143, * 168,182,335^{\mathrm{L}}, 339^{\mathrm{L}}$ $1,4,5,7,9,10,14^{\mathrm{L}}, 15^{\mathrm{L}}, 19, * 82,103,143,168,182,339^{\mathrm{L}}$ $1,4,5,7,9,10,14^{\mathrm{L}}, 15^{\mathrm{L}}, 19,82,103,116,143,168,182,339^{\mathrm{L}}$ $1,4,5,9,10,14^{\mathrm{L}}, 15^{\mathrm{L}}, 19,103,116,127,139,168,182,335^{\mathrm{L}}, 339^{\mathrm{L}}, 369$

20,15

20,16 $1,4,5,7,9,10,14^{\mathrm{L}}, 15^{\mathrm{L}}, 19,103,116,127,168,182,335^{\mathrm{L}}, 339^{\mathrm{L}}, 369$ $1,4,5,7,9,10,14^{\mathrm{L}}, 15^{\mathrm{L}}, 19,103,116,127,168,182,339^{\mathrm{L}}, 369$ $1,4,5,7,9,10,14^{\mathrm{L}}, 15^{\mathrm{L}}, 19,103,115,116,168,182,308^{\mathrm{L}}, 335^{\mathrm{L}}, 339^{\mathrm{L}}, 369$ $1,4,5,9,10,14^{\mathrm{L}}, 15^{\mathrm{L}}, 19,103,115,116,168,182,268,308^{\mathrm{L}}, 335^{\mathrm{L}}, 339^{\mathrm{L}}, 369$ 
$20,17 \quad 1,4,5,9,10,14^{\mathrm{L}}, 15^{\mathrm{L}}, 19,103,115,116,143,168,182,268,335^{\mathrm{L}}, 339^{\mathrm{L}}, 369$

$20,18 \quad 1,4,5,9,10,14^{\mathrm{L}}, 15^{\mathrm{L}}, 19,103,115,116,168,182,268,335^{\mathrm{L}}, 339^{\mathrm{L}}, 369$

$20,191,4,5,9,10,15^{\mathrm{L}}, 19,66,103,115,116,168,182,268,369,663^{\mathrm{L}}$ (nur Incipit, Typikon)

$20,20 \quad 1,4,5,9,10,15^{\mathrm{L}}, 19,66,82,103,115,116,168,182,268,369$

$20,21 \quad 1,4,5,9,10,15^{\mathrm{L}}, 19,66,82,103,115,116,168,182,268,369$

$20,221,4,5,9,10,15^{\mathrm{L}}, 19,66,103,115,116,168,182,268,302^{\mathrm{L}}, 369$

$20,23 \quad 1,4,5,9,15^{\mathrm{L}}, 19,66,103,115,116,168,182,268,302^{\mathrm{L}}, 369$

$20,241,4,5,9,10,14^{\mathrm{L}}, 15^{\mathrm{L}}, 19,66,103,115,116,143,168,182,268,335^{\mathrm{L}}, 342^{\mathrm{L}}, 369$

$20,251,4,5,9,10,14^{\mathrm{L}}, 15^{\mathrm{L}}, 19,66,82,103,115,116,143,168,182,256,268,271,335^{\mathrm{L}}, 340^{\mathrm{L}}$, $342^{\mathrm{L}}, 369$

$20,261,4,5,9,10,14^{\mathrm{L}}, 15^{\mathrm{L}}, 19,66,82,103,106,115,116,168,182,256,268,271,335^{\mathrm{L}}, 340^{\mathrm{L}}$, $342^{\mathrm{L}}, 369$

$20,27 \quad 1,4,5,9,10,14^{\mathrm{L}}, 15^{\mathrm{L}}, 19,66,103,106,115,116,168,182,335^{\mathrm{L}}, 342^{\mathrm{L}}$

$20,281,4,5,9,10,14^{\mathrm{L}}, 15^{\mathrm{L}}, 19,66,103,106,115,116,168,182,335^{\mathrm{L}}, 336^{\mathrm{L}}, 342^{\mathrm{L}}$

$20,291,4,5,9,10,14^{\mathrm{L}}, 15^{\mathrm{L}}, 19,66,103,106,115,116,143,168,182,335^{\mathrm{L}}, 336^{\mathrm{L}}, 340^{\mathrm{L}}, 342^{\mathrm{L}}$

$20,301,4,5,9,10,14^{\mathrm{L}}, 15^{\mathrm{L}}, 19,66,82,103,106,115,116,143,182,335^{\mathrm{L}}, 336^{\mathrm{L}}, 340^{\mathrm{L}}, 342^{\mathrm{L}}$

$20,311,4,5,9,10,14^{\mathrm{L}}, 15^{\mathrm{L}}, 19,66,82,106,115,116,136,143,182,271,335^{\mathrm{L}}, 336^{\mathrm{L}}, 342^{\mathrm{L}}, 369$

$21,1 \quad 1,4,5,9,10,19,115,116,182,271,293^{\mathrm{L}}, 370$

$21,2 \quad 1,4,5,9,10,19,106,110,115,116,182,271,293^{\mathrm{L}}, 370$

$21,3 \quad 1,4,5,9,10,19,82,110,116,136,182,293^{\mathrm{L}}, 370$

$21,4 \quad 1,4,5,9,10,19,82,106,110,116,136,182,293^{\mathrm{L}}, 370$

$21,5 \quad 1,4,5,9,10,19,106,110,116,136,182,293^{\mathrm{L}}, 370$

$21,6 \quad 1,4,5,9,10,19,106,110,116,136,182,271,293^{\mathrm{L}}, 370$

$21,7 \quad 1,4,5,9,10,19,106,110,116,136,163,182,271,293^{\mathrm{L}}, 317^{\mathrm{L}}, 370$

$21,8 \quad 1,4,5,9,10,19,110,116,136,163,182,293^{\mathrm{L}}, 317^{\mathrm{L}}, 370$

$21,9 \quad 1,4,5,9,10,19,110,116,136,163,182,256,293^{\mathrm{L}}, 317^{\mathrm{L}}$

$21,101,4,5,9,19,110,116,136,163,182,256,293^{\mathrm{L}}, 317^{\mathrm{L}}$

$21,11 \quad 1,4,5,9,10,19,110,116,136,163,182,256,293^{\mathrm{L}}, 317^{\mathrm{L}}$

$21,121,4,5,9,10,19,110,116,136,163,182,271,317^{\mathrm{L}}, 663^{\mathrm{L}}$ (nur Incipit, Typikon)

$21,13 \quad 1,4,5,9,10,19,110,116,131,136,163,182,271$

$21,14 \quad 1,4,5,9,10,19,110,116,131,136,163,182,255$

$21,15 \quad 1,4,5,9,10,19,116,131,136,163,182,255,293^{\mathrm{L}}$

$21,16 \quad 1,4,5,9,10,19,22,103,116,131,136,163,182,293^{\mathrm{L}}$

$21,17 \quad 1,4,5,9,10,19,22,103,116,131,163,182,293^{\mathrm{L}}$

$21,18 \quad 1,4,5,9,10,19,93,103,131,163,182,237,255,293^{\mathrm{L}}$

$21,19 \quad 1,4,5,9,10,19,103,131,163,182,237,293^{\mathrm{L}}$

$21,20 \quad 1,4,5,9,19,65, * 82,103,131,163,182,237,293^{\mathrm{L}}$

$21,21 \quad 1,4,5,9,10,19,65,82,93,103,131,163,182,237$

$21,22 \quad 1,4,5,9,10,19,65,82,93,103,131,163,182,237$

$21,23 \quad 1,4,5,9,10,19,65, * 82,103,131,163,182,237$

$21,24 \quad 1,4,5,9,19,65,103,131,163,237$

$21,251,4,5,9,10,19,65,82,103,131,163,237,393^{\mathrm{var}}$

Subscriptio

$1,4,5,9,65,82,103,131,163,237,408^{\mathrm{L}}$ (scriptio superior) 
Glossen, liturgische Verweise und Titel

pbo, 14 ${ }^{\mathrm{L}}, 15^{\mathrm{L}}, 16^{\mathrm{L}}, 59^{\mathrm{L}}, 89,103$ (Glossen, Gebet, liturgische Verweise und griechisch $\left.20,1-7^{\mathrm{lac}}\right), 110,115,116,126,127,140,154,234,291^{\mathrm{L}}, 293^{\mathrm{L}}, 294^{\mathrm{L}}, 295^{\mathrm{L}}, 297^{\mathrm{L}}, 298^{\mathrm{L}}, 299^{\mathrm{L}}$ (liturgische Rubrik, siehe Anhang 2 - 1.4. Übersicht zu Glossen, liturgischen Vermerken und Überschriften), 300 ${ }^{\mathrm{L}}, 301^{\mathrm{L}}, 302^{\mathrm{L}}, 305^{\mathrm{L}}, 306^{\mathrm{L}}, 313^{\mathrm{L}}, 324^{\mathrm{L}}, 335^{\mathrm{L}}, 336^{\mathrm{L}}, 337^{\mathrm{L}}$, $339^{\mathrm{L}}, 340^{\mathrm{L}}, 342^{\mathrm{L}}, 347^{\mathrm{L}}, 349^{\mathrm{L}}, 352,396^{\mathrm{L}}, 402$ (Hermenien), 408 ${ }^{\mathrm{L}}, 411^{\mathrm{L}}, 440^{\mathrm{L}}, 452^{\text {var. }}, 663^{\mathrm{L}}$, No. 948

Eusebianische Kanonzahlen

131, 285

Kephalaia, griechisch

9, $\{47$ (Nummern am Text nicht erkennbar) $\}, 124,131,142,197,285,291^{\mathrm{L}}$

Titel

9, 47, 124 (zugleich Titel des griechischen Kephalaions), 131, 197

Kephalaia, koptisch (Abschnittsnummern)

$10,12,85,108,109,110,115,116,124,125,134,139,155,162,167,174,181,182,250$, $260,285,286,291^{\mathrm{L}}, 292^{\mathrm{L}}, 293^{\mathrm{L}}, 369,396^{\mathrm{L}}, 663^{\mathrm{L}}$

Titel

110, 124 (zugleich Titel des griechischen Kephalaions), 126, \{234 (unsicher) $\}$

Makulaturstücke mit Schrift

12 (dokumentarische Texte), 102 (Schrift nicht sicher zu deuten), 103 (literarische

Stichoi-Angabe

Texte), 124 (literarischer Text), 126 (literarischer Text)

131

Kolophone

4, 5, 10, 19 (drei, einer arabisch), 65, 82, 349 (arabisch, nicht behandelt), 408 ${ }^{\mathrm{L}}$ (scriptio superior) 